\title{
Re-exploring Molecular Complexity with ALMA (ReMoCA): interstellar detection of urea
}

\author{
A. Belloche ${ }^{1}$, R. T. Garrod ${ }^{2}$, H. S. P. Müller ${ }^{3}$, K. M. Menten ${ }^{1}$, I. Medvedev ${ }^{4}$, J. Thomas ${ }^{4}$, and Z. Kisiel ${ }^{5}$ \\ ${ }^{1}$ Max-Planck-Institut für Radioastronomie, Auf dem Hügel 69, 53121 Bonn, Germany \\ e-mail: belloche@mpi fr-bonn.mpg.de \\ 2 Departments of Chemistry and Astronomy, University of Virginia, Charlottesville, VA 22904, USA \\ ${ }^{3}$ I. Physikalisches Institut, Universität zu Köln, Zülpicher Str. 77, 50937 Köln, Germany \\ ${ }^{4}$ Department of Physics, Wright State University, Dayton, OH 45435, USA \\ ${ }^{5}$ Institute of Physics, Polish Academy of Sciences, Al. Lotnikow 32/46, 02-668 Warszawa, Poland
}

Received 7 March 2019 / Accepted 4 June 2019

\begin{abstract}
Context. Urea, $\mathrm{NH}_{2} \mathrm{C}(\mathrm{O}) \mathrm{NH}_{2}$, is a molecule of great importance in organic chemistry and biology. Two searches for urea in the interstellar medium have been reported in the past, but neither were conclusive.

Aims. We want to take advantage of the increased sensitivity and angular resolution provided by the Atacama Large Millimeter/submillimeter Array (ALMA) to search for urea toward the hot molecular cores embedded in the high-mass-star-forming region Sgr B2(N).

Methods. We used the new spectral line survey named ReMoCA (Re-exploring Molecular Complexity with ALMA) that was performed toward Sgr B2(N) with ALMA in its observing cycle 4 between 84 and $114 \mathrm{GHz}$. The spectra were analyzed under the local thermodynamic equilibrium approximation. We constructed a full synthetic spectrum that includes all the molecules identified so far. We used new spectroscopic predictions for urea in its vibrational ground state and first vibrationally excited state to search for this complex organic molecule in the ReMoCA data set. We employed the gas-grain chemical kinetics model MAGICKAL to interpret the astronomical observations.

Results. We report the secure detection of urea toward the hot core Sgr B2(N1) at a position called N1S slightly offset from the continuum peak, which avoids obscuration by the dust. The identification of urea relies on nine clearly detected transitions. We derive a column density of $2.7 \times 10^{16} \mathrm{~cm}^{-2}$ for urea, two orders of magnitude lower than the column density of formamide, and one order of magnitude below that of methyl isocyanate, acetamide, and N-methylformamide. The latter molecule is reliably identified toward N1S with 60 clearly detected lines, confirming an earlier claim of its tentative interstellar detection. We report the first interstellar detections of $\mathrm{NH}_{2} \mathrm{CH}^{18} \mathrm{O}$ and ${ }^{15} \mathrm{NH}_{2} \mathrm{CHO}$. We also report the nondetection of urea toward the secondary hot core Sgr B2(N2) with an abundance relative to the other four species at least one order of magnitude lower than toward the main hot core. Our chemical model roughly reproduces the relative abundances of formamide, methyl isocyanate, acetamide, and $\mathrm{N}$-methylformamide, but it overproduces urea by at least one order of magnitude.

Conclusions. Urea is clearly detected in one of the hot cores. Comparing the full chemical composition of Sgr B2(N1S) and Sgr B2(N2) may help understand why urea is at least one order of magnitude less abundant in the latter source.
\end{abstract}

Key words. astrochemistry - line: identification - radio lines: ISM - ISM: molecules - ISM: individual objects: Sagittarius B2

\section{Introduction}

The chemical composition of astronomical sources, in particular star-forming regions, can be a good probe of their physical properties, their evolutionary stage, and their history. This knowledge can even help us establish connections with the chemical composition of small bodies like comets and asteroids in our solar system, and thereby possibly learn about the conditions that were prevailing when life appeared on Earth. With the advent of broadband receivers at observational facilities, spectral line surveys covering wide frequency ranges with sufficient spectral resolution have become steadily more efficient and turned into ideal tools to derive the chemical composition of (many) astronomical sources in a systematic way (e.g., Bergner et al. 2017; Bonfand et al. 2017). In particular, such surveys are essential for the robust identification of complex organic molecules (COMs) in the interstellar medium (ISM), defined as molecules containing carbon with six atoms or more (Herbst \& van Dishoeck
2009). More than 200 interstellar molecules have been identified in the ISM since the advent of radio astronomy (e.g., McGuire 2018), with an approximately constant detection rate of about four new molecules every year since the early 1970s. While new molecules, including ions and radicals, with few atoms are still being discovered, much effort has been put into searching for more complex species with the aim of exploring the degree of complexity that interstellar chemistry can achieve and understanding the chemical processes that lead to this complexity.

Complex organic molecules have been found in diverse types of astronomical environments, but the first detections of these species were most of the time achieved in star-forming regions. The hot $(\sim 100-200 \mathrm{~K})$ and dense $\left(10^{6}-10^{8} \mathrm{~cm}^{-3}\right)$ molecular regions around new born (proto)stars, called hot cores and hot corinos around high-mass and low-mass objects, respectively, have indeed turned out to be excellent targets to probe the complexity of interstellar chemistry. Among these sources, the hot 
cores embedded in the giant molecular cloud Sagittarius B2 (Sgr B2) close to the Galactic center, despite their large distance from us ( $8.3 \mathrm{kpc}$, Reid et al. 2014), are prime targets because their high column densities $\left(10^{24}-10^{25} \mathrm{~cm}^{-2}\right.$ at arcsecond scale, Qin et al. 2011; Bonfand et al. 2017) facilitate the detection of low-abundance molecules.

Building on a spectral line survey performed with the $30 \mathrm{~m}$ single-dish telescope of the Institut de Radioastronomie Millimétrique (hereafter IRAM $30 \mathrm{~m}$ telescope) toward Sgr B2(N) and Sgr B2(M) (Belloche et al. 2013), which led to the detection of several new COMs (Belloche et al. 2008, 2009), we have taken advantage of the advent of the Atacama Large Millimeter/submillimeter Array (ALMA) to perform the EMoCA survey (Exploring Molecular Complexity with ALMA) toward $\mathrm{Sgr} \mathrm{B}(\mathrm{N})$ in order to probe further the chemical composition of the hot cores embedded within it (Belloche et al. 2016). The high angular resolution achieved with ALMA for this survey was essential to make significant progress given that the single-dish spectra were already close to the spectral confusion limit. With a half-power beam width (HPBW) of $\sim 1.6^{\prime \prime}$, the EMoCA survey revealed that the rotational lines detected toward the northern hot core of Sgr B2(N), which we call Sgr B2(N2), had widths as narrow as $\sim 5 \mathrm{~km} \mathrm{~s}^{-1}$, whereas the main hot core, Sgr B2(N1), located $\sim 5^{\prime \prime}$ to the south of Sgr B2(N2), still had broader linewidths $\left(\sim 7 \mathrm{~km} \mathrm{~s}^{-1}\right)$ and showed many lines with prominent wings. While the resulting spectral confusion prevented any significant progress in determining the chemical composition of Sgr $\mathrm{B}(\mathrm{N} 1)$ compared to what we achieved with the single-dish telescope, the narrow linewidths of Sgr B2(N2) and its separation from Sgr B2(N1) allowed us to identify two new COMs toward Sgr B2(N2) in the EMoCA survey: the branched alkyl molecule iso-propyl cyanide $\left(i-\mathrm{C}_{3} \mathrm{H}_{7} \mathrm{CN}\right.$, Belloche et al. 2014) and, tentatively, $\mathrm{N}$-methylformamide $\left(\mathrm{CH}_{3} \mathrm{NHCHO}\right.$, Belloche et al. 2017). To make further progress on the chemical composition of Sgr B2(N1), we performed a new spectral line survey with ALMA at even higher angular resolution and sensitivity. In keeping with the EMoCA survey, we call this new survey ReMoCA, which stands for Re-exploring Molecular Complexity with ALMA. As an initial outcome of ReMoCA, we present the results of our search for urea toward Sgr B2.

Urea $\left(\mathrm{NH}_{2} \mathrm{C}(\mathrm{O}) \mathrm{NH}_{2}\right)$, also called carbonyl diamide or carbamide, is a complex organic molecule in the astrochemical sense. It assumes a special role in the history of modern science. In 1828, Friedrich Wöhler synthesized this organic molecule, which is associated with biological sources, out of inorganic compounds (Wöhler 1828). This is widely seen as the start of modern organic chemistry, effectively rendering obsolete the need for a special vital force associated with living organisms only, which was the mainstream thinking at that time. Urea is thermodynamically speaking the most stable molecule in the $\mathrm{CH}_{4} \mathrm{~N}_{2} \mathrm{O}$ family (Fourré et al. 2016). None of the $\mathrm{CH}_{4} \mathrm{~N}_{2} \mathrm{O}$ isomers have been securely detected in the ISM so far, but urea has been identified in meteorites (e.g., Hayatsu et al. 1975) and laboratory experiments conducted on interstellar ice analogs have shown that urea can be formed under ultraviolet irradiation (e.g., Nuevo et al. 2010). Both results suggest that urea may exist in the ISM and motivate astronomers to search for it. Two searches for urea have been reported in the literature so far. Raunier et al. (2004) compared the infrared spectra of the young stellar object NGC 7538 IRS9 recorded with the Short Wavelength Spectrometer (SWS) onboard the Infrared Space Observatory (ISO) to their laboratory spectra of ices containing ammonia, water, and the products of the ultraviolet irradiation of isocyanic acid that leads to the formation of urea, among other species. The similarity between the shapes of the broad bands detected with ISO and the laboratory spectra led them to claim a tentative identification of urea (and formamide) in NGC 7538 IRS9. Because of the nonuniqueness of IR band assignments, the astrochemical community has not retained this tentative identification.

More recently, Remijan et al. (2014) reported on a multitelescope campaign performed to search for urea in the millimeter-wavelength range. The team targeted Sgr B2(N) with five observational facilities, both interferometers and singledish telescopes. They covered urea transitions between 100 and $250 \mathrm{GHz}$ and found possible evidence for transitions of this molecule at 102 and $232 \mathrm{GHz}$ with four spectral lines assigned to urea using a local-thermodynamic-equilibrium (LTE) synthetic spectrum of its rotational emission. Other transitions predicted by their model were consistent with the observed spectra but could not be assigned due to blends with other species. Due to the small number of assigned transitions and the complexity of the hot-core spectrum, which they did not attempt to model in its entirety, Remijan et al. (2014) refrained from claiming an interstellar detection of urea.

We report below a detection of urea with ALMA using the ReMoCA survey. Section 2 presents the observational details and explains how the ALMA data were reduced. Section 3 describes how the spectroscopic predictions of urea were obtained from laboratory measurements. Section 4 reports our astronomical results on urea as well as several other related molecules, in particular N-methylformamide. In Sect. 5, we put the astronomical results into a broader astrochemical context using numerical simulations of interstellar chemistry. The results are discussed in Sect. 6 and we give the conclusion of our findings in Sect. 7.

\section{Observations and data reduction}

\subsection{Observations}

The observations of the ReMoCA survey were conducted in a very similar way as for the EMoCA survey (Belloche et al. 2016). We performed a complete spectral line survey toward Sgr B2(N) with ALMA between 84.1 and $114.4 \mathrm{GHz}$. The phase center was set at $(\alpha, \delta)_{\mathrm{J} 2000}=\left(17^{\mathrm{h}} 47^{\mathrm{m}} 19^{\mathrm{s}} .87,-28^{\circ} 22^{\prime} 16^{\prime \prime} \cdot 0\right)$, a position that is located halfway between the hot cores Sgr B2(N1) and (N2) that are separated by $4.9^{\prime \prime}$ in the north-south direction (Belloche et al. 2016). The size (HPBW) of the primary beam of the $12 \mathrm{~m}$ antennas varies between $69^{\prime \prime}$ at $84 \mathrm{GHz}$ and $51^{\prime \prime}$ at $114 \mathrm{GHz}$ (Remijan et al. 2015).

Details about the observational setup are given in Table 1. The survey was divided into five spectral setups that were observed independently from each other. Each setup was observed in four observing blocks of 47-50 min on-source integration time each. Each setup was observed in only one polarization and delivered four spectral windows of bandwidths $1875 \mathrm{MHz}$ each, two per sideband. The sideband separation is $12 \mathrm{GHz}$. Each pair of adjacent spectral windows has an overlap of about $50 \mathrm{MHz}$. The observations were performed with a channel spacing of $244.141 \mathrm{kHz}$ and the spectra were smoothed to a resolution of $488.3 \mathrm{kHz}\left(1.7-1.3 \mathrm{~km} \mathrm{~s}^{-1}\right)$. All observations were performed during ALMA's observing cycle 4.

\subsection{Data reduction}

The data was calibrated and imaged with the Common Astronomy Software Applications package (CASA), version 4.2.0 (r28322) for setups S1 to S4 and version 4.2.1 (r29047) for setup S5. We used the procedures provided by the Joint ALMA Observatory to apply the bandpass, amplitude, and phase 
Table 1. Observational setups of the ReMoCA survey.

\begin{tabular}{|c|c|c|c|c|c|c|c|c|c|c|c|}
\hline \multirow[t]{2}{*}{ Setup } & \multicolumn{2}{|c|}{ Frequency range } & \multirow{2}{*}{$\begin{array}{c}\text { Date of } \\
\text { observation } \\
\text { yyyy-mm-dd }\end{array}$} & \multirow{2}{*}{$\begin{array}{l}t_{\text {start }}(a) \\
\text { (UTC) } \\
\text { hh:mm }\end{array}$} & \multirow[t]{2}{*}{$N_{\mathrm{a}}{ }^{(b)}$} & \multirow{2}{*}{$\begin{array}{l}\text { Baseline } \\
\text { range }(c) \\
(\mathrm{m})\end{array}$} & \multirow{2}{*}{$\begin{array}{l}t_{\text {int }}(d) \\
(\min )\end{array}$} & \multirow{2}{*}{$\begin{array}{l}\operatorname{pwv}^{(e)} \\
(\mathrm{mm}) \\
\end{array}$} & \multicolumn{3}{|c|}{ Calibrators $(f)$} \\
\hline & $\begin{array}{c}\text { LSB } \\
(\mathrm{GHz})\end{array}$ & $\begin{array}{c}\text { USB } \\
\text { (GHz) }\end{array}$ & & & & & & & $\mathrm{B}$ & A & $\mathrm{P}$ \\
\hline \multirow[t]{4}{*}{$\mathrm{S} 1$} & $84.1-87.8$ & $96.1-99.8$ & $2016-10-29$ & $22: 19$ & 40 & 19-1100 & 47 & 2.3 & 1 & 1 & 2 \\
\hline & & & $2016-10-30$ & $20: 33$ & 42 & $17-1100$ & 47 & 1.2 & 1 & 3 & 2 \\
\hline & & & 2016-11-03 & $20: 10$ & 42 & $19-1100$ & 47 & 1.4 & 1 & 3 & 2 \\
\hline & & & 2016-11-04 & $20: 11$ & 40 & $21-1100$ & 47 & 2.0 & 1 & 3 & 2 \\
\hline \multirow[t]{4}{*}{$\mathrm{S} 2$} & $87.8-91.5$ & $99.8-103.5$ & 2016-11-04 & $21: 39$ & 40 & $21-1100$ & 47 & 2.2 & 1 & 3 & 2 \\
\hline & & & 2016-11-06 & $15: 52$ & 40 & $19-1100$ & 47 & 2.0 & 4 & 3 & 2 \\
\hline & & & $2017-05-10$ & $06: 14$ & 43 & $17-1100$ & 47 & 2.7 & 1 & 1 & 2 \\
\hline & & & $2017-07-01$ & $06: 05$ & 36 & $21-2200$ & 47 & 1.0 & 1 & 3 & 2 \\
\hline \multirow[t]{4}{*}{$\mathrm{S} 3$} & 91.4-95.1 & $103.4-107.1$ & $2017-05-22$ & $04: 50$ & 43 & $15-1100$ & 47 & 2.8 & 1 & 3 & 2 \\
\hline & & & $2017-05-22$ & $06: 12$ & 43 & $15-1100$ & 47 & 4.0 & 1 & 1 & 2 \\
\hline & & & $2017-05-22$ & $07: 23$ & 43 & $15-1100$ & 47 & 3.6 & 1 & 3 & 2 \\
\hline & & & $2017-05-22$ & $08: 40$ & 43 & $15-1100$ & 47 & 3.8 & 1 & 3 & 2 \\
\hline \multirow[t]{4}{*}{$\mathrm{S} 4$} & $95.1-98.8$ & $107.1-110.8$ & $2017-07-15$ & $01: 25$ & 42 & $19-1500$ & 48 & 0.8 & 1 & 3 & 2 \\
\hline & & & $2017-07-23$ & $01: 40$ & 46 & $17-3600$ & 48 & 3.0 & 1 & 3 & 2 \\
\hline & & & 2017-07-23 & $02: 59$ & 46 & $17-3600$ & 48 & 2.8 & 1 & 3 & 2 \\
\hline & & & $2017-07-23$ & 04:18 & 46 & $17-3600$ & 48 & 2.1 & 1 & 3 & 2 \\
\hline \multirow[t]{4}{*}{ S5 } & $98.7-102.4$ & $110.7-114.4$ & $2017-07-23$ & $05: 36$ & 46 & $17-3600$ & 50 & 1.6 & 1 & 1 & 2 \\
\hline & & & $2017-07-24$ & $02: 27$ & 44 & $31-3600$ & 50 & 0.5 & 1 & 3 & 2 \\
\hline & & & 2017-07-24 & $03: 47$ & 44 & $31-3600$ & 50 & 0.3 & 1 & 3 & 2 \\
\hline & & & $2017-07-24$ & 05:06 & 44 & $31-3600$ & 50 & 0.3 & 1 & 1 & 2 \\
\hline
\end{tabular}

Notes. ${ }^{(a)}$ Start time of observation. ${ }^{(b)}$ Number of ALMA $12 \mathrm{~m}$ antennas. ${ }^{(c)}$ Minimum and maximum projected baseline separations. ${ }^{(d)}$ On-source integration time. ${ }^{(e)}$ Precipitable water vapour content of the atmosphere. ${ }^{(f)}$ Bandpass $(\mathrm{B})$, amplitude (A), and phase $(\mathrm{P})$ calibrators. The calibrators are 1: J1924-2914, 2: J1744-3116, 3: J1733-1304, 4: J1517-2422.

calibrations. In addition, three or four iterations of selfcalibration were performed using a strong spectral line detected toward Sgr B2(N1) in each setup. The deconvolution was performed with the csclean imager mode and a Briggs weighting scheme with a robust parameter of 0.5 . The pixel size was set to $0.12^{\prime \prime}$ for setups $\mathrm{S} 1-\mathrm{S} 3$ and $0.06^{\prime \prime}$ for setups S4-S5, with $1000 \times$ 1000 pixels and $1890 \times 1890$ pixels, respectively.

Given the high number of spectral lines detected toward the hot cores, their different systemic velocities, and the velocity gradients across some of them, it is impossible to split the line and continuum emission in the Fourier domain. Therefore, we performed the splitting in the image plane, in an automatic way using the GILDAS/CLASS software ${ }^{1}$. Because of the presence of numerous absorption lines produced by the Sgr B2 molecular cloud itself and the diffuse and translucent clouds along the line of sight (see, e.g., Thiel et al. 2019), selecting the channels suitable for fitting the level of the continuum emission in an automatic way toward the hot core positions is not straightforward. We selected four positions that are free of hot-core emission but are associated with a strong continuum emission, among others the ultra-compact $\mathrm{H}$ II (UCH II) regions $\mathrm{K} 4$ and $\mathrm{K} 1$, in order to find the channels that contain signal in absorption (and some in emission as well, coming from larger scales). Then, for each pixel, we compute the channel intensity distribution after masking these channels. Provided all channels with absorption have been properly masked and the baseline does not contain any artifacts due to inaccuracies of the bandpass calibration or the imaging process, we expect the part of the channel distribution below the continuum baseline to follow a Gaussian pattern. We estimated the width of this lower part on pixels that appeared

1 See http://www.iram.fr/IRAMFR/GILDAS free from line emission and used this width to define the level of the baseline in each pixel. The beam sizes and position angles as well as the median noise levels measured in the channel maps of the continuum-subtracted datacubes are reported in Table 2. The beam sizes are 2.5-4 times smaller than the ones achieved with the EMoCA survey. The sensitivity in flux density of the ReMoCA survey is a factor of three times better than that of the EMoCA survey.

In practice, the spectral baselines in some areas of the field of view suffer from artifacts that we have not yet fully understood and that may be due to inaccuracies of the bandpass calibration or to missing information related to the incomplete sampling of the UV plane. Therefore, the splitting of the line and continuum emission performed so far is still preliminary and we plan to improve it in the future. Nevertheless, it is sufficient to start analyzing the continuum-subtracted spectra of the hot cores detected in the field of view.

\section{Spectroscopy}

The rotational spectrum of urea has received only modest attention in the spectroscopic community. Until recently the investigations were limited to frequencies below $50 \mathrm{GHz}$ and to the ground vibrational state. Brown et al. (1975) studied the microwave spectrum and determined its sizeable dipole moment $\mu=\mu_{b}=3.83 \mathrm{D}$ as well as ${ }^{14} \mathrm{~N}$ quadrupole coupling parameters. Kasten \& Dreizler (1986) and Kretschmer et al. (1996) studied the ${ }^{14} \mathrm{~N}$ hyperfine structure (HFS) in greater detail and determined unsplit rest frequencies with greater accuracy. Godfrey et al. (1997) studied the rotational spectra of several isotopologs to determine the structure of urea, in particular the exact shape of the molecule. They also mentioned the identification of 
Table 2. Beam sizes and noise levels.

\begin{tabular}{|c|c|c|c|c|c|c|}
\hline \multicolumn{2}{|c|}{$\mathrm{S} .{ }^{(a)} \mathrm{W} .{ }^{(b)}$} & \multirow{2}{*}{$\begin{array}{c}\text { Freq. range }{ }^{(c)} \\
(\mathrm{MHz})\end{array}$} & \multicolumn{2}{|c|}{ Synthesized beam } & \multicolumn{2}{|c|}{$\mathrm{rms}^{(e)}$} \\
\hline & & & $\begin{array}{c}H P B W \\
\left({ }^{\prime \prime} \times{ }^{\prime \prime}\right) \\
\end{array}$ & $\begin{array}{c}\mathrm{PA}^{(d)} \\
\left(^{\circ}\right)\end{array}$ & $\begin{array}{c}(\mathrm{mJy} \\
\left.\text { beam }^{-1}\right)\end{array}$ & $(\mathrm{K})$ \\
\hline \multirow[t]{4}{*}{$\mathrm{S} 1$} & 0 & $84112-85990$ & $0.86 \times 0.67$ & -84 & 0.98 & 0.29 \\
\hline & 1 & $85938-87815$ & $0.90 \times 0.66$ & -87 & 0.94 & 0.26 \\
\hline & 2 & $96116-97993$ & $0.74 \times 0.59$ & -84 & 0.92 & 0.27 \\
\hline & 3 & $97941-99818$ & $0.72 \times 0.57$ & -86 & 0.94 & 0.29 \\
\hline \multirow[t]{4}{*}{$\mathrm{S} 2$} & 0 & $87763-89640$ & $0.85 \times 0.62$ & 87 & 1.02 & 0.30 \\
\hline & 1 & $89588-91465$ & $0.93 \times 0.61$ & 83 & 1.05 & 0.28 \\
\hline & 2 & $99766-101643$ & $0.72 \times 0.60$ & 83 & 0.82 & 0.23 \\
\hline & 3 & $101591-103468$ & $0.72 \times 0.62$ & 66 & 0.74 & 0.19 \\
\hline \multirow[t]{4}{*}{ S3 } & 0 & $91403-93280$ & $0.70 \times 0.62$ & 89 & 0.85 & 0.28 \\
\hline & 1 & $93228-95105$ & $0.69 \times 0.59$ & -86 & 0.81 & 0.27 \\
\hline & 2 & $103405-105282$ & $0.63 \times 0.53$ & -85 & 0.87 & 0.29 \\
\hline & 3 & $105230-107107$ & $0.61 \times 0.52$ & -86 & 0.92 & 0.31 \\
\hline \multirow[t]{4}{*}{$\mathrm{S} 4$} & 0 & $95062-96939$ & $0.57 \times 0.46$ & -53 & 0.39 & 0.20 \\
\hline & 1 & $96887-98764$ & $0.56 \times 0.45$ & -53 & 0.39 & 0.20 \\
\hline & 2 & $107064-108942$ & $0.51 \times 0.41$ & -54 & 0.43 & 0.22 \\
\hline & 3 & $108890-110767$ & $0.50 \times 0.40$ & -54 & 0.45 & 0.23 \\
\hline \multirow[t]{4}{*}{ S5 } & 0 & $98714-100591$ & $0.43 \times 0.30$ & -78 & 0.69 & 0.66 \\
\hline & 1 & $100539-102417$ & $0.42 \times 0.29$ & -78 & 0.68 & 0.66 \\
\hline & 2 & $110717-112594$ & $0.39 \times 0.26$ & -77 & 0.81 & 0.78 \\
\hline & 3 & $112542-114419$ & $0.38 \times 0.25$ & -77 & 0.95 & 0.95 \\
\hline
\end{tabular}

Notes. ${ }^{(a)}$ Setup. ${ }^{(b)}$ Spectral window. ${ }^{(c)}$ The frequencies correspond to rest frequencies at a systemic velocity of $62 \mathrm{~km} \mathrm{~s}^{-1}$. ${ }^{(d)}$ Position angle East from North. ${ }^{(e)}$ Median rms noise level measured in the channel maps of the continuum-removed data cubes.

transitions pertaining to two excited vibrational states whose vibrational energy they estimated from relative intensities to be $\sim 155 \mathrm{~cm}^{-1}(\sim 225 \mathrm{~K})$ and $\sim 365 \mathrm{~cm}^{-1}(\sim 525 \mathrm{~K})$, which they interpreted as the $\mathrm{NH}_{2}$ wagging fundamental and first overtone states, respectively. Additional investigations of the isotopologs containing one and two ${ }^{15} \mathrm{~N}$ were also carried out (Kassi et al. 2004).

Remijan et al. (2014) reported fairly extensive transition rest frequencies in the range of 59 to $233 \mathrm{GHz}$ in their account of searching for urea toward Sgr B2(N). At about the same time, we started an investigation into the millimeter and submillimeter spectrum of urea (Thomas et al. 2013, 2014; Kisiel et al. 2014). Our laboratory spectra of urea were collected with a system reported in Fosnight et al. (2013). The sample was thermally evolved into vapor by heating solid urea to $120^{\circ} \mathrm{C}$. A steady flow of urea through a two-meter long absorption cell heated to $120^{\circ} \mathrm{C}$ facilitated the acquisition of continuous spectra in the $210-270$ and $300-500 \mathrm{GHz}$ bands. Two commercial heterodyne systems produced by Virginia Diodes, Inc. ${ }^{2}$ covering these two bands were used in the study. The heterodyne systems were driven by a custom microwave synthesizer reported in Medvedev et al. (2010). The spectra were recorded using a frequency modulation technique. The second-derivative (2f) spectra were digitized from the output of a lock-in amplifier. The $2 \mathrm{f}$ spectra were then normalized by the baseline power, recorded independently.

Besides transitions within the ground vibrational state of urea, we identified numerous transitions within excited vibrational states of which three have been analyzed thus far along with the ground vibrational state. Over one thousand transitions

\footnotetext{
2 Available from http://www.virginiadiodes.com/
}

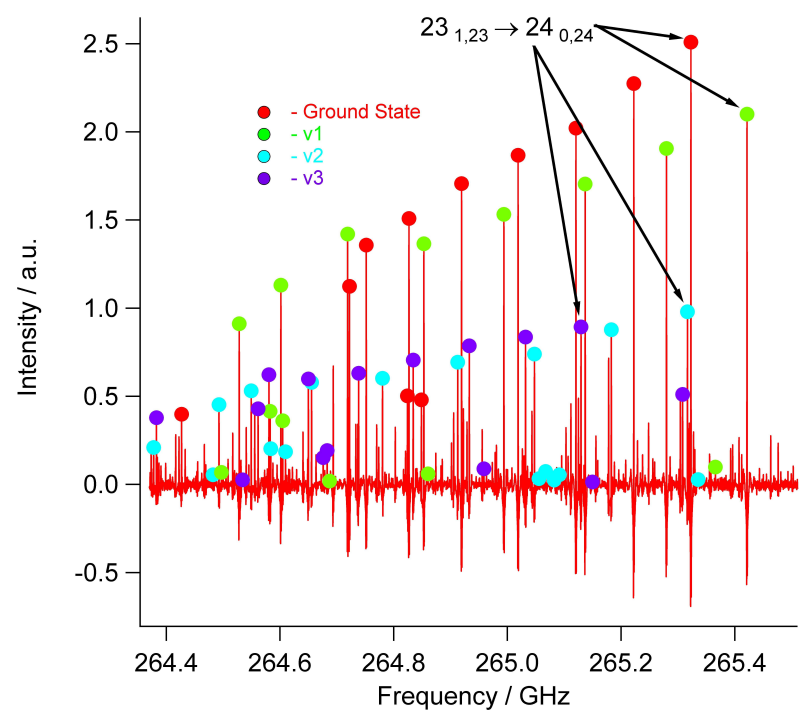

Fig. 1. Representative laboratory spectrum of urea used for spectral assignment of the four strongest vibrational states. Red, green, cyan, and purple markers represent the assigned transitions of ground, first, second, and third excited states, respectively. Quantum numbers for one transition with matching rotational quantum numbers across all four states are marked with arrows. These types of transitions were used for the calculation of the vibrational energies through spectral intensity ratios.

with rotational quantum numbers matching across vibrational states were identified at frequencies ranging from 207 to $500 \mathrm{GHz}$. Their quantum numbers $J$ and $K_{\mathrm{a}}$ range from 9 to 66 and from 0 to 31, respectively. Figure 1 shows a representative spectrum of urea with markers representing our spectroscopic assignments of the ground state and the three strongest vibrational states. The power-normalized spectra allowed us to use relative spectral intensities to calculate relative vibrational energies of the three excited vibrational states that we assigned. The relative energy of the first excited state was determined to be at $49 \pm 16 \mathrm{~cm}^{-1}$. The nature of the three excited vibrational states is not entirely clear, but quantum-chemical calculations by Inostroza \& Senent (2012) suggest that these may be the three lowest modes associated with the two large-amplitude $\mathrm{NH}_{2}$ wagging modes.

A detailed account of the analysis of this data set will be reported elsewhere (Medvedev et al., in prep.). In short, all fits were performed with the SPFIT /SPCAT package (Pickett 1991) using Watson's A-reduced asymmetric top Hamiltonian in the oblate top representation III $^{1}$ (Watson 1977). For the ground and first excited vibrational states, isolated single state fits were sufficient. The second and third vibrational states were found to be mutually perturbed and required a Coriolis-type coupled fit. Each of the three fits reproduced measured frequencies to a standard deviation of less than $50 \mathrm{kHz}$, and allowed us to predict the rest frequencies with a precision of better than $10 \mathrm{kHz}$. Both ground and first excited states were fitted with 12 effective Hamiltonian parameters which included all quartic and four sextic parameters $\left(H_{J}, H_{J K}, H_{K J}\right.$, and $\left.H_{K}\right)$.

\section{Astronomical results}

\subsection{Continuum emission}

Figure 2 shows two maps of continuum emission obtained with the ReMoCA survey at $99.2 \mathrm{GHz}$. The top and bottom maps 


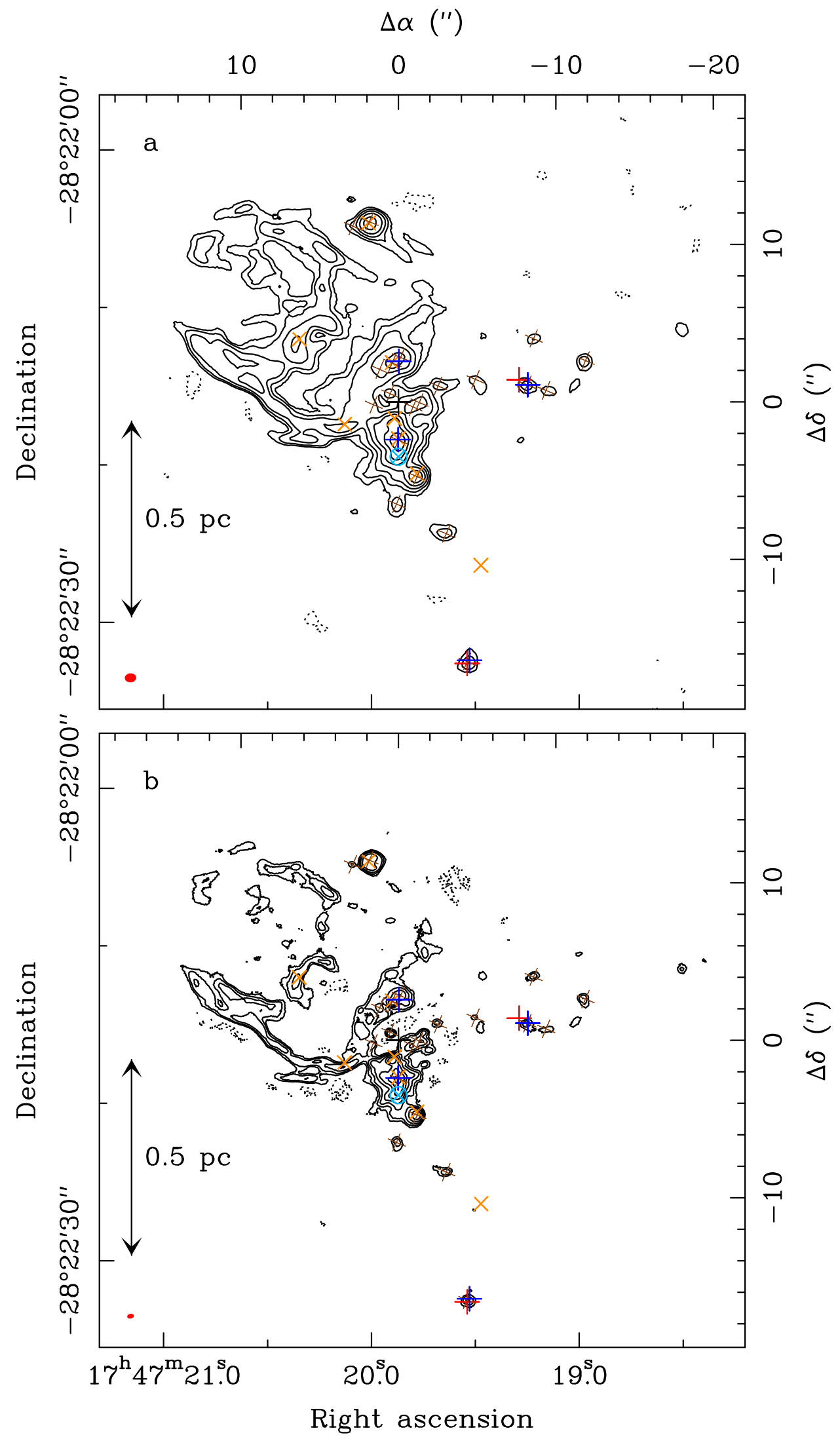

Fig. 2. Continuum map at $99.2 \mathrm{GHz}$ extracted from setups $\mathrm{S} 1(a)$ and $\mathrm{S} 5(b)$, respectively. The contours start at $4 \sigma(a)$ and $5 \sigma(b)$ with $\sigma$ the rms noise level equal to 0.95 and $0.42 \mathrm{mJy} \mathrm{beam}^{-1}$, respectively, and then increase by a factor of two. Negative contours are shown as dotted lines. In each panel, the red ellipse represents the beam (HPBW). The black cross marks the phase center, the dark blue crosses the hot cores N1-N5 reported by Bonfand et al. (2017), the light blue cross enclosed in a circle the position N1S, the brown crosses the 1 mm continuum sources detected by Sánchez-Monge et al. (2017) with ALMA, the red crosses Class II methanol masers (Caswell 1996), and the orange crosses the H II regions reported by Gaume et al. (1995). The names of some of these sources are indicated in Fig. 3. The maps are not corrected for primary-beam attenuation. 

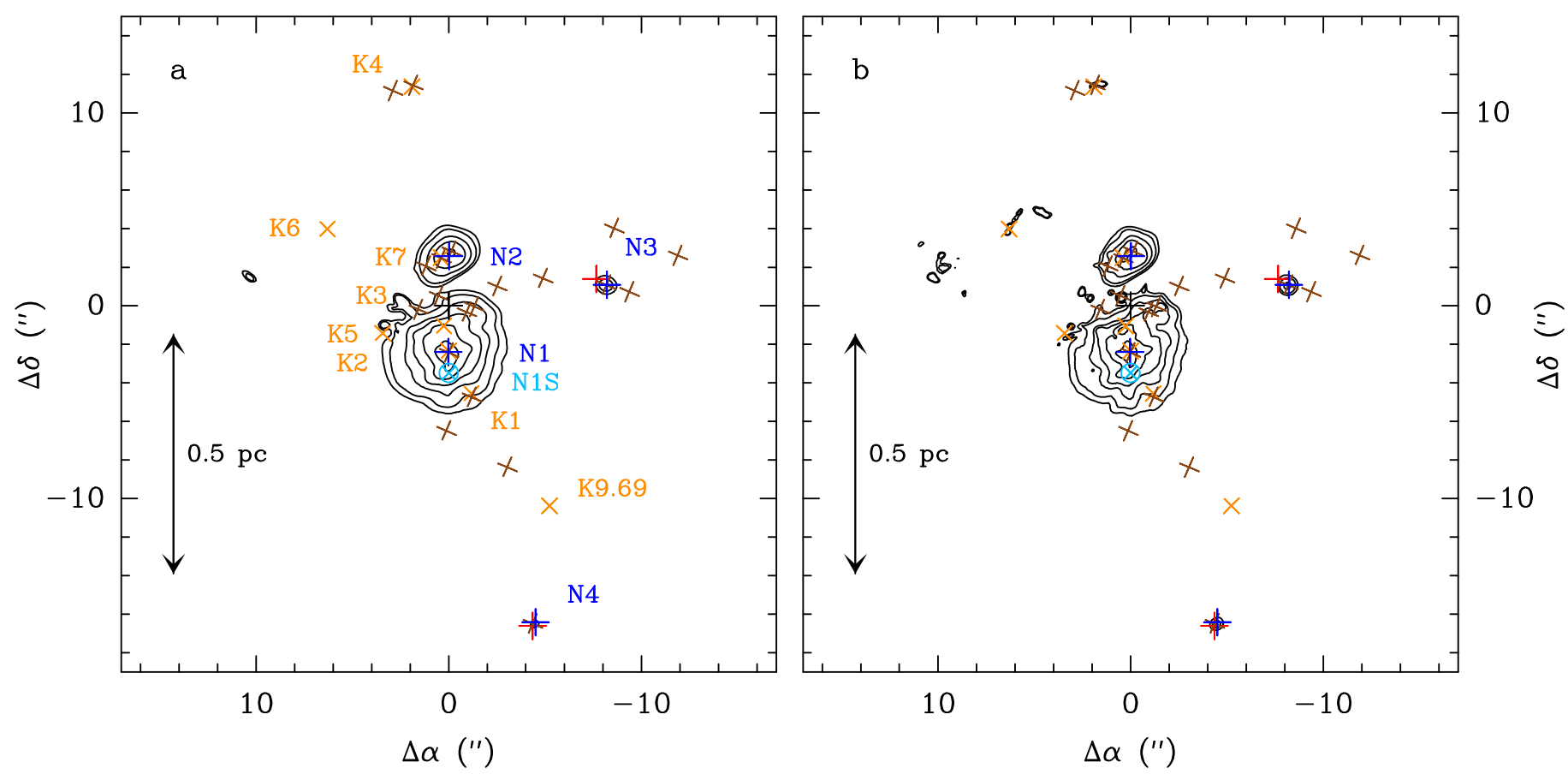

Fig. 3. Contour maps of the number of channels with continuum-subtracted flux density above the $10 \sigma$ level. (a) Only setups S1, S2, and S3, which have beam sizes on the order of $0.6^{\prime \prime}-0.7^{\prime \prime}$, were taken into account. The contours are 1000, 2000, 5000, 10000 counts, and then increase by steps of 10000 counts. (b) Only setups S4 and S5, which have beam sizes of approximately $0.4^{\prime \prime}-0.5^{\prime \prime}$, were taken into account. The contours are 700 , $1400,3500,7000$ counts, and then increase by steps of 7000 counts. The symbols in both panels are the same as in Fig. 2. The lowest contours in some parts of the maps, for instance toward $\mathrm{K} 4$ or K6, are affected by inaccuracies in the baseline subtraction and may not trace true line emission.

are extracted from setups S1 and S5 with beam sizes of $\sim 0.64^{\prime \prime}$ and $\sim 0.36^{\prime \prime}$, respectively. Although the splitting of the line and continuum emissions is still preliminary (see Sect. 2.2), the similarities between both maps give us confidence in their reliability. Many faint sources match well the positions of continuum sources detected by Sánchez-Monge et al. (2017) with ALMA at higher frequencies $(211-275 \mathrm{GHz})$ with a similar angular resolution $\left(\sim 0.4^{\prime \prime}\right)$. The ReMoCA continuum maps are also very similar to the map shown in Fig. 3 of Ginsburg et al. (2018), obtained with ALMA at $3 \mathrm{~mm}$ with a similar angular resolution $\left(\sim 0.5^{\prime \prime}\right)$.

The northeastern part of the maps shown in Fig. 2 is dominated by free-free emission from the $\mathrm{H}$ II regions $\mathrm{K} 4, \mathrm{~K} 5$, and K6, well detected at $22 \mathrm{GHz}$ with the Very Large Array (see Fig. 6 of Gaume et al. 1995) but not in the $1.3 \mathrm{~mm}$ maps of Sánchez-Monge et al. (2017) in which dust emission dominates. The extended emission detected with ReMoCA around the hot core Sgr B2(N1) does not have any counterpart in the VLA map, suggesting that it is dominated by dust emission as seen in the $1.3 \mathrm{~mm}$ maps. Compact emission is clearly detected in the ReMoCA maps toward the UCH II region K1 while it is barely seen at $1.3 \mathrm{~mm}$, suggesting that it is dominated by free-free emission in the ReMoCA maps. Free-free emission emitted by the $\mathrm{UCH}$ II regions $\mathrm{K} 2$ (coincident with $\mathrm{N} 1$ ) and $\mathrm{K} 3$ certainly also contributes to the continuum emission detected with ReMoCA.

\subsection{Map of spectral line density}

Figure 3 shows maps of channel counts, that is the number of channels with continuum-subtracted flux density above a threshold of $10 \sigma$ (for a similar map based on the EMoCA survey, see Bonfand et al. 2017). More sensitive maps with a lower threshold will be computed in the future when the splitting of the line and continuum emissions will have been finalized. Channels in the frequency ranges covered several times were counted only once. Because of the different angular resolutions of the five spectral setups, we computed two maps: the first one (Fig. 3a) using setups S1, S2, and S3 only, the second one (Fig. 3b) using setups S4 and S5 only. Several positions are affected by inaccuracies in the baseline subtraction, which in some cases leads to an overestimation of the number of channels with true line emission. This is in particular the case around the UCH II regions K4 and K6. Despite this issue, we recover the peaks detected by Bonfand et al. (2017) toward N3 and N4 that led to the discovery of these hot cores. There may be other new, compact sources in the ReMoCA channel count maps, but a closer inspection of the line-continuum splitting will be required before we can draw any firm conclusions about these faint structures.

\subsection{Position selection}

At the high angular resolution achieved with ReMoCA, the spectrum toward the continuum peak position of Sgr B2(N1) is severely affected by the optical depth of the continuum emission that masks most of the line emission. The situation is similar to that encountered for the protostellar binary IRAS 16293-2422 in the PILS survey (Jørgensen et al. 2016). As in the case of IRAS 16293-2422, we decided to analyze a position slightly offset from the dust continuum peak. We selected the position at $(\alpha, \delta)_{\mathrm{J} 2000}=\left(17^{\mathrm{h}} 47^{\mathrm{m}} 19^{\mathrm{s}} .870,-28^{\circ} 22^{\prime} 19^{\prime} .48\right)$, about $1^{\prime \prime}$ to the south of Sgr B2(N1). We call this position Sgr B2(N1S). It is shown as a light blue cross enclosed in a circle in Figs. 2 and 3. This position is sufficiently close to $\mathrm{N} 1$ to allow us to derive the chemical composition of the hot core, but still far enough to suffer less from the opacity of the dust emission. In our search for a suitable position around N1, we paid attention to the widths of the detected spectral lines. We selected N1S because of its narrow linewidths, on the order of $5 \mathrm{~km} \mathrm{~s}^{-1}$, which is as narrow as 
the width of Sgr B2(N2)'s spectral lines in the EMoCA survey. This position does not show prominent linewings as was the case for Sgr B2(N1) at poorer angular resolution in the EMoCA survey. The narrow linewidths and the absence of strong linewing emission reduce the level of spectral confusion. The ReMoCA survey thus allows us to investigate the molecular composition of N1, the main hot core of Sgr B2(N), in greater detail than was possible before with the EMoCA survey.

We followed the same method as Belloche et al. (2016) to model the spectrum of N1S. Details about the modeling can be found there. In short, we use the Weeds software (Maret et al. 2011) to produce synthetic spectra of complex organic molecules under the LTE assumption, which is well justified given the high densities that characterize Sgr B2(N)'s hot cores (Bonfand et al. 2017). We started from the list of molecules identified toward $\mathrm{N} 2$ with the EMoCA survey to build a full model of the COM emission detected with ReMoCA toward N1S. We assumed a source size of $2^{\prime \prime}$ for all species, which is more extended than the beam. While this full model is still preliminary, it fits the observed spectrum well enough to allow for a search for new molecules.

\subsection{Detection of urea}

We used the spectroscopic predictions described in Sect. 3 to search for $\mathrm{NH}_{2} \mathrm{C}(\mathrm{O}) \mathrm{NH}_{2}$ toward Sgr B2(N1S). The spectroscopic predictions were formatted in the same way as in the Cologne database for molecular spectroscopy (CDMS, Endres et al. 2016), using the same partition function values as version 1 of the CDMS entry 60517 of urea. The spectra of the transitions covered by the ReMoCA survey that are expected to be detectable are shown in Fig. A.1 for the vibrational ground state and in Fig. A.2 for the first vibrationally excited state. We assumed a temperature of $160 \mathrm{~K}$ to compute a synthetic spectrum for $\mathrm{NH}_{2} \mathrm{C}(\mathrm{O}) \mathrm{NH}_{2}$. The synthetic spectrum matches the ReMoCA spectrum relatively well, as evaluated by visual inspection, but the temperature is not well constrained, as shown below with the population diagram analysis. Many transitions of urea are blended with emission lines from other species and cannot be unambiguously identified, but nine transitions, marked with green stars in Figs. A.1 and A.2, suffer from less contamination and can be considered as clearly detected. These detected transitions are presented in a concise way in Fig. 4 and their spectroscopic parameters are listed in Table 3 . The parameters used to fit the urea spectrum are reported in Table 4.

Two transitions from within the first vibrationally excited state are in marginal agreement with the observed spectrum when their synthetic spectrum is added to the contribution of all other identified molecules. The first slight discrepancy occurs at $89.881 \mathrm{GHz}$ where the full model overestimates the detected signal by $\sim 5 \sigma$ (see Fig. A.2). Given that the full model also overestimates the two lines (of other species) around $\sim 89.87 \mathrm{GHz}$, the reason for this discrepancy is most likely an overestimation of the baseline level. The second discrepancy occurs at $\sim 102.645$ $102.650 \mathrm{GHz}$, with the full model that includes contributions from all identified molecules overestimating the detected signal by $\sim 15 \sigma$ (see blue spectrum in Fig. A.2). The urea line is blended with transitions of several species, in particular a transition of $\mathrm{C}_{2} \mathrm{H}_{5} \mathrm{CN}$ in its vibrationally excited state $v_{20}=1$ that has a large frequency uncertainty of $1.1 \mathrm{MHz}$. We suspect that the frequency of this transition is incorrect. When we remove it from the full model, the remaining discrepancy at the frequency of the urea line is reduced to $\sim 10 \sigma$. Given that the model also overestimates the observed line at $\sim 102.641 \mathrm{GHz}$ (which is a blend of $\mathrm{c}-\mathrm{C}_{2} \mathrm{H}_{4} \mathrm{O}$

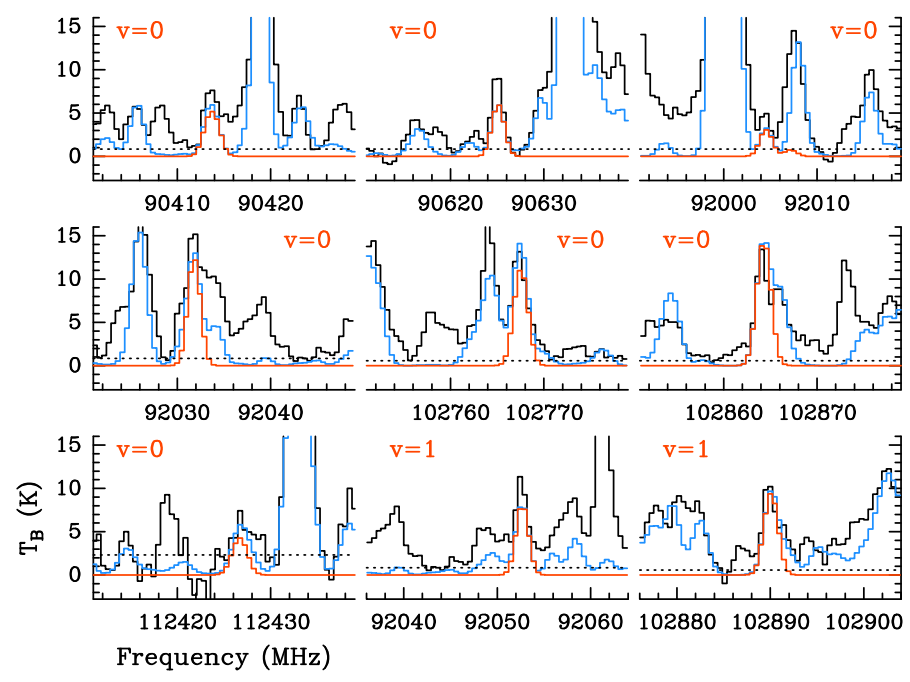

Fig. 4. Transitions of $\mathrm{NH}_{2} \mathrm{C}(\mathrm{O}) \mathrm{NH}_{2}$ in its vibrational ground state or first vibrationally excited state detected in the ReMoCA survey toward Sgr B2(N1S). The best-fit LTE synthetic spectrum of $\mathrm{NH}_{2} \mathrm{C}(\mathrm{O}) \mathrm{NH}_{2}$ is displayed in red and overlaid on the observed spectrum of Sgr B2(N1S) shown in black. The blue synthetic spectrum contains the contributions of all molecules identified in our survey so far, including $\mathrm{NH}_{2} \mathrm{C}(\mathrm{O}) \mathrm{NH}_{2}$. The dotted line indicates the $3 \sigma$ noise level.

and $n-\mathrm{C}_{3} \mathrm{H}_{7} \mathrm{CN}$, both unambiguously detected) by $\sim 11 \sigma$, hinting maybe at a slight overestimation of the baseline level, this second discrepancy is likely not to be significant. All in all, these two slight discrepancies do not invalidate our identification of urea.

In addition to the vibrational ground state and first vibrationally excited state, we also looked for transitions from within the second and third vibrationally excited states reported in Sect. 3. Unfortunately, they are either predicted to be below the $3 \sigma$ detection limit or blended with stronger lines of other species, which prevents their identification.

We also built a population diagram following the same method as Belloche et al. (2016) and using all nine detected transitions, as well as a few additional ones for which we have partly identified the contaminating species (Fig. B.1). The formal fit to this population diagram, after correction for the line optical depth and the contamination by the other identified species (Fig. B.1b), yields a rotational temperature of $350 \pm 190 \mathrm{~K}$ (see Table 5). On top of the high uncertainty, we think that this is not a reliable number. The first reason is that the population diagram method originally described by Goldsmith \& Langer (1999) requires the background continuum temperature to be negligible compared to the excitation temperature of the transitions. This is no longer the case at the high angular resolution achieved by the ReMoCA survey. An obvious sign that this assumption is not valid is the distribution of the synthetic (red) datapoints in Fig. B.1b. After correction for the line optical depth, they should fall onto a straight line, modulo a small dispersion due to the finite ranges used to integrate the line intensities, but this is not the case: the shift by a factor of 1.5 between the synthetic datapoints in the range $80-110 \mathrm{~K}$ results from these datapoints coming from setups with different angular resolutions, hence different continuum background levels. This dependence on the background level affects the observed datapoints as well and cannot be accounted for by a simple linear fit to the population diagram.

The second reason why the fitted rotational temperature is not reliable is that some transitions suffer from contamination 
Table 3. Spectroscopic parameters of urea transitions detected toward Sgr B2(N1S).

\begin{tabular}{|c|c|c|c|c|c|c|c|c|c|c|c|c|c|}
\hline \multirow{3}{*}{$\begin{array}{c}\text { Frequency } \\
(\mathrm{MHz})\end{array}$} & \multirow{3}{*}{$\begin{array}{l}\Delta f^{(a)} \\
(\mathrm{kHz})\end{array}$} & \multirow{3}{*}{$\begin{array}{c}A_{\mathrm{ul}}{ }^{(b)} \\
\left(10^{-5} \mathrm{~s}^{-1}\right)\end{array}$} & \multirow{3}{*}{$\begin{array}{r}E_{\mathrm{u}}{ }^{(c)} \\
(\mathrm{K})\end{array}$} & \multirow{3}{*}{$g_{\mathrm{u}}^{(d)}$} & \multicolumn{9}{|c|}{ Quantum numbers } \\
\hline & & & & & \multicolumn{4}{|c|}{ Upper level } & \multirow{2}{*}{$\begin{array}{l}- \\
-\end{array}$} & \multicolumn{4}{|c|}{ Lower level } \\
\hline & & & & & $J_{\mathrm{u}}$ & $K_{\mathrm{a}, \mathrm{u}}$ & $K_{\mathrm{c}, \mathrm{u}}$ & $v_{\mathrm{u}}^{(e)}$ & & $J_{1}$ & $K_{\mathrm{a}, 1}$ & $K_{\mathrm{c}, 1}$ & $v_{1}{ }^{(e)}$ \\
\hline 90413.2891 & 5.7 & 1.62 & 107.6 & 31 & 15 & 7 & 8 & 0 & - & 15 & 6 & 9 & 0 \\
\hline 90414.1278 & 5.7 & 1.62 & 107.6 & 31 & 15 & 8 & 8 & 0 & - & 15 & 7 & 9 & 0 \\
\hline 90624.9667 & 5.8 & 1.52 & 92.1 & 29 & 14 & 6 & 8 & 0 & - & 14 & 5 & 9 & 0 \\
\hline 90625.2268 & 5.8 & 1.52 & 92.1 & 29 & 14 & 7 & 8 & 0 & - & 14 & 6 & 9 & 0 \\
\hline 92004.5312 & 4.6 & 2.31 & 17.6 & 13 & 6 & 3 & 4 & 0 & - & 5 & 2 & 3 & 0 \\
\hline 92031.8092 & 6.8 & 3.79 & 20.8 & 17 & 8 & 0 & 8 & 0 & - & 7 & 1 & 7 & 0 \\
\hline 92031.8092 & 6.8 & 3.79 & 20.8 & 17 & 8 & 1 & 8 & 0 & - & 7 & 0 & 7 & 0 \\
\hline 102767.5263 & 6.4 & 4.55 & 24.6 & 17 & 8 & 1 & 7 & 0 & - & 7 & 2 & 6 & 0 \\
\hline 102767.5465 & 6.4 & 4.55 & 24.6 & 17 & 8 & 2 & 7 & 0 & - & 7 & 1 & 6 & 0 \\
\hline 102864.3049 & 7.4 & 5.37 & 25.7 & 19 & 9 & 0 & 9 & 0 & - & 8 & 1 & 8 & 0 \\
\hline 102864.3049 & 7.4 & 5.37 & 25.7 & 19 & 9 & 1 & 9 & 0 & - & 8 & 0 & 8 & 0 \\
\hline 112426.6894 & 7.0 & 2.07 & 82.9 & 29 & 14 & 4 & 10 & 0 & - & 14 & 3 & 11 & 0 \\
\hline 112426.6895 & 7.0 & 2.07 & 82.9 & 29 & 14 & 5 & 10 & 0 & - & 14 & 4 & 11 & 0 \\
\hline 92052.6888 & 7.2 & 3.79 & 92.7 & 17 & 8 & 0 & 8 & 1 & - & 7 & 1 & 7 & $\overline{1}$ \\
\hline 92052.6889 & 7.2 & 3.79 & 92.7 & 17 & 8 & 1 & 8 & 1 & - & 7 & 0 & 7 & 1 \\
\hline 102890.0663 & 7.9 & 5.38 & 97.7 & 19 & 9 & 0 & 9 & 1 & - & 8 & 1 & 8 & 1 \\
\hline 102890.0663 & 7.9 & 5.38 & 97.7 & 19 & 9 & 1 & 9 & 1 & - & 8 & 0 & 8 & 1 \\
\hline
\end{tabular}

Notes. ${ }^{(a)}$ Frequency uncertainty. ${ }^{(b)}$ Einstein coefficient for spontaneous emission. ${ }^{(c)}$ Upper-level energy. ${ }^{(d)}$ Upper-level degeneracy. ${ }^{(e)} v=0$ corresponds to the vibrational ground state and $v=1$ to the first vibrationally excited state.

Table 4. Parameters of our best-fit LTE model of urea, N-methylformamide, and other related molecules toward Sgr B2(N1S).

\begin{tabular}{|c|c|c|c|c|c|c|c|c|c|}
\hline Molecule & Status $^{(a)}$ & $N_{\text {det }}^{(b)}$ & $\begin{array}{c}\operatorname{Size}^{(c)} \\
\left({ }^{\prime \prime}\right)\end{array}$ & $\begin{array}{l}T_{\mathrm{rot}}(d) \\
(\mathrm{K})\end{array}$ & $\begin{array}{c}N^{(e)} \\
\left(\mathrm{cm}^{-2}\right)\end{array}$ & $F_{\text {vib }}{ }^{(f)}$ & $\begin{array}{c}\Delta V^{(g)} \\
\left(\mathrm{km} \mathrm{s}^{-1}\right)\end{array}$ & $\begin{array}{c}V_{\mathrm{off}}{ }^{(h)} \\
\left(\mathrm{km} \mathrm{s}^{-1}\right)\end{array}$ & $\frac{N_{\text {ref }}}{N}(i)$ \\
\hline $\mathrm{NH}_{2} \mathrm{C}(\mathrm{O}) \mathrm{NH}_{2}, v=0^{\star}$ & $\mathrm{d}$ & 7 & 2.0 & 160 & $2.7(16)$ & 1.86 & 5.0 & 0.0 & 1 \\
\hline$v=1$ & d & 2 & 2.0 & 160 & $2.7(16)$ & 1.86 & 5.0 & 0.0 & 1 \\
\hline $\mathrm{CH}_{3} \mathrm{NHCHO}, v=0^{\star}$ & $\mathrm{d}$ & 38 & 2.0 & 160 & $2.6(17)$ & 1.19 & 4.5 & 0.5 & 1 \\
\hline$v_{\mathrm{t}}=1$ & d & 15 & 2.0 & 160 & $2.6(17)$ & 1.19 & 4.5 & 0.5 & 1 \\
\hline$v_{\mathrm{t}}=2$ & d & 7 & 2.0 & 160 & $2.6(17)$ & 1.19 & 4.5 & 0.5 & 1 \\
\hline $\mathrm{CH}_{3} \mathrm{NCO}, v=0^{\star}$ & $\mathrm{d}$ & 49 & 2.0 & 200 & $2.5(17)$ & 1.00 & 5.0 & 0.0 & 1 \\
\hline$v_{\mathrm{b}}=1$ & $\mathrm{~d}$ & 2 & 2.0 & 200 & $2.5(17)$ & 1.00 & 5.0 & 0.0 & 1 \\
\hline $\mathrm{NH}_{2} \mathrm{CHO}, v=0^{\star}$ & $\mathrm{d}$ & 20 & 2.0 & 160 & $2.9(18)$ & 1.09 & 6.0 & 0.0 & 1 \\
\hline$v_{12}=1$ & d & 14 & 2.0 & 160 & $2.9(18)$ & 1.09 & 6.0 & 0.0 & 1 \\
\hline $\mathrm{NH}_{2}{ }^{13} \mathrm{CHO}, v=0$ & d & 11 & 2.0 & 200 & $1.1(17)$ & 1.17 & 5.5 & 0.0 & 28 \\
\hline$v_{12}=1$ & d & 1 & 2.0 & 200 & $1.1(17)$ & 1.17 & 5.5 & 0.0 & 28 \\
\hline $\mathrm{NH}_{2} \mathrm{CH}^{18} \mathrm{O}, v=0$ & $d$ & 2 & 2.0 & 200 & $2.1(16)$ & 1.17 & 5.5 & 0.0 & 140 \\
\hline${ }^{15} \mathrm{NH}_{2} \mathrm{CHO}, v=0$ & d & 2 & 2.0 & 200 & $9.4(15)$ & 1.17 & 5.5 & 0.0 & 314 \\
\hline $\mathrm{CH}_{3} \mathrm{C}(\mathrm{O}) \mathrm{NH}_{2}, v=0^{\star}$ & $\mathrm{d}$ & 80 & 2.0 & 160 & $4.1(17)$ & 1.16 & 5.0 & 0.0 & 1 \\
\hline$v_{\mathrm{t}}=1$ & d & 53 & 2.0 & 160 & $4.1(17)$ & 1.16 & 5.0 & 0.0 & 1 \\
\hline$v_{\mathrm{t}}=2$ & d & 18 & 2.0 & 160 & $4.1(17)$ & 1.16 & 5.0 & 0.0 & 1 \\
\hline$\Delta v_{\mathrm{t}} \neq 0$ & d & 2 & 2.0 & 160 & 4.1 (17) & 1.16 & 5.0 & 0.0 & 1 \\
\hline
\end{tabular}

Notes. ${ }^{(a)}$ d: detection. ${ }^{(b)}$ Number of detected lines (conservative estimate, see Sect. 3 of Belloche et al. 2016). One line of a given species may mean a group of transitions of that species that are blended together. ${ }^{(c)}$ Source diameter (FWHM). ${ }^{(d)}$ Rotational temperature. ${ }^{\left({ }^{(e)}\right.}$ Total column density of the molecule. $x(y)$ means $x \times 10^{y}$. An identical value for all listed vibrational/torsional states of a molecule means that LTE is an adequate description of the vibrational/torsional excitation. ${ }^{(f)}$ Correction factor that was applied to the column density to account for the contribution of vibrationally excited states, in the cases where this contribution was not included in the partition function of the spectroscopic predictions. ${ }^{(g)}$ Linewidth (FWHM). ${ }^{(h)}$ Velocity offset with respect to the assumed systemic velocity of Sgr B2(N1S), $V_{\text {sys }}=62 \mathrm{~km} \mathrm{~s}^{-1}$. ${ }^{(i)}$ Column density ratio, with $N_{\text {ref }}$ the column density of the previous reference species marked with a $\star$. 
Table 5. Rotational temperatures derived from population diagrams toward Sgr B2(N1S).

\begin{tabular}{lll}
\hline \hline \multicolumn{1}{c}{ Molecule } & \multicolumn{1}{c}{ States $^{(a)}$} & \multicolumn{1}{c}{$\begin{array}{c}T_{\mathrm{fit}}(b) \\
(\mathrm{K})\end{array}$} \\
\hline $\mathrm{NH}_{2} \mathrm{C}(\mathrm{O}) \mathrm{NH}_{2}$ & $v=0, v=1$ & $346(187)$ \\
\hline $\mathrm{CH}_{3} \mathrm{NHCHO}$ & $v=0, v_{\mathrm{t}}=1, v_{\mathrm{t}}=2$ & $187(17)$ \\
\hline $\mathrm{CH}_{3} \mathrm{NCO}$ & $v=0, v_{\mathrm{b}}=1$ & $197(24)$ \\
\hline $\mathrm{NH}_{2} \mathrm{CHO}$ & $v=0, v_{12}=1$ & $159.3(5.0)$ \\
$\mathrm{NH}_{2}{ }^{13} \mathrm{CHO}$ & $v=0, v_{12}=1$ & $284(91)$ \\
\hline $\mathrm{CH}_{3} \mathrm{CONH}$ & $v_{\mathrm{t}}=0, v_{\mathrm{t}}=1, v_{\mathrm{t}}=2, \Delta v_{\mathrm{t}} \neq 0$ & $226(13)$ \\
\hline
\end{tabular}

Notes. ${ }^{(a)}$ Vibrational states that were taken into account to fit the population diagram. ${ }^{(b)}$ The standard deviation of the fit is given in parentheses. As explained in Sect. 3 of Belloche et al. (2016) and in Sect. 4.4 of this work, this uncertainty is purely statistical and should be viewed with caution. It may be underestimated.

from still unidentified species, especially in their linewings, which leads to an overestimation of their integrated intensities. This additional uncertainty is not included in the error bars plotted in the population diagram, which are purely statistical. Finally, there may be some systematic uncertainties related to the still preliminary line-continuum splitting. Given these three reasons, the first one being the most severe, this population diagram should be viewed with caution. We rather consider it as a convenient tool to visualize in a concise way the match between the synthetic and observed spectra. We stress that the quality of this match is carefully evaluated by visual inspection of the spectra themselves which we use to optimize the model parameters.

The model that contains contributions from all the species that we identified toward Sgr B2(N2) by analyzing the EMoCA survey over the past five years fits well the ReMoCA spectrum of this source. We used this model to search for urea toward N2. No sign of urea was found and the upper limit on its column density is reported in Table 6 , assuming the same rotational temperature $(180 \mathrm{~K})$ and source size $\left(0.9^{\prime \prime}\right)$ as derived for $\mathrm{CH}_{3} \mathrm{C}(\mathrm{O}) \mathrm{NH}_{2}$ toward N2 by Belloche et al. (2017). The upper limit lies more than one order of magnitude below the column density derived for urea toward N1S.

\subsection{Confirmation of $\mathrm{N}$-methylformamide}

The ReMoCA survey provides a clear detection of $\mathrm{N}$-methyl-formamide toward N1S. The transitions covered by the survey are shown in Figs. A.3-A.5 for $v=0, v_{\mathrm{t}}=1$, and $v_{\mathrm{t}}=2$, respectively. We modeled its emission assuming a temperature of $160 \mathrm{~K}$. Like urea, many lines of $\mathrm{CH}_{3} \mathrm{NHCHO}$ are contaminated by other species, but we count sixty clearly detected lines, marked with green stars in these figures. A fit to the population diagram of $\mathrm{CH}_{3} \mathrm{NHCHO}$ shown in Fig. B.2 yields a formal rotational temperature of $187 \pm 17 \mathrm{~K}$ (see Table 5), but this diagram suffers from the same limitations as the one for urea (see Sect. 4.4). This detection confirms the presence of $\mathrm{N}$-methylformamide in the ISM, as was initially suggested by the tentative detection reported toward Sgr B2(N2) on the basis of the EMoCA survey (Belloche et al. 2017).

\subsection{Other related molecules}

In order to compare the column density derived for $\mathrm{NH}_{2} \mathrm{C}(\mathrm{O}) \mathrm{NH}_{2}$ to other COMs, we derive the column densities of $\mathrm{CH}_{3} \mathrm{NCO}, \mathrm{NH}_{2} \mathrm{CHO}$ and its isotopologs, and $\mathrm{CH}_{3} \mathrm{C}(\mathrm{O}) \mathrm{NH}_{2}$ toward N1S using the ReMoCA survey. The parameters derived from the fits are listed in Table 4 and their spectra are shown in Figs. A.6-A.17. Their population diagrams are displayed in Figs. B.3-B.8.

We ignored the transitions of formamide with opacities higher than two to build its population diagram because they cannot be modeled properly in the framework of our simple Weeds model, as can be seen in Fig. A.8 at 84.5, 87.9, or $102.1 \mathrm{GHz}$ for instance. The fit to the resulting population diagram yields a well-constrained rotational temperature of $159 \pm 5 \mathrm{~K}$ (see Fig. B.4 and Table 5) and we adopt a temperature of $160 \mathrm{~K}$ to produce the synthetic spectrum of formamide. This also motivates our choice of the temperature assumed for the synthetic spectra of urea (Sect. 4.4) and N-methylformamide (Sect. 4.5). We note that the population diagram of the ${ }^{13} \mathrm{C}$ isotopolog, especially the high energy range covered by the $v_{12}=1$ transitions, suggests a higher temperature, and we modeled this isotopolog (and the other isotopologs of formamide) assuming a temperature of $200 \mathrm{~K}$. However, it could be that the $v_{12}=1$ transitions of the ${ }^{13} \mathrm{C}$ isotopolog are all contaminated by emission lines of other unidentified species, which would bias the fit toward higher temperatures and explain the discrepancy with respect to the main isotopolog.

The ${ }^{12} \mathrm{C} /{ }^{13} \mathrm{C}$ ratio we obtain for formamide is typical for the Galactic center region, where ratios of around 20 to 30 have been reported (e.g., Müller et al. 2008; Belloche et al. 2016; Halfen et al. 2017). The detections of $\mathrm{NH}_{2} \mathrm{CH}^{18} \mathrm{O}$ and ${ }^{15} \mathrm{NH}_{2} \mathrm{CHO}$ toward Sgr B2(N1S) are to our knowledge the first interstellar detections of these isotopologs. The ${ }^{16} \mathrm{O} /{ }^{18} \mathrm{O}$ ratio of 140 is close to the value of 182 reported by Müller et al. (2016) for methanol in Sgr B2(N2). The ${ }^{14} \mathrm{~N} /{ }^{15} \mathrm{~N}$ ratio of 314 is consistent with the value of $350 \pm 50$ derived by Thiel (2019) for $\mathrm{CN}$ in the envelope of Sgr B2, but it is somewhat lower than the value of 500 derived by Belloche et al. (2016) for ethyl cyanide in Sgr B2(N2).

\subsection{Comparison of Sgr B2(N1S) and Sgr B2(N2)}

Table 4 gives the column densities obtained for Sgr B2(N1S) on the basis of the ReMoCA survey. For comparison, we report in Table 6, in addition to the ReMoCA upper limit to the column density of urea, the column densities of the other species derived for Sgr B2(N2) by Belloche et al. (2017) on the basis of the EMoCA survey. These tables yield the following abundances relative to formamide: $\mathrm{NH}_{2} \mathrm{CHO} / \mathrm{CH}_{3} \mathrm{NCO} / \mathrm{CH}_{3} \mathrm{NHCHO} /$ $\mathrm{CH}_{3} \mathrm{C}(\mathrm{O}) \mathrm{NH}_{2} / \mathrm{NH}_{2} \mathrm{C}(\mathrm{O}) \mathrm{NH}_{2}=1 / 0.086 / 0.090 / 0.14 / 0.0093$ for Sgr B2(N1S) and $1 / 0.085 / 0.038 / 0.054 /<0.00088$ for Sgr B2(N2). The two sources have exactly the same abundance of methyl isocyanate relative to formamide, while $\mathrm{N}$-methylformamide and acetamide are $\sim 2.5$ times more abundant in Sgr B2(N1S) than in Sgr B2(N2). The difference for urea is even more pronounced: urea is about one order of magnitude less abundant than $\mathrm{CH}_{3} \mathrm{NHCHO}, \mathrm{CH}_{3} \mathrm{NCO}$, and $\mathrm{CH}_{3} \mathrm{C}(\mathrm{O}) \mathrm{NH}_{2}$ in Sgr B2(N1S), while it is at least 40-100 times less abundant than these three species in Sgr B2(N2).

\section{Chemical modeling}

To simulate the chemistry of Sgr B2(N1S), we used the astrochemical kinetics code MAGICKAL (Model for Astrophysical Gas and Ice Chemical Kinetics And Layering, Garrod 2013) with a physical and chemical setup very similar to that presented by Belloche et al. (2017), for which the emphasis was the production and destruction of $\mathrm{CH}_{3} \mathrm{NCO}$ and $\mathrm{CH}_{3} \mathrm{NHCHO}$ in Sgr B2(N2). A 
Table 6. Parameters of our best-fit LTE model of selected complex organic molecules toward Sgr B2(N2).

\begin{tabular}{|c|c|c|c|c|c|c|c|c|}
\hline Molecule & $\operatorname{Status}^{(a)}$ & $N_{\text {det }}^{(b)}$ & $\begin{array}{c}\operatorname{Size}^{(c)} \\
\left({ }^{\prime \prime}\right)\end{array}$ & $\begin{array}{c}T_{\text {rot }}(d) \\
(\mathrm{K})\end{array}$ & $\begin{array}{c}N^{(e)} \\
\left(\mathrm{cm}^{-2}\right)\end{array}$ & $F_{\mathrm{vib}}(f)$ & $\begin{array}{c}\Delta V^{(g)} \\
\left(\mathrm{km} \mathrm{s}^{-1}\right)\end{array}$ & $\begin{array}{c}V_{\text {off }}{ }^{(h)} \\
\left(\mathrm{km} \mathrm{s}^{-1}\right)\end{array}$ \\
\hline $\mathrm{NH}_{2} \mathrm{C}(\mathrm{O}) \mathrm{NH}_{2}, v=0$ & $\mathrm{n}$ & 0 & 0.9 & 180 & $<2.3(15)$ & 1.95 & 5.0 & 0.5 \\
\hline $\mathrm{CH}_{3} \mathrm{NHCHO}^{(i)}$ & $\mathrm{t}$ & 5 & 0.9 & 180 & $1.0(17)$ & 1.26 & 5.0 & 0.5 \\
\hline $\mathrm{CH}_{3} \mathrm{NCO}^{(i)}$ & $\mathrm{d}$ & 64 & 1.2 & 150 & $2.2(17)$ & 1.00 & 5.0 & -0.6 \\
\hline $\mathrm{NH}_{2} \mathrm{CHO}^{(i),(j)}$ & $\mathrm{d}$ & 43 & 0.8 & 200 & $2.6(18)$ & 1.17 & 5.5 & 0.2 \\
\hline $\mathrm{CH}_{3} \mathrm{C}(\mathrm{O}) \mathrm{NH}_{2}{ }^{(i)}$ & $\mathrm{d}$ & 23 & 0.9 & 180 & $1.4(17)$ & 1.23 & 5.0 & 1.5 \\
\hline
\end{tabular}

Notes. ${ }^{(a)}$ d: detection, t: tentative detection, n: nondetection. ${ }^{(b)}$ Number of detected lines (conservative estimate, see Sect. 3 of Belloche et al. 2016). One line of a given species may mean a group of transitions of that species that are blended together. ${ }^{(c)}$ Source diameter (FWHM). ${ }^{(d)}$ Rotational temperature. ${ }^{(e)}$ Total column density of the molecule. $x(y)$ means $x \times 10^{y} \cdot{ }^{(f)}$ Correction factor that was applied to the column density to account for the contribution of vibrationally excited states, in the cases where this contribution was not included in the partition function of the spectroscopic predictions. ${ }^{(g)}$ Linewidth (FWHM). ${ }^{(h)}$ Velocity offset with respect to the assumed systemic velocity of Sgr B2(N2), $V_{\text {sys }}=74 \mathrm{~km} \mathrm{~s}^{-1} .{ }^{(i)} \mathrm{The}$ parameters were derived from the EMoCA survey by Belloche et al. (2017). ${ }^{(j)}$ For $\mathrm{NH}_{2} \mathrm{CHO}$, we report the parameters derived from the vibrationally excited state $v_{12}=1$.

Table 7. Peak simulated fractional abundances and associated temperatures.

\begin{tabular}{|c|c|c|c|c|c|c|}
\hline \multirow[t]{2}{*}{ Molecule } & \multicolumn{2}{|c|}{ Fast model } & \multicolumn{2}{|c|}{ Medium model } & \multicolumn{2}{|c|}{ Slow model } \\
\hline & $n(\mathrm{i}) / n\left(\mathrm{H}_{2}\right)$ & $\begin{array}{c}T \\
(\mathrm{~K}) \\
\end{array}$ & $n(\mathrm{i}) / n\left(\mathrm{H}_{2}\right)$ & $\begin{array}{c}T \\
(\mathrm{~K}) \\
\end{array}$ & $n(\mathrm{i}) / n\left(\mathrm{H}_{2}\right)$ & $\begin{array}{c}T \\
(\mathrm{~K}) \\
\end{array}$ \\
\hline $\mathrm{NH}_{2} \mathrm{C}(\mathrm{O}) \mathrm{NH}_{2}$ & $3.9(-8)$ & 206 & $8.3(-8)$ & 200 & $5.4(-8)$ & 191 \\
\hline $\mathrm{CH}_{3} \mathrm{NHCHO}$ & $4.4(-10)$ & 170 & $2.2(-9)$ & 165 & $1.7(-9)$ & 158 \\
\hline $\mathrm{CH}_{3} \mathrm{NCO}$ & $1.3(-8)$ & 150 & $9.4(-9)$ & 143 & $1.1(-10)$ & 131 \\
\hline $\mathrm{NH}_{2} \mathrm{CHO}$ & $1.0(-6)$ & 255 & $5.1(-7)$ & 157 & $5.9(-8)$ & 141 \\
\hline $\mathrm{CH}_{3} \mathrm{C}(\mathrm{O}) \mathrm{NH}_{2}$ & $1.7(-8)$ & 143 & $3.2(-8)$ & 138 & $2.3(-7)$ & 134 \\
\hline
\end{tabular}

Notes. $x(y)$ means $x \times 10^{y}$.

brief description of the model is given here. The reader should refer to Garrod (2013) and Belloche et al. (2014, 2017) for more detail.

\subsection{Chemical model}

The model is run in two stages: (i) a cold collapse from a gas density of $3 \times 10^{3} \mathrm{~cm}^{-3}$ to $2 \times 10^{8} \mathrm{~cm}^{-3}$, during which the gas temperature is static, while the dust temperature falls from $\sim 18$ to $8 \mathrm{~K}$; followed by (ii) a warm up from 8 to $400 \mathrm{~K}$ at fixed density, during which the gas and dust temperatures are assumed to be identical. The timescale for the initial collapse stage is approximately $10^{6} \mathrm{yr}$. For the warm-up phase, here the usual three warm-up timescales (fast, medium, and slow) are tested, corresponding to a time of $5 \times 10^{4}, 2 \times 10^{5}$, or $10^{6} \mathrm{yr}$ to reach a temperature of $200 \mathrm{~K}$. The cosmic-ray ionization rate is held at the canonical value, $\zeta=1.3 \times 10^{-17} \mathrm{~s}^{-1}$.

The chemical network, which is unchanged from that of Belloche et al. (2017), includes a full gas-phase, grain/icesurface, and ice-mantle chemistry. The choice of grainsurface activation energy barriers relating to the $\mathrm{CH}_{3} \mathrm{NCO}$ and $\mathrm{CH}_{3} \mathrm{NHCHO}$ chemistry corresponds to one of the optimal model setups presented in that publication, labeled M4B. The only change made here to that model is the adjustment of several grain-surface binding energies, most importantly, that of methyl isocyanate. In the model of Belloche et al. (2017), the desorption (binding) energy, $E_{\mathrm{des}}$, used for this molecule was $3575 \mathrm{~K}$, based on a simple additive formula using values for its constituent atoms and groups $\left(\mathrm{CH}_{3}, \mathrm{~N}, \mathrm{C}\right.$, and $\left.\mathrm{O}\right)$. That value produced a relatively low desorption temperature in the hot-core models, around $75 \mathrm{~K}$, which is substantially lower than the excitation temperature of $200 \mathrm{~K}$ derived from the observations. Recently, Bertin et al. (2017) determined desorption energies on water ice for the related molecules $\mathrm{CH}_{3} \mathrm{CN}$ and $\mathrm{CH}_{3} \mathrm{NC}$ of 6150 and $5686 \mathrm{~K}$, respectively. As it is very likely that the desorption energy of $\mathrm{CH}_{3} \mathrm{NCO}$ would be much closer to these values than to our earlier estimate, we instead use the value for methyl isocyanide $\left(\mathrm{CH}_{3} \mathrm{NC}\right)$ as a guide, to give $E_{\text {des }}\left(\mathrm{CH}_{3} \mathrm{NCO}\right)=E_{\text {des }}\left(\mathrm{CH}_{3} \mathrm{NC}\right)+E_{\text {des }}(\mathrm{O})=6486 \mathrm{~K}$. Through a similar additive method, the desorption energy for $\mathrm{N}$-methyl formamide is also raised, to a value of $7386 \mathrm{~K}$, approximately $1000 \mathrm{~K}$ higher than our previous value. As may be seen in Table 7, these values produce peak-abundance temperatures much closer to the best-fit spectral-model values adopted in the present work. The desorption energies of several related radicals, as well as those of methyl cyanide/isocyanide themselves, are adjusted similarly in this model.

\subsection{Results}

Figure 5 shows the time evolution of the simulated chemical abundances of the five observed molecules of Table 4, for each of the three warm-up scenarios (fast, medium, and slow), while Table 7 shows the peak abundances and associated model temperatures for each of the molecules and models. The peak abundances are mostly produced at the desorption temperatures of the molecules, although in the case of $\mathrm{NH}_{2} \mathrm{CHO}$, there is some gas-phase production that produces a later peak in the fast and 

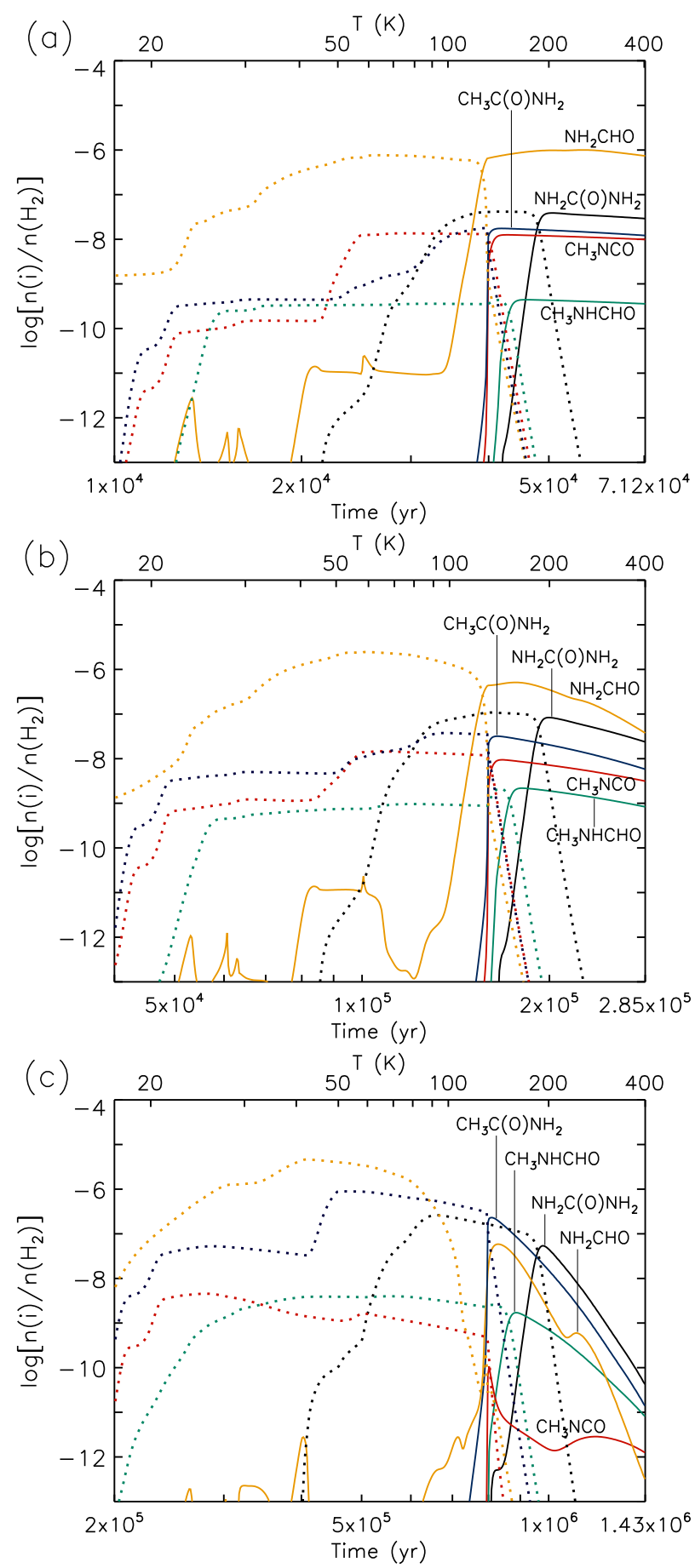

Fig. 5. Fractional abundances relative to $\mathrm{H}_{2}$ with respect to time during the warm-up stage of the astrochemical model. Solid lines indicate gas-phase abundances; dashed lines of the same color indicate the same molecules in the solid phase. Panel a: fast warm-up model; panel $b$ : medium warm-up model; panel $c$ : slow warm-up model.

medium warm-up models, through the reaction $\mathrm{NH}_{2}+\mathrm{H}_{2} \mathrm{CO} \rightarrow$ $\mathrm{NH}_{2} \mathrm{CHO}+\mathrm{H}$ proposed by Barone et al. (2015).

In the following, we compare the peak simulated abundances of the molecules listed in Table 7 with each other. The particular time (corresponding to a temperature in the frame of our chemical model) at which a molecule desorbs strongly depends on the assumption made about its desorption energy (see for instance the case of $\mathrm{CH}_{3} \mathrm{NCO}$ discussed above). Therefore, the positions of the peaks of the solid curves in Fig. 5 are uncertain. However, for most species, the peak abundance in the gas phase stays close to the abundance of the molecule in the solid phase before it desorbed, so, to zeroth order, the particular time at which it desorbs will not affect much its peak abundance in the gas phase. Given that the observed rotational temperatures of the various molecules do not differ much, we think that they in reality desorb at a similar temperature. This motivates our approach of comparing observed column density ratios to the ratios of peak abundances produced by the model in the gas phase.

In comparison to the other $\mathrm{N}$-bearing molecules listed in Table 7, the abundance of urea is higher in the model than in the observational data. However, between each of the models, its peak abundance is fairly stable, while the peak abundances of the other chemical species are more variable. In the medium model, urea is around an order of magnitude less abundant than formamide $\left(\mathrm{NH}_{2} \mathrm{CHO}\right)$, while the observations toward Sgr B2(N1S) suggest a ratio of around two orders of magnitude. The fast model better reproduces the observational ratio, while the slow model is worse. On the other hand, urea is more abundant than acetamide $\left(\mathrm{CH}_{3} \mathrm{C}(\mathrm{O}) \mathrm{NH}_{2}\right)$ in the fast and medium models, contrary to the observations, while the ratio of these two molecules in the slow model is in modest agreement with observations.

In all of the models, urea is mainly produced directly from $\mathrm{NH}_{2} \mathrm{CHO}$ in the grain-surface ices; hydrogen abstraction by radicals, including $\mathrm{NH}_{2}$ itself, produces the radical $\mathrm{NH}_{2} \mathrm{CO}$, with which $\mathrm{NH}_{2}$ reacts to form $\mathrm{NH}_{2} \mathrm{C}(\mathrm{O}) \mathrm{NH}_{2}$. Acetamide is mainly formed by a similar mechanism on the grains, ending in the addition of a methyl group. However, in the slow model, the addition of $\mathrm{NH}_{2}$ to the $\mathrm{CH}_{3} \mathrm{CO}$ radical, which is derived from acetaldehyde, gives a major boost to the abundance of acetamide on the grains at around $50 \mathrm{~K}$ and is responsible for its greater abundance in the gas phase once the solid-phase material has desorbed.

The observations suggest that methyl isocyanate and $\mathrm{N}$-methylformamide should be similar in abundance, but the models produce a range of ratios. The medium model comes closest to the observed ratio between the two, as well as to the ratios with other molecules such as formamide and acetamide. The adjustment to the binding energy of $\mathrm{CH}_{3} \mathrm{NCO}$ provides something of an improvement to the model results, allowing a somewhat higher abundance to be achieved than in the models of Belloche et al. (2017), while also providing a more plausible temperature of peak abundance in comparison to the observational excitation temperature.

\section{Discussion}

\subsection{Astronomical detection}

We presented in Sect. 4.4 an identification of urea that is based on the assignment of nine clearly detected lines (see Fig. 4). While there is no strict criterion to decide how many lines are necessary to have a robust detection of a molecule in emission in a nearly confusion-limited spectrum, we consider that our detailed modeling of the full spectrum of Sgr B2(N1S) that includes contributions from all molecules identified so far makes the detection of these nine lines, which are well reproduced by our LTE model of the urea emission, a sufficient number to secure the identification of the molecule. For comparison, we claimed only a tentative detection of $\mathrm{N}$-methylformamide toward Sgr B2(N2) in our previous work using the EMoCA survey because there were only five clearly detected lines assigned to this molecule (Belloche et al. 2017). With now sixty lines 
of N-methylformamide identified in the ReMoCA spectrum of Sgr B2(N1S), the identification of N-methylformamide in the ISM is robustly confirmed.

Xue et al. (2019) presented a quantitative method to evaluate the degree to which a line can be claimed as clearly assigned to a molecule. While we appreciate the efforts made to propose a quantitative estimator (their $\mathrm{P}$ and $\mathrm{D}$ factors), we did not attempt to apply this method here for two reasons. First, this method does not take into account the systematic uncertainties that may affect the observed spectra, such as the uncertainty related to the level of the baseline in a spectrum that is as close to the confusion limit as the spectrum we analyzed here, or the uncertainty related to the attenuation by the dust, which we account for in our radiative transfer model as well as we can. A second limitation of the method of Xue et al. (2019) is that the frequency range over which the $\mathrm{P}$ and $\mathrm{D}$ factors are computed for each line is, as stated by the authors themselves, crucial for calculating these factors. There is some degree of arbitrariness in the selection of these frequency ranges, especially in the case of confusionlimited spectra where (partial) blends frequently occur. Rather than relying blindly on these quantitative estimators, we consider that the visualization of the nine lines clearly assigned to urea in Fig. 4 along with the LTE synthetic spectrum of urea and the synthetic spectrum that includes contributions from all molecules are sufficient to assess the robustness of the identification.

Remijan et al. (2014) attempted a fit to the data set of their multitelescope search for urea mentioned in Sect. 1. They obtained a column density of $\sim 8 \times 10^{14} \mathrm{~cm}^{-2}$ for urea toward Sgr B2(N1), assuming a rotational temperature of $\sim 80 \mathrm{~K}$ and a source size between $2^{\prime \prime}$ and $3.25^{\prime \prime}$ depending on the telescope. This column density is more than one order of magnitude lower than the column density we derive from the ReMoCA survey for a source size of $2^{\prime \prime}$. A fit of the ReMoCA data with a temperature of $80 \mathrm{~K}$ does not significantly change the column density that we derive for urea. An explanation for the discrepancy between Remijan et al.'s result and ours could be that the urea emission region is more compact than the size that they and we assumed. Because of the small beam of the ReMoCA survey, reducing the size of the urea emission region by a factor of two would increase its column density by $40 \%$ only, while it would imply a column density a factor of four times higher in Remijan et al.'s analysis. This would reduce the discrepancy by a factor of three, which is not sufficient to resolve it.

Another possibility is that there is an issue with the calculation of the column density reported by Remijan et al. (2014) or with the spectroscopic predictions they used. To test this hypothesis, we used the spectroscopic predictions obtained in Sect. 3 to compute a synthetic spectrum for the IRAM $30 \mathrm{~m}$ telescope as Remijan et al. (2014) did in their Table 2. With their parameters (a source size of 3.25", a linewidth of $7 \mathrm{~km} \mathrm{~s}^{-1}$, a column density of $8 \times 10^{14} \mathrm{~cm}^{-2}$, a temperature of $80 \mathrm{~K}$, and a beam size of $20.2^{\prime \prime 3}$ ), we obtain a peak brightness temperature of $69 \mathrm{mK}$ at $102.864 \mathrm{GHz}$, about $15 \%$ lower than predicted by Remijan et al. (2014) at this frequency. The origin of this difference is unclear, but it is small and does not explain the discrepancy mentioned in the previous paragraph.

A third reason is that we account for the contribution of vibrationally excited states to the partition function of urea, while Remijan et al. (2014) did not. This increases the column density that we derive for urea by a factor of 1.9 at a temperature of $160 \mathrm{~K}$ (see Table 4). Ignoring this vibrational correction would reduce

3 This angular size underestimates the actual beam size (HPBW) of the IRAM $30 \mathrm{~m}$ telescope, which is about $23.9^{\prime \prime}$ at $102.9 \mathrm{GHz}$. the discrepancy by a factor of 1.9 , which is insufficient to resolve it completely.

Finally, another reason why our model delivers a higher column density is that we take into account the strong background continuum emission in the radiative transfer equation. For instance, the continuum level toward $\mathrm{Sgr}$ B2(N1S) at $102.864 \mathrm{GHz}$ is $50 \mathrm{~K}$ in the ReMoCA survey, while the observed line has a peak of $\sim 13 \mathrm{~K}$ only. A fit of the ReMoCA line at $102.864 \mathrm{GHz}$ without accounting for the background continuum emission yields a column density a factor of 1.5 lower. Because the continuum emission is even more opaque toward the peak of Sgr B2(N1), observations targeting this peak with a larger beam such as the ones performed by Remijan et al. (2014) will collect more dust-attenuated urea emission and thereby underestimate the true strength of the urea lines even further.

Combined together, these four reasons may explain the discrepancy between the column density of urea derived from the ReMoCA survey and the column density claimed by Remijan et al. (2014) for this molecule.

A point of concern could be the potential contamination of the line we assigned to urea at $92031.8 \mathrm{MHz}$ by the $\mathrm{H} \alpha 41$ recombination line at $92034.4 \mathrm{MHz}$, as briefly mentioned by Remijan et al. (2014). This recombination line is prominent in the IRAM $30 \mathrm{~m}$ spectrum of Sgr B2(N1) at a poor angular resolution of $\sim 27^{\prime \prime}$ (see Fig. 2 of Belloche et al. 2013). Figure 6 shows the portions of the ReMoCA spectrum of Sgr B2(N1S) that cover the frequencies of all $\mathrm{H} \alpha$ recombination lines that fall in the ReMoCA band. The synthetic spectrum of $\mathrm{C}_{2} \mathrm{H}_{3} \mathrm{CN} v_{11}=1$, which dominates the emission detected around the frequency of the $\mathrm{H} \alpha 42$ recombination line, is displayed in red in Fig. 6a. Our preliminary model obviously underestimates the strength of the four $\mathrm{C}_{2} \mathrm{H}_{3} \mathrm{CN}$ lines in this portion of the ReMoCA survey. If we rescale it to fit the observed line strengths then there is no room left for $\mathrm{H} \alpha 42$ emission. Likewise, we do not detect any obvious $\mathrm{H} \alpha 40$ emission in Fig. 6c, with no signal detected above $2 \mathrm{~K}$ at the rest frequency of this transition. The four spectra of Fig. 6 are displayed with the same velocity axis. Therefore, any $\mathrm{H} \alpha$ emission present toward Sgr B2(N1S) should manifest itself with the same lineshape in each panel. No such pattern is obvious in this figure. We can thus safely conclude that at the high angular resolution of the ReMoCA survey and toward the specific position Sgr B2(N1S), the ReMoCA spectrum is not significantly contaminated by $\mathrm{H} \alpha$ emission. We conclude that the assignment of the transition at $92031.8 \mathrm{MHz}$ to urea is robust and certainly not affected by any contamination from $\mathrm{H} \alpha$ emission. The prominent $\mathrm{H} \alpha$ emission seen in the single-dish spectrum around this frequency is either filtered out by the interferometer, or arises from regions that do not enclose Sgr B2(N1S).

\subsection{Chemical modeling}

In general, the model with the medium warm-up timescale appears best to reproduce the observational relative abundances of the five molecules of interest, considering that the peak absolute abundance of urea varies little between the models. The large relative abundance of urea in all cases suggests that the production mechanism for this molecule may be too efficient on the grains. Solid-phase urea is seen to be produced most strongly after around $55 \mathrm{~K}$, which is the temperature at which production and diffusion of $\mathrm{NH}_{2}$ becomes rapid. Production of $\mathrm{NH}_{2}$ is caused by the abstraction of $\mathrm{H}$ from solid-phase ammonia by $\mathrm{OH}$ radicals (see Garrod 2013), although the activation barriers and rates for such processes are poorly constrained. However, a somewhat higher barrier to the abstraction of $\mathrm{H}$ from $\mathrm{NH}_{3}$ 


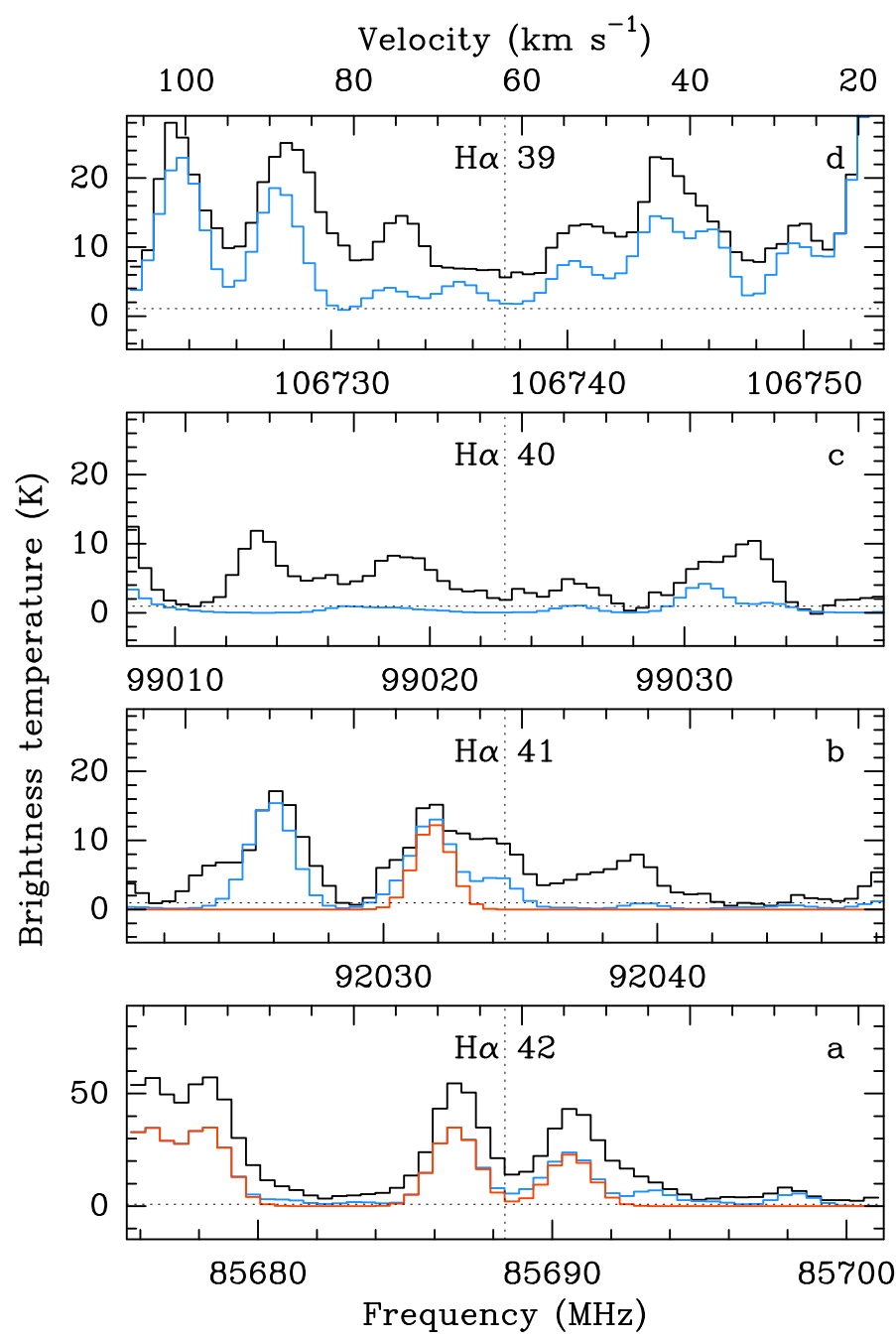

Fig. 6. Portions of the continuum-removed ReMoCA spectrum of $\mathrm{Sgr} \mathrm{B} 2(\mathrm{~N} 1 \mathrm{~S})$ that cover the frequencies of $\mathrm{H} \alpha$ recombination lines. The frequency of each $\mathrm{H} \alpha$ line is marked with a dotted line in each panel and the name of the transition is indicated. The lower and upper axes of each panel are labeled in frequency and velocity, respectively, assuming a systemic velocity of $62 \mathrm{~km} \mathrm{~s}^{-1}$. The velocity range is the same for all panels. The blue spectrum represents our current (preliminary) LTE model that includes contributions from all identified molecules. It is overlaid on the ALMA spectrum displayed in black. The red spectrum in panel $a$ is our current LTE synthetic spectrum of $\mathrm{C}_{2} \mathrm{H}_{3} \mathrm{CN}, v_{11}=1$ while the red spectrum in panel $b$ is our best-fit LTE synthetic spectrum of urea.

may be a possible solution to lower the total urea production. Although this might also reduce the production of acetamide to some degree, the latter molecule has other formation pathways, especially at lower temperatures, and thus would likely be less affected, whereas all significant urea production in the present models comes from the $\mathrm{NH}_{2}+\mathrm{NH}_{2} \mathrm{CO}$ route. The interstellar detection of urea may therefore give further constraint to the chemistry of ammonia-related species.

The production of urea through UV-induced solid-phase chemistry was recently studied as part of an investigation into the formation of peptide-like molecules on interstellar grains (Ligterink et al. 2018). These authors found that UV irradiation of $\mathrm{CH}_{4}: \mathrm{HNCO}$ ice mixtures could produce several of the species detected observationally in the present work, with the $\mathrm{NH}_{2} \mathrm{CO}$ radical being a key intermediary, in agreement with our model findings. Ligterink et al. (2018) suggested that, in their experiments, this radical should be produced by the reaction $\mathrm{NH}_{2}+$ $\mathrm{CO} \rightarrow \mathrm{NH}_{2} \mathrm{CO}$. This reaction is included in our chemical network, with an activation energy barrier arbitrarily set to $2500 \mathrm{~K}$, in line with typical values for the reaction of $\mathrm{H}$ with $\mathrm{CO}$ (Garrod et al. 2008). In the models, the reaction is found to be negligible compared with both the abstraction of hydrogen from $\mathrm{NH}_{2} \mathrm{CHO}$ and (at lower temperature) the addition of $\mathrm{H}$ to $\mathrm{HNCO}$, although the adoption of a lower barrier could change this result.

Ligterink et al. (2018) also state that the hydrogenation of $\mathrm{CH}_{3} \mathrm{NCO}$ to $\mathrm{N}$-methylformate is not consistent with their nondetection of the latter molecule (in spite of detecting the former). The models of Belloche et al. (2017) suggested that either this process or the direct addition of radicals to form $\mathrm{CH}_{3} \mathrm{NHCHO}$ are plausible to explain observations, although in the present models hydrogenation dominates. The toxicity of methyl isocyanate makes experimentation with its pure ice a challenging prospect, so unambiguous evidence of the efficacy or otherwise of this route is not yet forthcoming.

Although our models do not consider mechanisms for native gas-phase production of urea, a theoretical study by Jeanvoine \& Spezia (2019) recently considered the possible reaction between protonated hydroxylamine $\left(\mathrm{NH}_{2} \mathrm{OH}_{2}{ }^{+}\right)$and formamide $\left(\mathrm{NH}_{2} \mathrm{CHO}\right)$ to form protonated urea, which, if formed, could be converted to neutral urea through electronic recombination. Those authors found that the structure of the ionic product (with the correct mass) resulting from the suggested reaction would be unlikely ultimately to produce urea without significant structural re-arrangement; however, they suggest that the process may be more efficient if occurring in or upon an ice mantle. No astrochemical models currently exist that explicitly treat ion-molecule chemistry in the solid phase.

As mentioned in Sect. 5.2, in comparison to related chemical species, the peak abundance of urea obtained with the models presented here is somewhat higher than the observations would seem to support. However, one further destruction mechanism in the gas phase that is not currently included in the chemical network might ameliorate this disagreement. The efficiency of electronic dissociative recombination of protonated molecules in producing the neutral molecule plus a hydrogen atom is generally found only to be on the order of a few percent for the larger highly-saturated complex organic molecules for which experiments have been conducted (e.g., Geppert et al. 2006; Hamberg et al. 2010). This means that the gas-phase protonation of complex organics typically leads to the destruction of such molecules, thus limiting their lifetimes and fractional abundances. Taquet et al. (2015) suggested that protonated complex organics may instead react efficiently with neutral ammonia, passing on the proton and leaving the structure of the larger molecule intact. This mechanism is not included in the network used here. However, the thermodynamic viability of the process is dependent on the neutral complex molecule having a lower proton affinity than ammonia. The most recent determinations of this quantity for urea (207.8 $\mathrm{kcal} \mathrm{mol}^{-1}$; Zheng \& Cook 2002) and ammonia (204.0 $\mathrm{kcal} \mathrm{mol}^{-1}$; Hunter \& Lias 1998) indicate that this mechanism would have a substantial endothermicity $(\sim 1900 \mathrm{~K})$ for this pairing. Indeed, the reverse process would be energetically favorable, suggesting that neutral urea should instead be protonated by an encounter with $\mathrm{NH}_{4}{ }^{+}$, leading to an enhanced destruction rate over other complex organics. The proton affinity of acetamide (206.4 $\mathrm{kcal} \mathrm{mol}^{-1}$; Hunter \& Lias 1998) suggests that it too should behave in this way. The rates of neither the forward nor the reverse process are well studied in general, and the relatively small difference in proton affinity between urea 
and ammonia (compared with other possible pairings) means that the efficiency of proton transfer from the latter molecule to the former should perhaps be determined using more detailed methods than the crude thermodynamic argument above. However, even the simple absence of a proton-transfer mechanism between protonated urea and ammonia (due to its endothermicity), without the inclusion of the reverse mechanism, may be sufficient to decrease the relative abundance of urea found in the models versus other complex organics, if the protonated forms of those species suffer no such difficulty in reacting with ammonia. This would also make such abundances strongly dependent on the gas-phase abundance of ammonia in individual sources. A greater abundance of gas-phase ammonia (and thus of protonated ammonia) toward Sgr B2(N2), versus Sgr B2(N1S), could be one explanation for the lower abundance of urea toward N2.

Raunier et al. (2004) claimed a tentative identification of urea and formamide in interstellar ices toward the protostellar source NGC 7538 IRS9 (see Sect. 1). Unfortunately, they do not make any quantitative statement about the relative abundance of these two molecules in that source. Our best-fit chemical model for Sgr B2(N1S) (Fig. 5b) suggests that the abundance of formamide with respect to urea in the ices should be even higher than in the gas phase so, given the abundance ratio we measure in the gas phase toward Sgr B2(N1S), we expect urea to be at least two orders of magnitude less abundant than formamide in the ice mantles. It would be interesting to test whether the tentative identifications claimed by Raunier et al. (2004) in the ices are consistent with such a low abundance of urea relative to formamide.

\section{Conclusions}

The high angular resolution of the ReMoCA spectral line survey recently conducted with ALMA toward Sgr B2(N) allows us to beat the spectral confusion limit that our previous survey, EMoCA, reached toward the main hot core embedded in Sgr B2(N). One difficulty that arises, though, is that, even at $3 \mathrm{~mm}$, the dust emission starts to be optically thick on these small scales ( $\sim 4000 \mathrm{au})$, thus obscuring the molecular line emission and preventing us from deriving the molecular composition of the very inner region. However, a careful inspection of the datacube has revealed that the molecular lines detected toward the position Sgr B2(N1S), which is offset from the continuum peak by $\sim 8000$ au and therefore suffers less from the dust obscuration, have full widths at half maximum of only $\sim 5 \mathrm{~km} \mathrm{~s}^{-1}$. This is similar to the linewidths that characterize the spectrum of the secondary hot core Sgr B2(N2). In the same way as EMoCA allowed us to make significant progress in the determination of the molecular composition of Sgr B2(N2), ReMoCA now allows us to detect less abundant species toward the main hot core of Sgr B2(N). We took this opportunity to search for urea toward this source. The main results of this article are the following:

1. The rotational spectrum of urea was revisited and the ground as well as three low-lying excited vibrational states were analyzed. The results of this spectroscopic study, which will be reported separately, were instrumental for the present search for urea in space.

2. We report the identification of urea toward Sgr B2(N1S) with nine clearly detected lines in its vibrational ground state and first vibrationally excited state. This is the first secure detection of urea in the ISM.

3. We confirm the interstellar detection of N-methylformamide, which was recently tentatively identified on the basis of the EMoCA survey. Sixty lines of this molecule are clearly detected toward Sgr B2(N1S) in the ReMoCA data.

4. We report the first interstellar detections of $\mathrm{NH}_{2} \mathrm{CH}^{18} \mathrm{O}$ and ${ }^{15} \mathrm{NH}_{2} \mathrm{CHO}$.

5. We find that urea is about two orders of magnitude less abundant than formamide toward Sgr B2(N1S), and about one order of magnitude less abundant than $\mathrm{N}$-methylformamide, methyl isocyanate, and acetamide, which have similar column densities.

6. Urea is not detected toward the secondary hot core Sgr B2(N2). The nondetection implies that urea is at least one order of magnitude less abundant in Sgr B2(N2) than in Sgr B2(N1S) with respect to the other four species.

7. Our chemical modeling roughly reproduces the relative abundances of formamide, $\mathrm{N}$-methylformamide, methyl isocyanate, and acetamide measured in Sgr B2(N1S) and Sgr B2(N2). However, it overproduces urea by at least one order of magnitude. An insufficiently high barrier to hydrogen abstraction from solid-phase ammonia by $\mathrm{OH}$ radicals may be the reason for this overproduction. The likely absence of an efficient gas-phase proton-transfer mechanism from protonated urea to ammonia could also result in more rapid destruction of urea than other complex organics.

The differences in the chemical composition of Sgr B2(N1S) and Sgr B2(N2) with urea being at least one order of magnitude less abundant with respect to the other four species in the latter source is puzzling and not yet understood. A careful comparison of the entire COM composition of both sources may help us to find out the reasons for this astonishing difference.

Acknowledgements. We thank the referee, Anthony Remijan, for his critical review of our article that helped to clarify several aspects of our analysis. This paper makes use of the following ALMA data: ADS/JAO. ALMA\#2016.1.00074.S. ALMA is a partnership of ESO (representing its member states), NSF (USA), and NINS (Japan), together with NRC (Canada), NSC and ASIAA (Taiwan), and KASI (Republic of Korea), in cooperation with the Republic of Chile. The Joint ALMA Observatory is operated by ESO, AUI/NRAO, and NAOJ. The interferometric data are available in the ALMA archive at https://almascience.eso.org/aq/. Part of this work has been carried out within the Collaborative Research Centre 956, sub-project B3, funded by the Deutsche Forschungsgemeinschaft (DFG) - project ID 184018867.

\section{References}

Barone, V., Latouche, C., Skouteris, D., et al. 2015, MNRAS, 453, L31 Belloche, A., Menten, K. M., Comito, C., et al. 2008, A\&A, 482, 179 Belloche, A., Garrod, R. T., Müller, H. S. P., et al. 2009, A\&A, 499, 215 Belloche, A., Müller, H. S. P., Menten, K. M., Schilke, P., \& Comito, C. 2013, A\&A, 559, A47

Belloche, A., Garrod, R. T., Müller, H. S. P., \& Menten, K. M. 2014, Science, 345, 1584

Belloche, A., Müller, H. S. P., Garrod, R. T., \& Menten, K. M. 2016, A\&A, 587, A91

Belloche, A., Meshcheryakov, A. A., Garrod, R. T., et al. 2017, A\&A, 601, A49 Bergner, J. B., Öberg, K. I., Garrod, R. T., \& Graninger, D. M. 2017, ApJ, 841, 120

Bertin, M., Doronin, M., Fillion, J.-H., et al. 2017, A\&A, 598, A18

Bonfand, M., Belloche, A., Menten, K. M., Garrod, R. T., \& Müller, H. S. P. 2017, A\&A, 604, A60

Brown, R. D., Godfrey, P. D., \& Storey, J. 1975, J. Mol. Spectr., 58, 445

Caswell, J. L. 1996, MNRAS, 283, 606

Endres, C. P., Schlemmer, S., Schilke, P., et al. 2016, J. Mol. Spectr., 327, 95 Fosnight, A. M., Moran, B. L., \& Medvedev, I. R. 2013, Appl. Phys. Lett., 103, 133703

Fourré, I., Rosset, L., Chevreau, H., \& Ellinger, Y. 2016, A\&A, 589, A18 Garrod, R. T. 2013, ApJ, 765, 60

Garrod, R. T., Widicus Weaver, S. L., \& Herbst, E. 2008, ApJ, 682, 283 Gaume, R. A., Claussen, M. J., de Pree, C. G., et al. 1995, ApJ, 449, 663 Geppert, W. D., Hamberg, M., Thomas, R. D., et al. 2006, Faraday Discuss., 133, 177

Ginsburg, A., Bally, J., Barnes, A., et al. 2018, ApJ, 853, 171 
A. Belloche et al.: Re-exploring molecular complexity with ALMA (ReMoCA): interstellar detection of urea

Godfrey, P. D., Brown, R. D., \& Hunter, A. N. 1997, J. Mol. Spectr., 413, 405

Goldsmith, P. F., \& Langer, W. D. 1999, ApJ, 517, 209

Halfen, D. T., Woolf, N. J., \& Ziurys, L. M. 2017, ApJ, 845, 158

Hamberg, M., Zhaunerchyk, V., Vigren, E., et al. 2010, A\&A, 522, A90

Hayatsu, R., Studier, M. H., Moore, L. P., \& Anders, E. 1975, Geochim. Cosmochim. Acta, 39, 471

Herbst, E., \& van Dishoeck, E. F. 2009, ARA\&A, 47, 427

Hunter, E. P., \& Lias, S. G. 1998, J. Phys. Chem. Ref. Data, 27, 413

Inostroza, N., \& Senent, M. L. 2012, Chem. Phys. Lett., 524, 25

Jørgensen, J. K., van der Wiel, M. H. D., Coutens, A., et al. 2016, A\&A, 595, A117

Jeanvoine, Y., \& Spezia, R. 2019, Theor. Chem. Acc., 138, 1

Kassi, S., Petitprez, D., \& Wlodarczak, G. 2004, J. Mol. Spectr., 228, 293

Kasten, W., \& Dreizler, H. 1986, Z. Naturforsch. A, 41, 1173

Kisiel, Z., Thomas, J., \& Medvedev, I. 2014, 69th International Symposium on Molecular Spectroscopy, TA10

Kretschmer, U., Consalvo, D., Knaack, A., et al. 1996, Mol. Phys., 87, 1159

Ligterink, N. F. W., Terwisscha van Scheltinga, J., Taquet, V., et al. 2018, MNRAS, 480, 3628

Maret, S., Hily-Blant, P., Pety, J., Bardeau, S., \& Reynier, E. 2011, A\&A, 526, A47

McGuire, B. A. 2018, ApJS, 239, 17

Medvedev, I. R., Neese, C. F., Plummer, G. M., et al. 2010, Opt. Lett., 35, 1533
Müller, H. S. P., Belloche, A., Menten, K. M., et al. 2008, J. Mol. Spectr., 251, 319

Müller, H. S. P., Belloche, A., Xu, L.-H., et al. 2016, A\&A, 587, A92

Nuevo, M., Bredehöft, J. H., Meierhenrich, U. J., d'Hendecourt, L., \& Thiemann,

W. H.-P. 2010, Astrobiology, 10, 245

Pickett, H. M. 1991, J. Mol. Spectr., 148, 371

Qin, S.-L., Schilke, P., Rolffs, R., et al. 2011, A\&A, 530, L9

Raunier, S., Chiavassa, T., Duvernay, F., et al. 2004, A\&A, 416, 165

Reid, M. J., Menten, K. M., Brunthaler, A., et al. 2014, ApJ, 783, 130

Remijan, A. J., Snyder, L. E., McGuire, B. A., et al. 2014, ApJ, 783, 77

Remijan, A., Adams, M., \& Warmels, R. 2015, ALMA Cycle 3 Technical Handbook Version 1.0, ALMA

Sánchez-Monge, Á., Schilke, P., Schmiedeke, A., et al. 2017, A\&A, 604, A6

Taquet, V., López-Sepulcre, A., Ceccarelli, C., et al. 2015, ApJ, 804, 81

Thiel, V. 2019, PhD Thesis, University of Bonn, Bonn, Germany

Thiel, V., Belloche, A., Menten, K. M., et al. 2019, A\&A, 623, A68

Thomas, J. R., Fosnight, A. M., \& Medvedev, I. R. 2013, 68th International Symposium on Molecular Spectroscopy, WF14

Thomas, J., Medvedev, I., \& Kisiel, Z. 2014, 69th International Symposium on Molecular Spectroscopy, TA09

Watson, J. K. G. 1977, in Vibrational Spectra and Structure, ed. J. R. Durig (New York: Elsevier), 6, 1

Wöhler, F. 1828, Ann. Phys., 88, 253

Xue, C., Remijan, A. J., Burkhardt, A. M., et al. 2019, ApJ, 871, 112

Zheng, X., \& Cooks, R. G. 2002, J. Phys. Chem. A, 2002, 9939 
Appendix A: Complementary figures: spectra

Figures A.1-A.17 show the transitions of $\mathrm{NH}_{2} \mathrm{C}(\mathrm{O}) \mathrm{NH}_{2}$, $\mathrm{CH}_{3} \mathrm{NHCHO}, \mathrm{CH}_{3} \mathrm{NCO}, \mathrm{NH}_{2} \mathrm{CHO}, \mathrm{CH}_{3} \mathrm{C}(\mathrm{O}) \mathrm{NH}_{2}$, and some of their isotopologs or vibrationally excited states that are covered by the ReMoCA survey and contribute significantly to the signal detected toward Sgr B2(N1S).

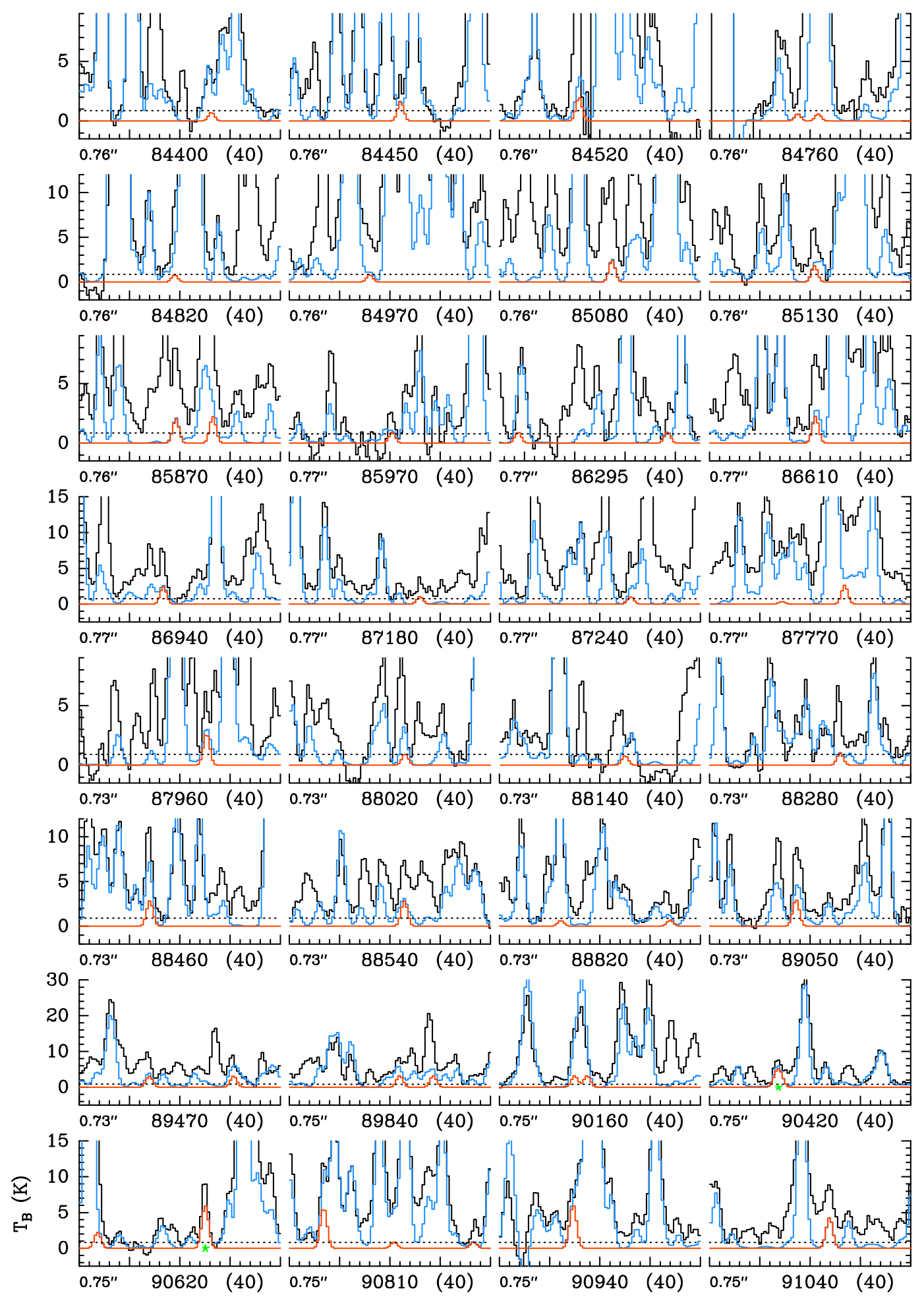

Fig. A.1. Transitions of $\mathrm{NH}_{2} \mathrm{C}(\mathrm{O}) \mathrm{NH}_{2}, v=0$ covered by our ALMA survey. The best-fit LTE synthetic spectrum of $\mathrm{NH}_{2} \mathrm{C}(\mathrm{O}) \mathrm{NH}_{2}, v=0$ is displayed in red and overlaid on the observed spectrum of Sgr B2(N1S) shown in black. The blue synthetic spectrum contains the contributions of all molecules identified in our survey so far, including the species shown in red. The central frequency and width are indicated in MHz below each panel. The angular resolution (HPBW) is also indicated. The $y$-axis is labeled in brightness temperature units (K). The dotted line indicates the $3 \sigma$ noise level. The green stars mark the transitions that we consider as clearly detected. 
A. Belloche et al.: Re-exploring molecular complexity with ALMA (ReMoCA): interstellar detection of urea
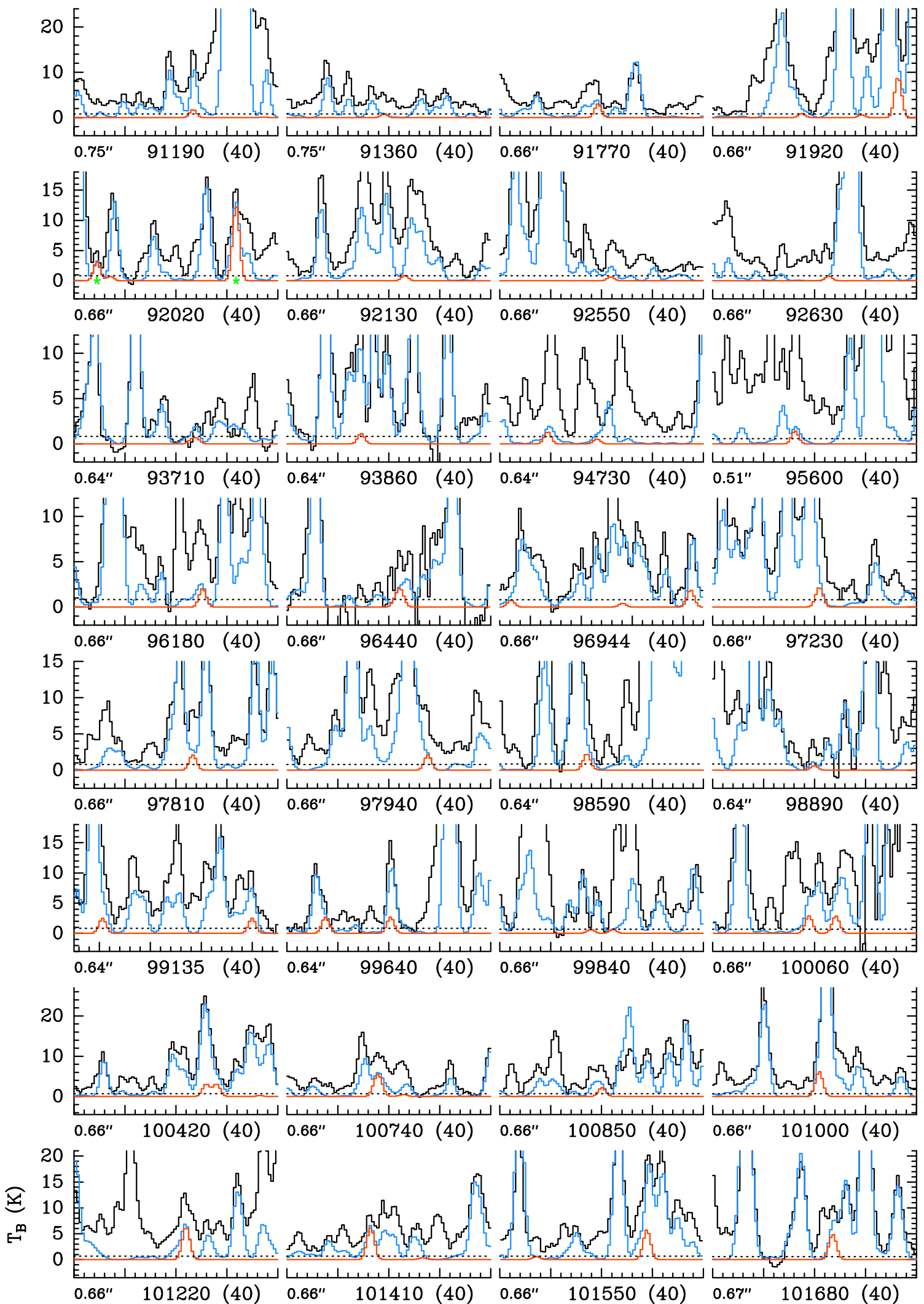

Fig. A.1. continued. 


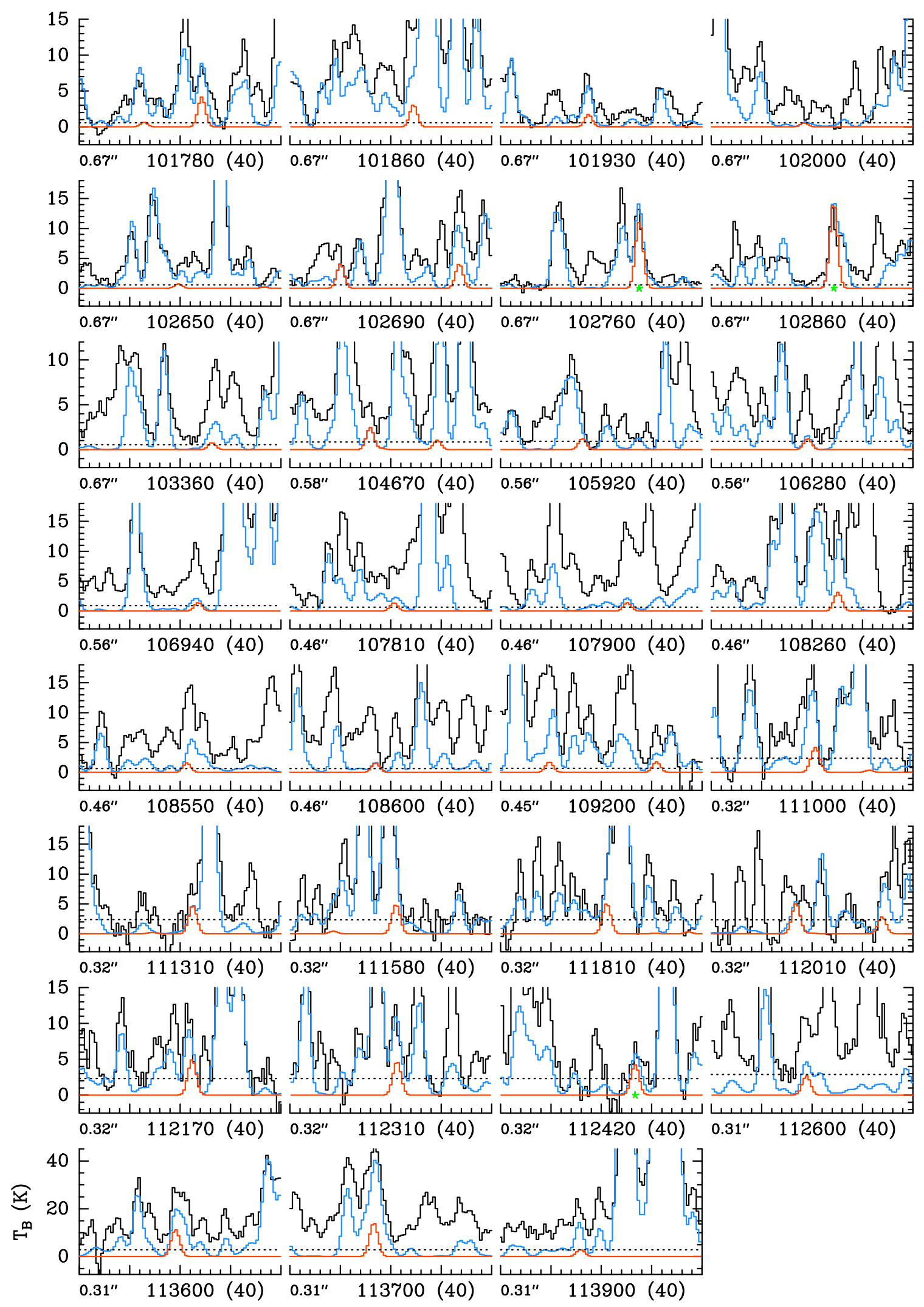

Fig. A.1. continued. 
A. Belloche et al.: Re-exploring molecular complexity with ALMA (ReMoCA): interstellar detection of urea
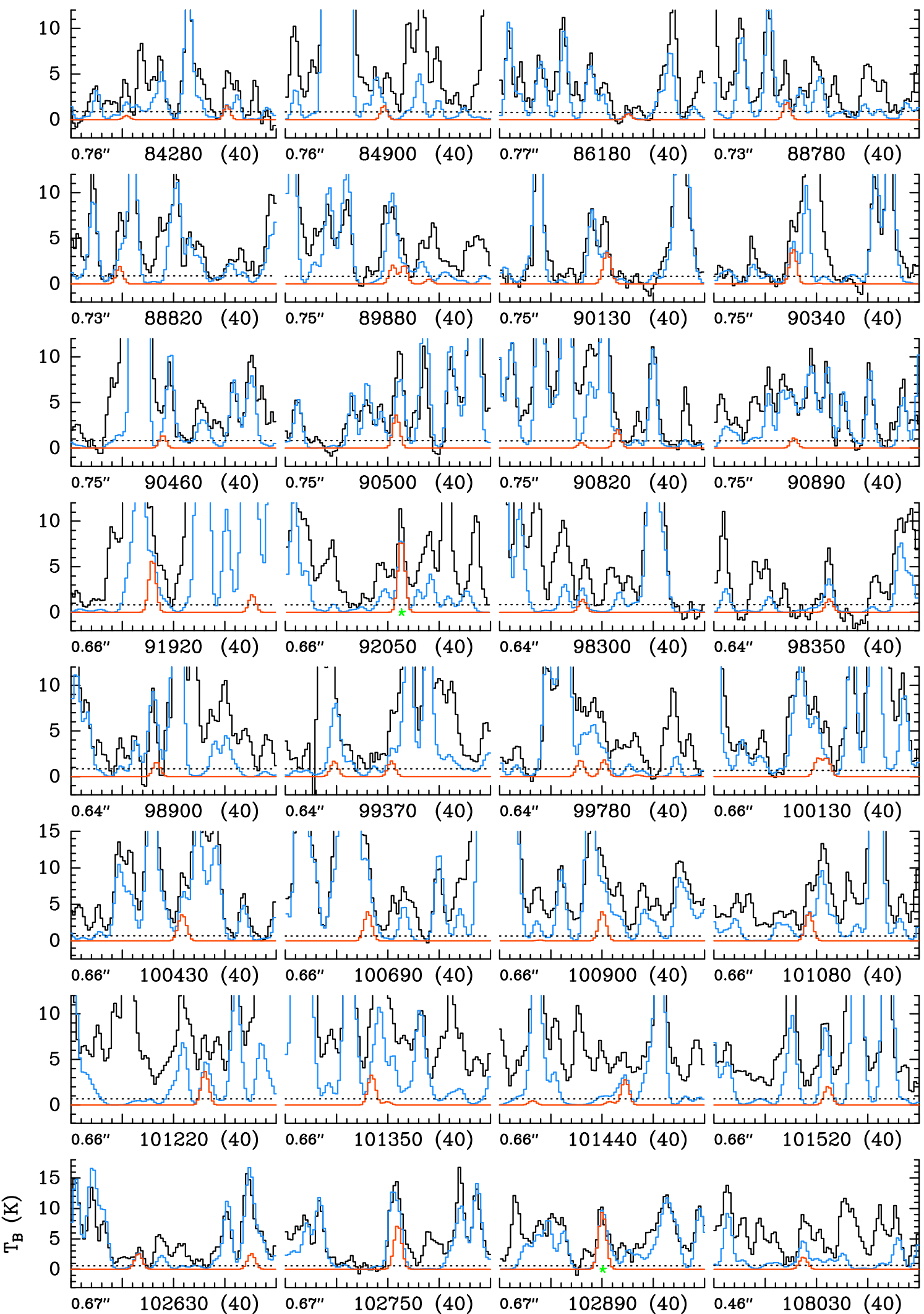

Fig. A.2. Same as Fig. A.1 for $\mathrm{NH}_{2} \mathrm{C}(\mathrm{O}) \mathrm{NH}_{2}, v=1$. 


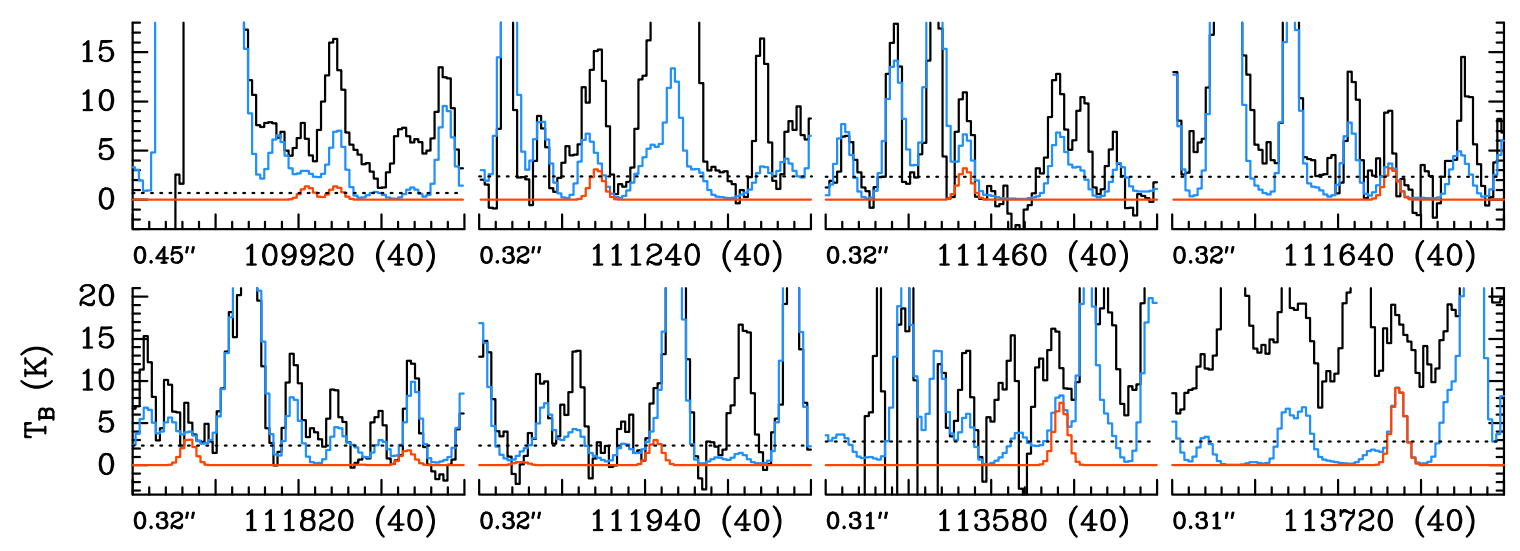

Fig. A.2. continued. 
A. Belloche et al.: Re-exploring molecular complexity with ALMA (ReMoCA): interstellar detection of urea
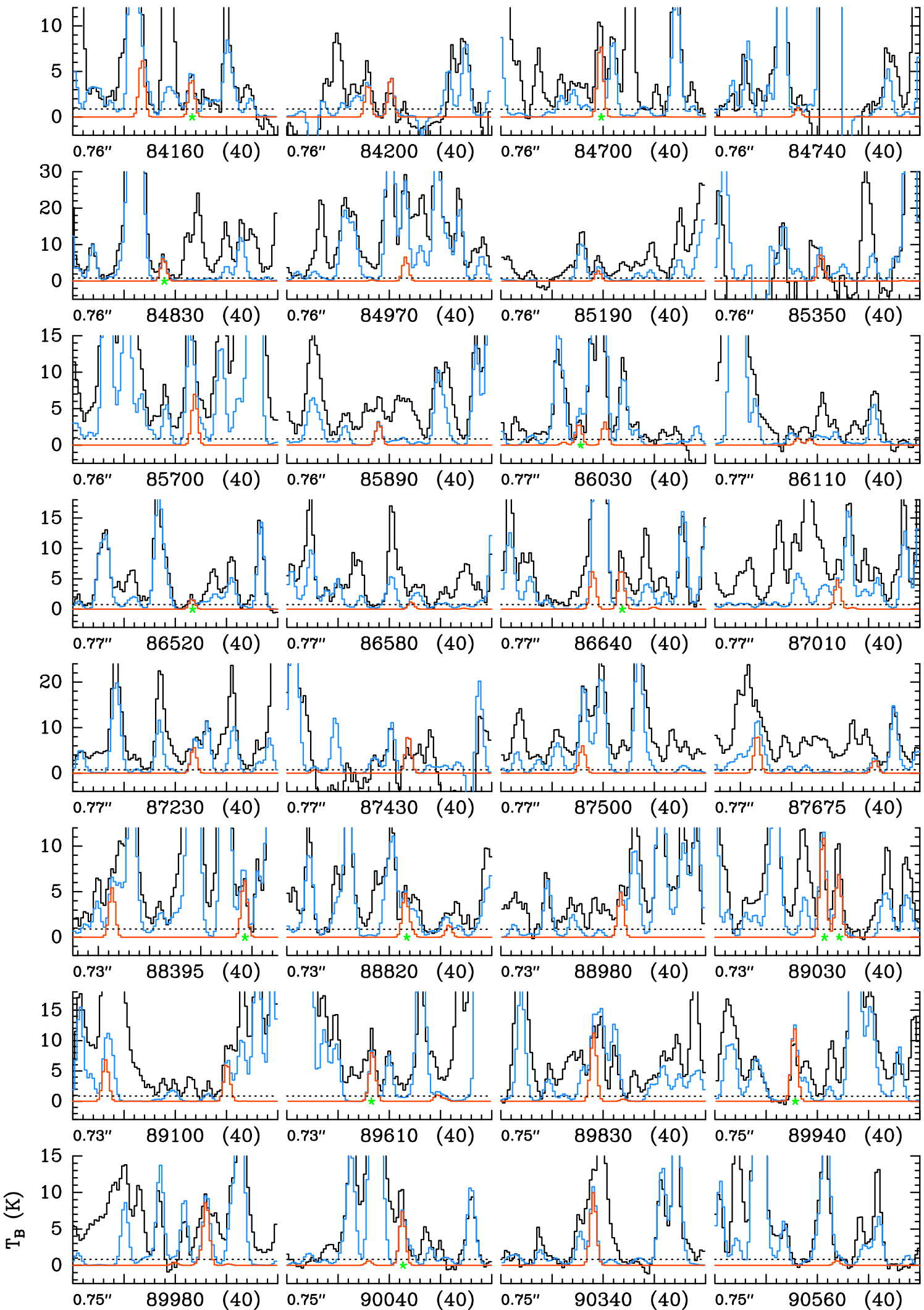

Fig. A.3. Same as Fig. A.1 for $\mathrm{CH}_{3} \mathrm{NHCHO}, v=0$. 


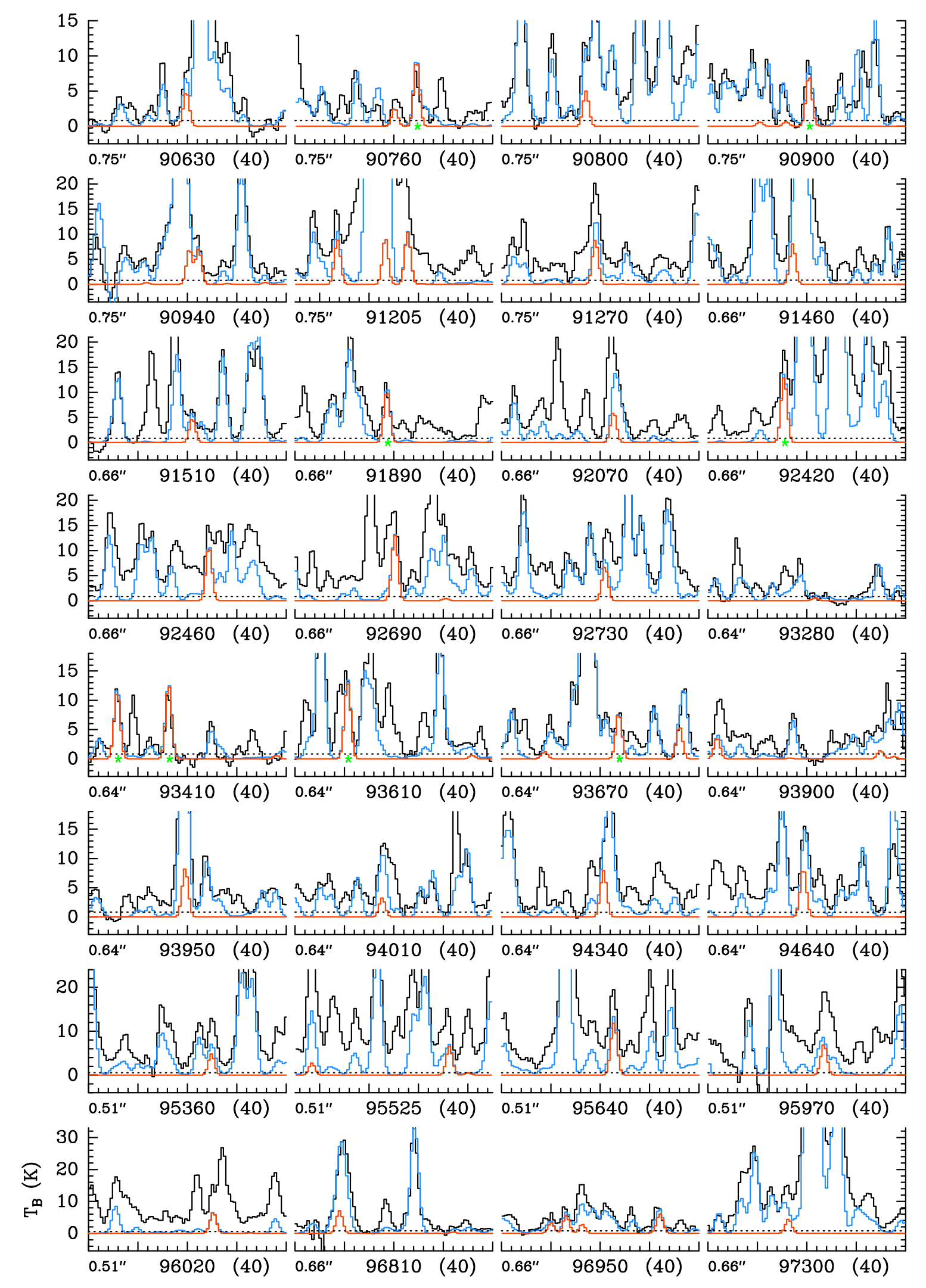

Fig. A.3. continued. 


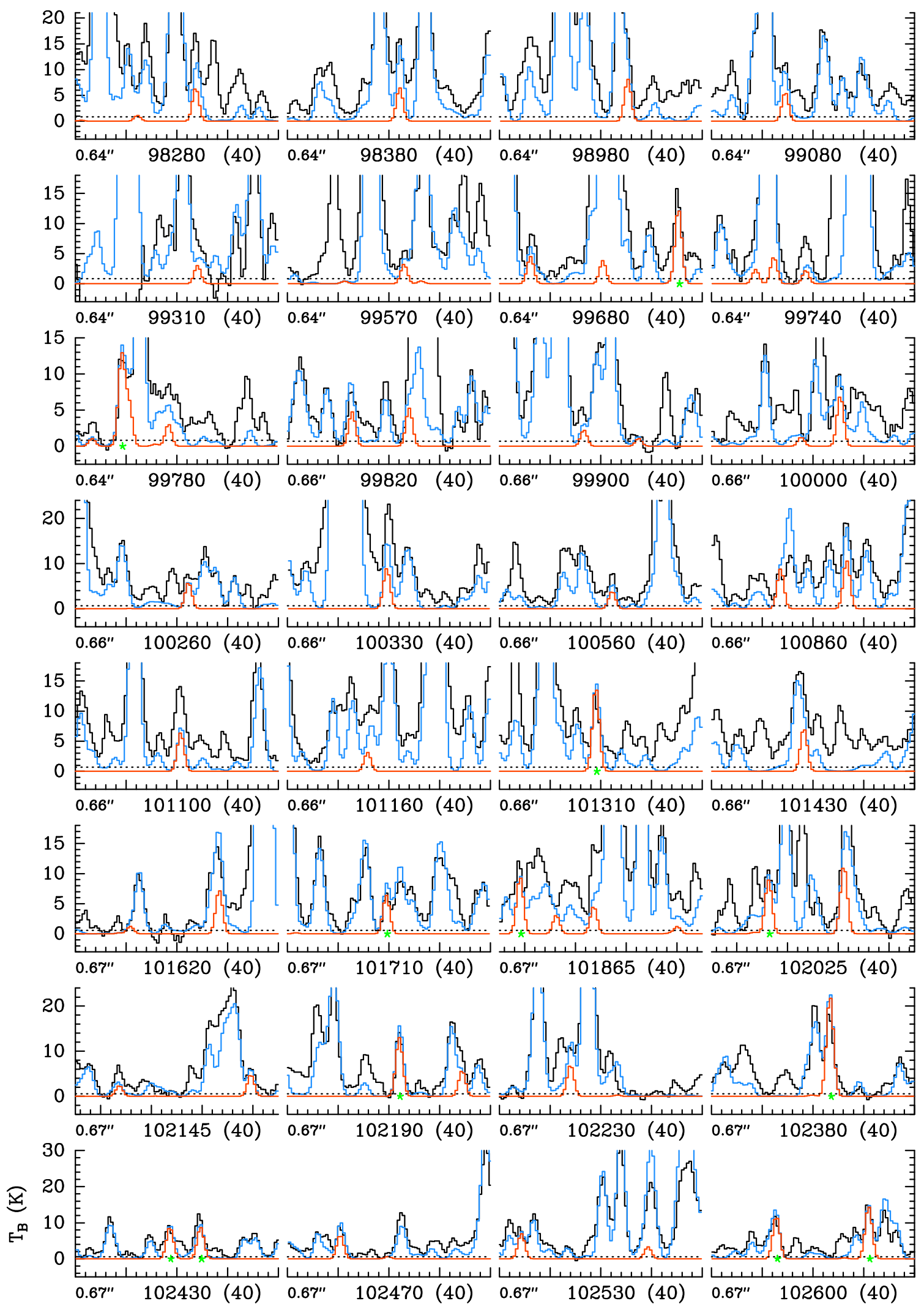

Fig. A.3. continued. 


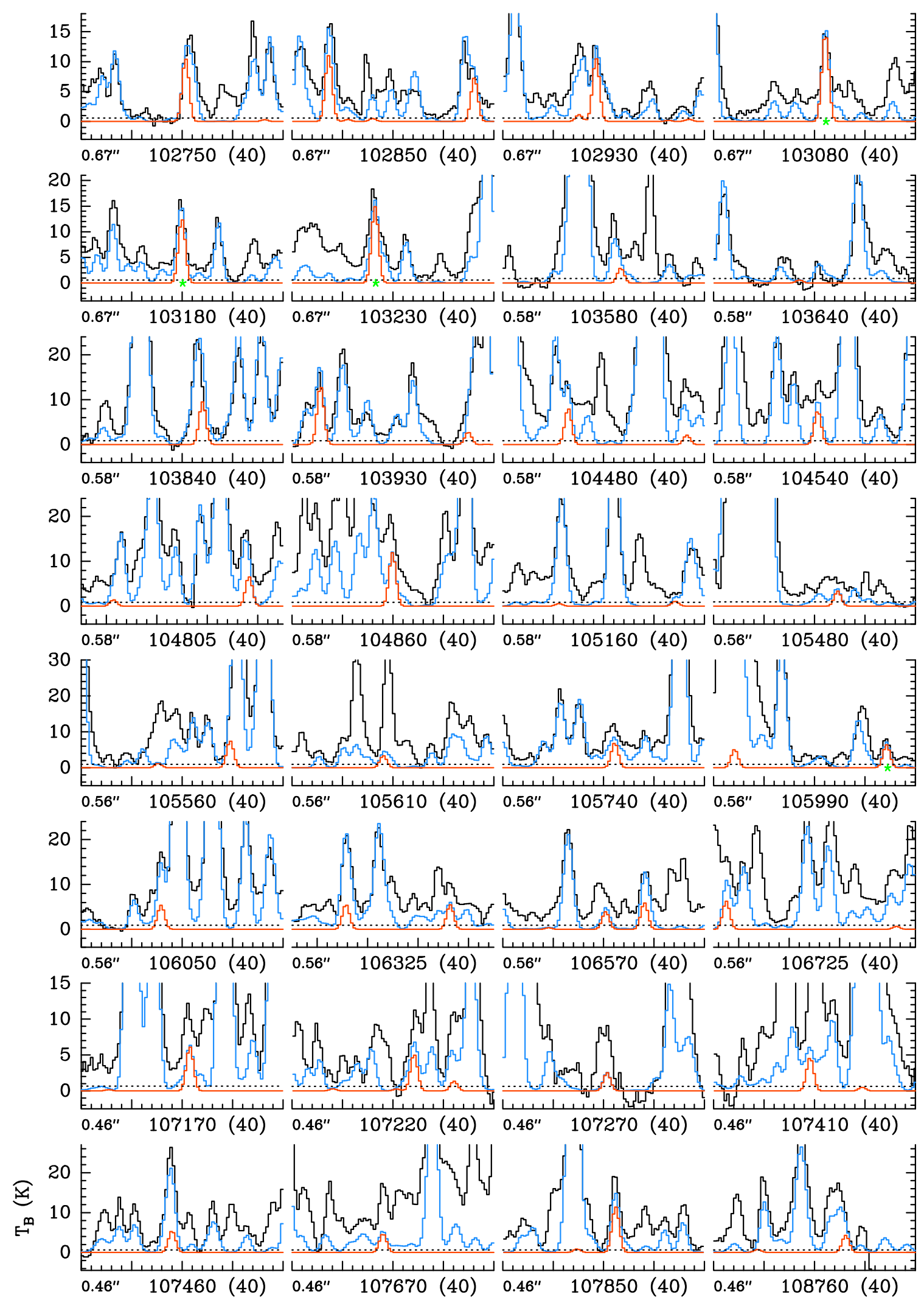

Fig. A.3. continued. 


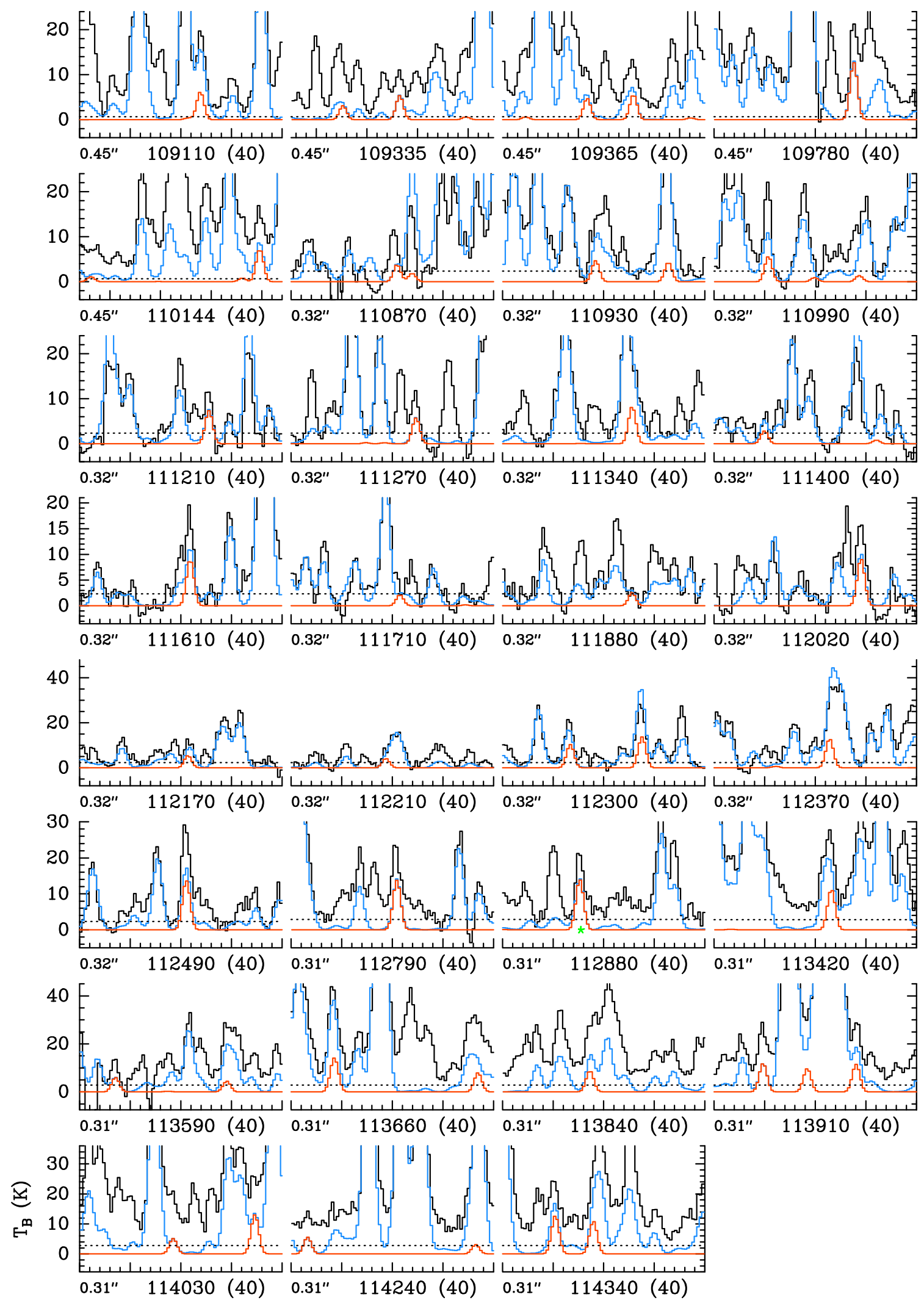

Fig. A.3. continued. 


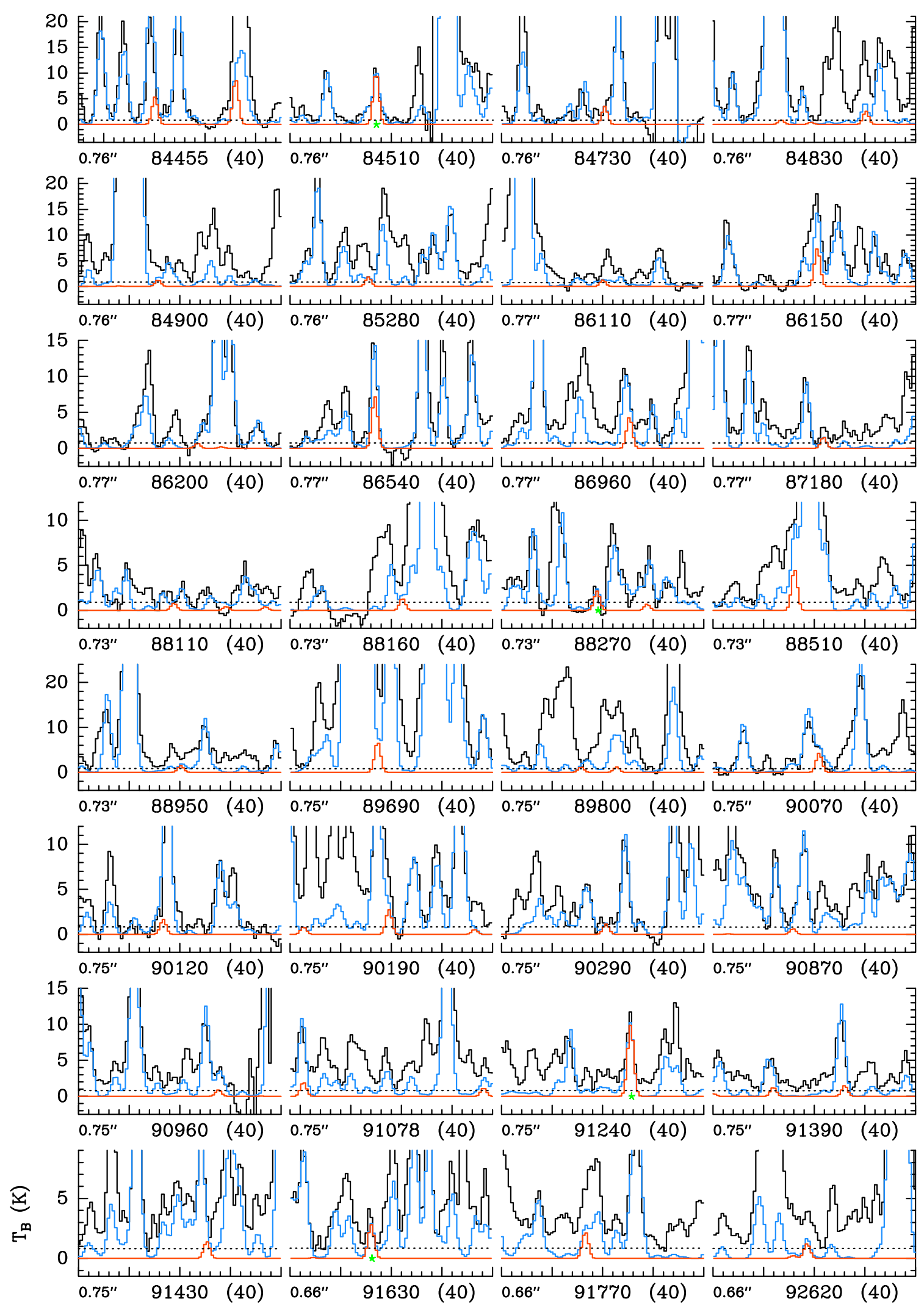

Fig. A.4. Same as Fig. A.1 for $\mathrm{CH}_{3} \mathrm{NHCHO}, v_{\mathrm{t}}=1$. 
A. Belloche et al.: Re-exploring molecular complexity with ALMA (ReMoCA): interstellar detection of urea
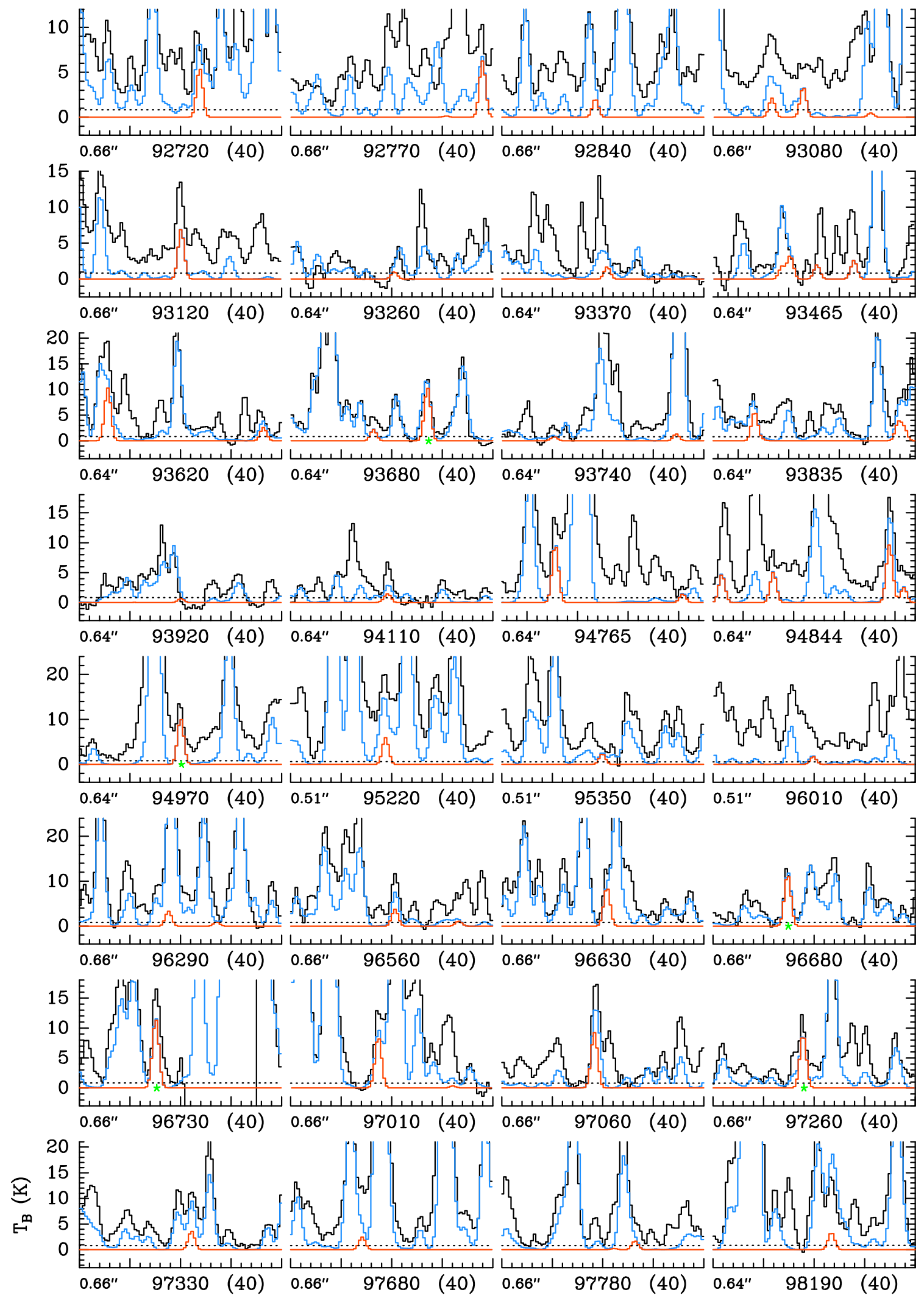

Fig. A.4. continued. 

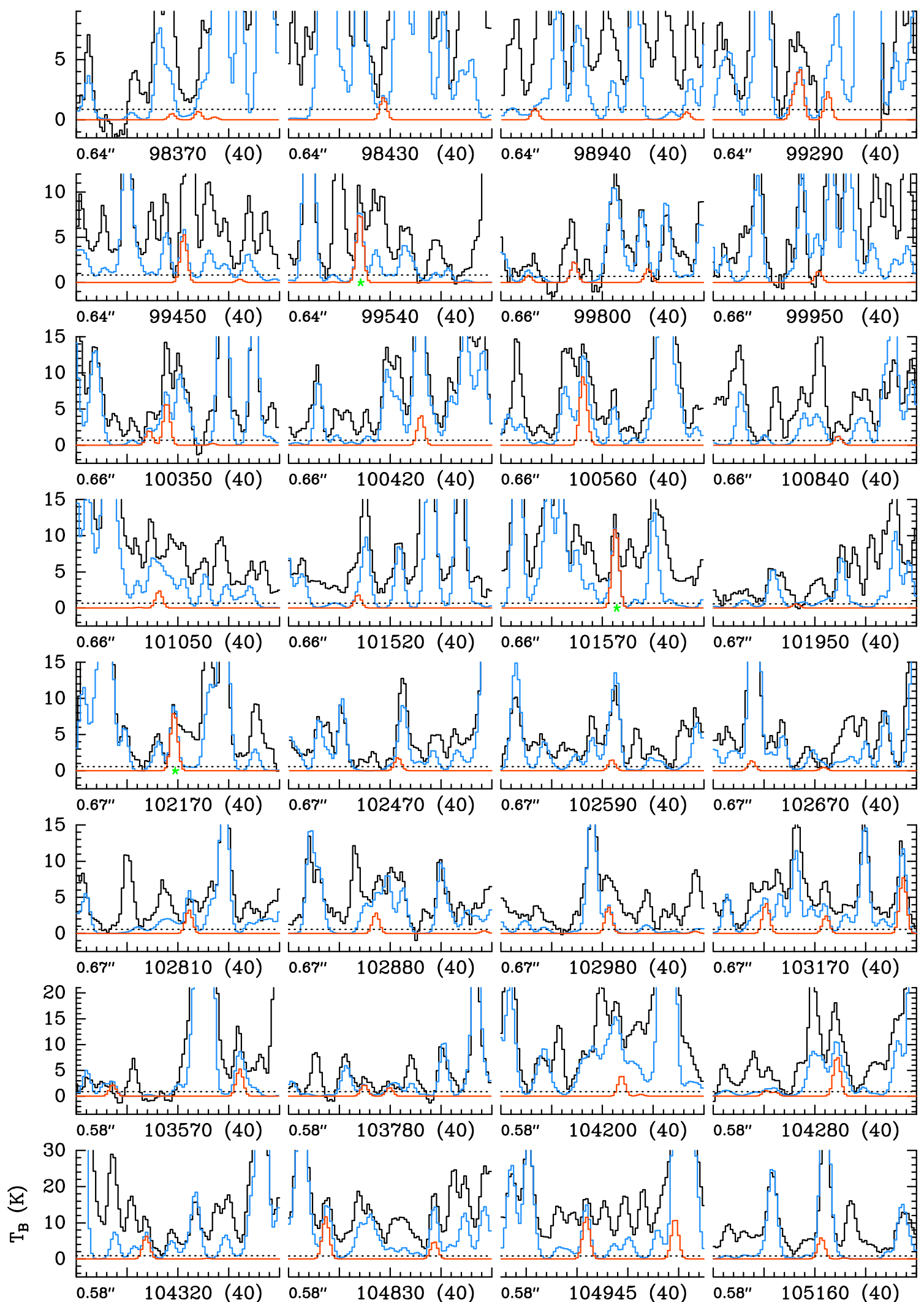

Fig. A.4. continued. 


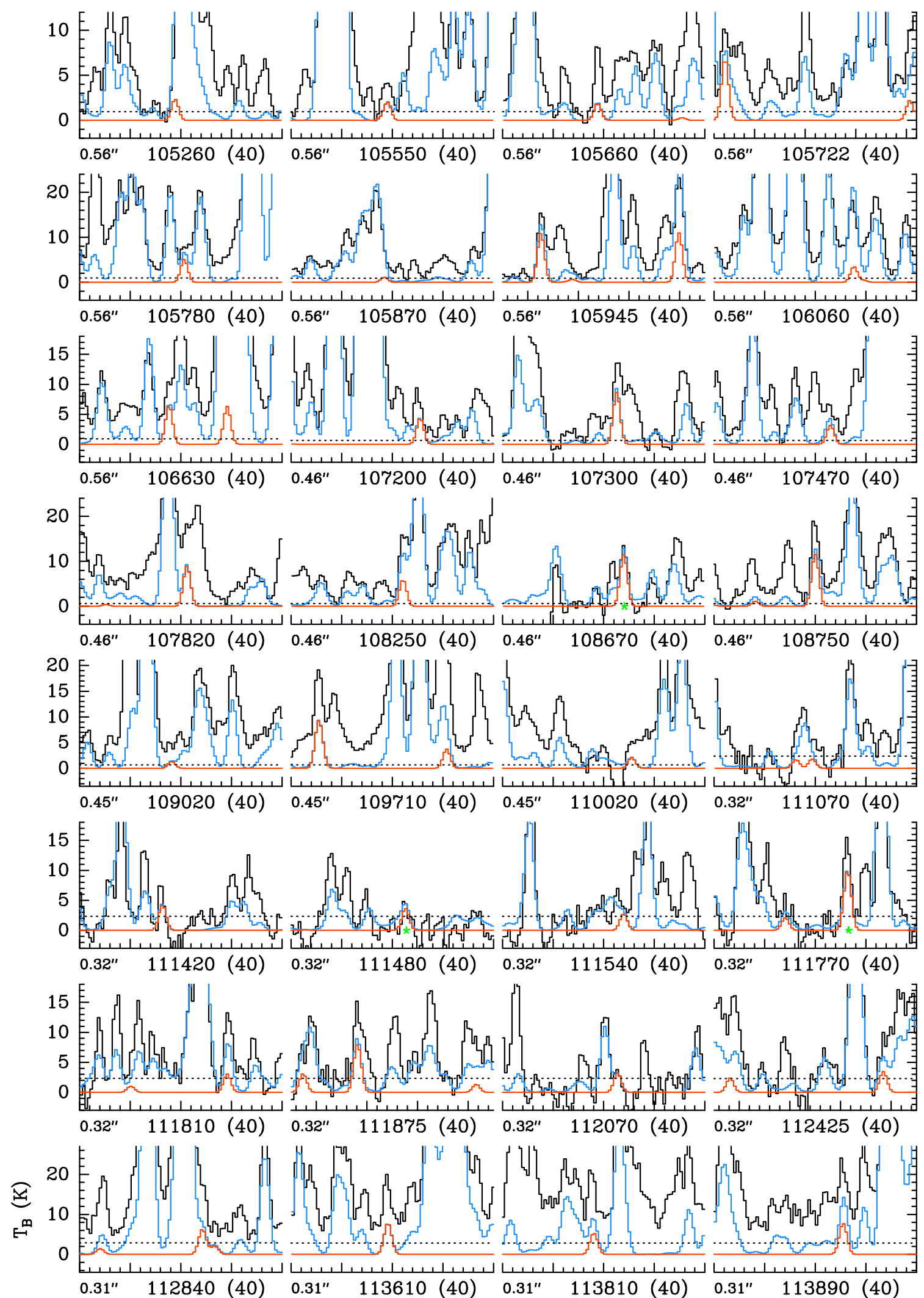

Fig. A.4. continued. 


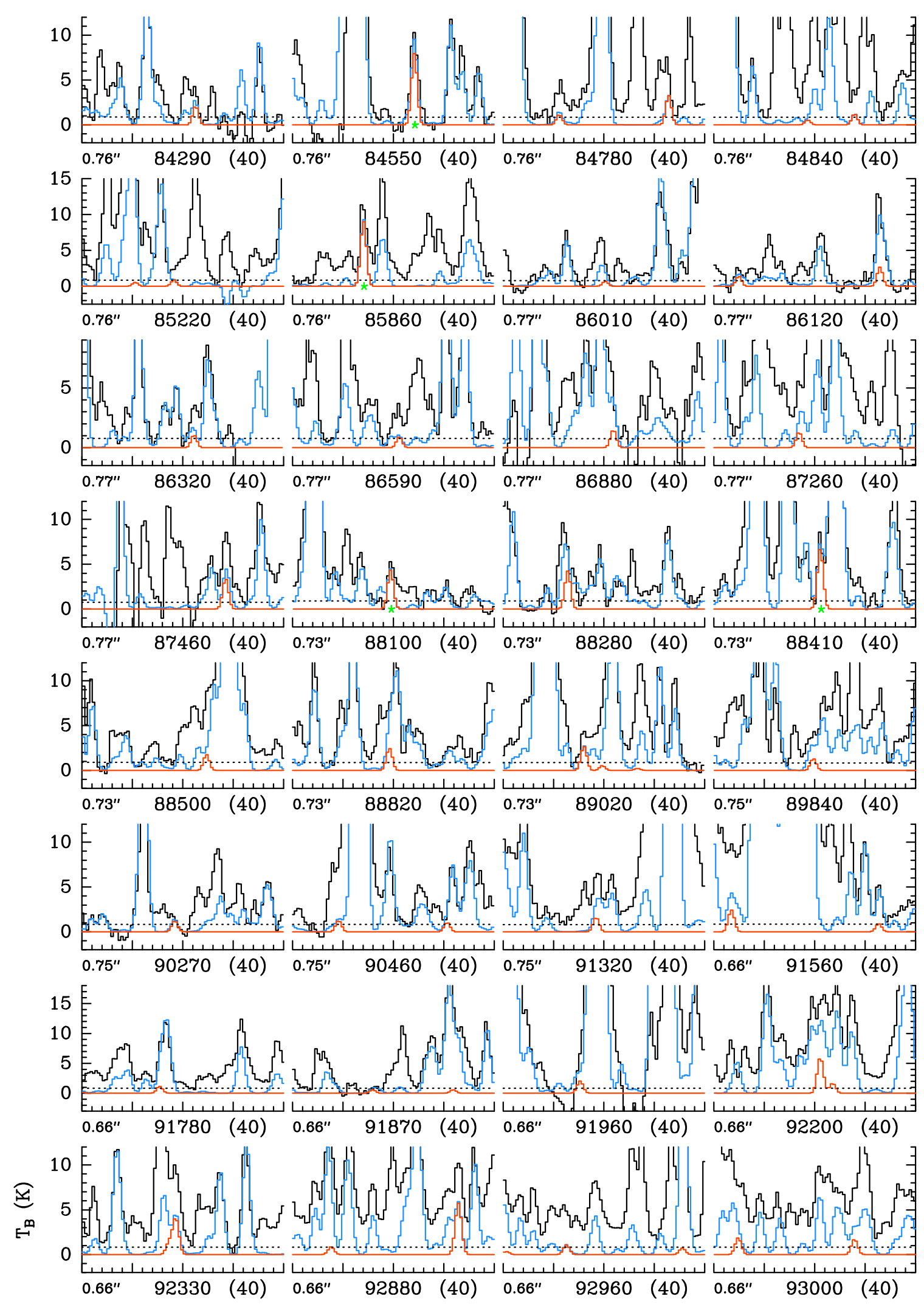

Fig. A.5. Same as Fig. A.1 for $\mathrm{CH}_{3} \mathrm{NHCHO}, v_{\mathrm{t}}=2$. 
A. Belloche et al.: Re-exploring molecular complexity with ALMA (ReMoCA): interstellar detection of urea

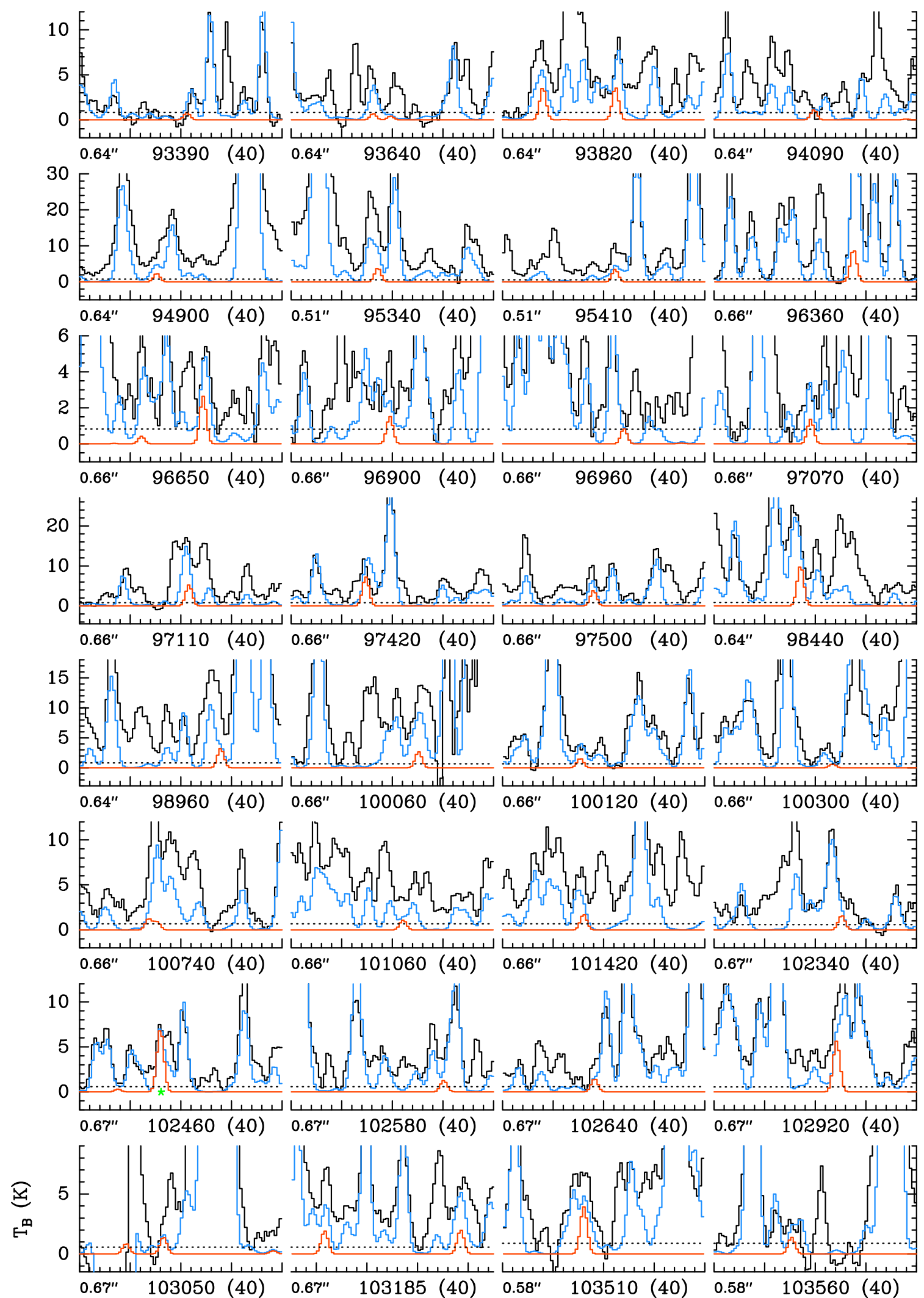

Fig. A.5. continued. 


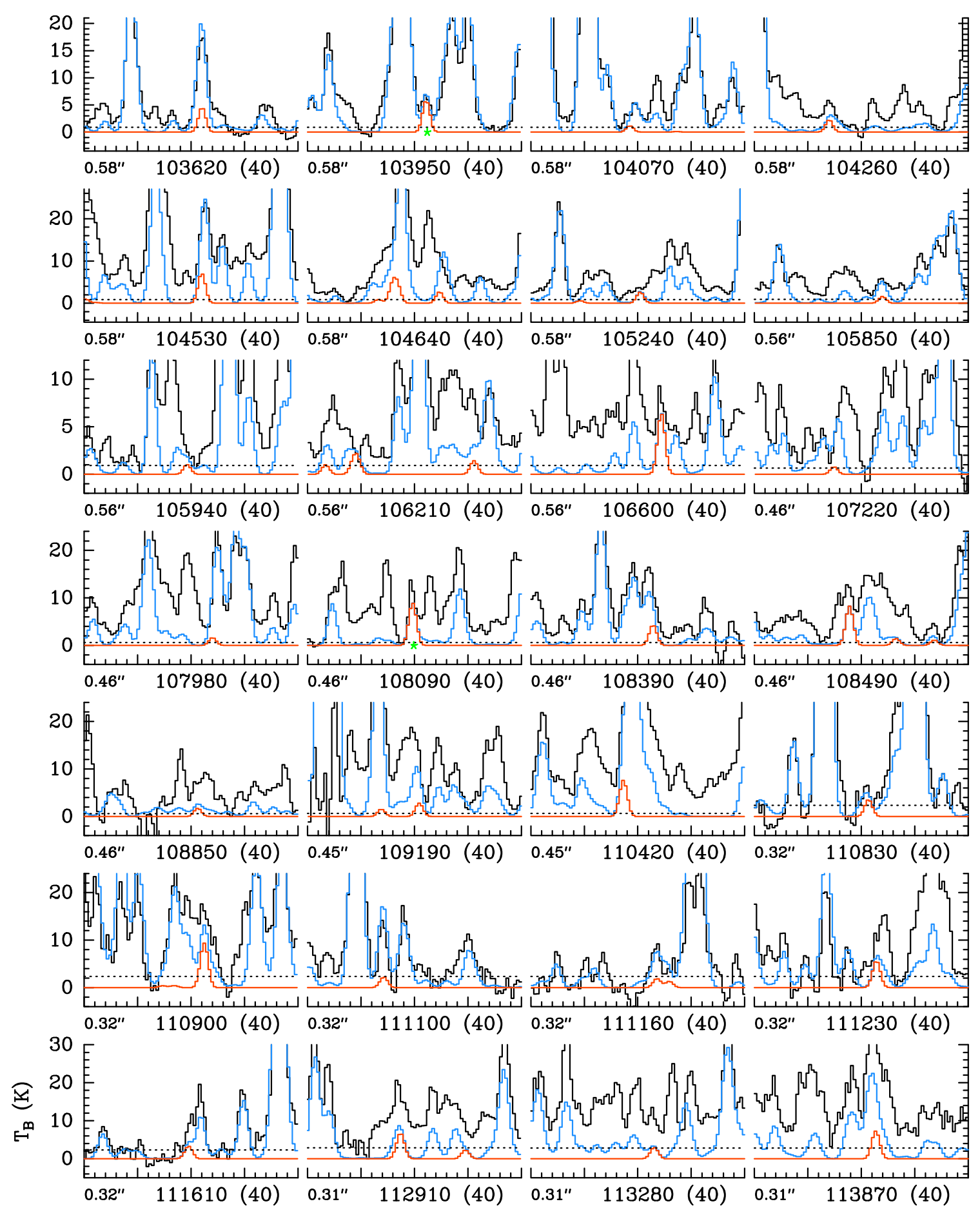

Fig. A.5. continued. 

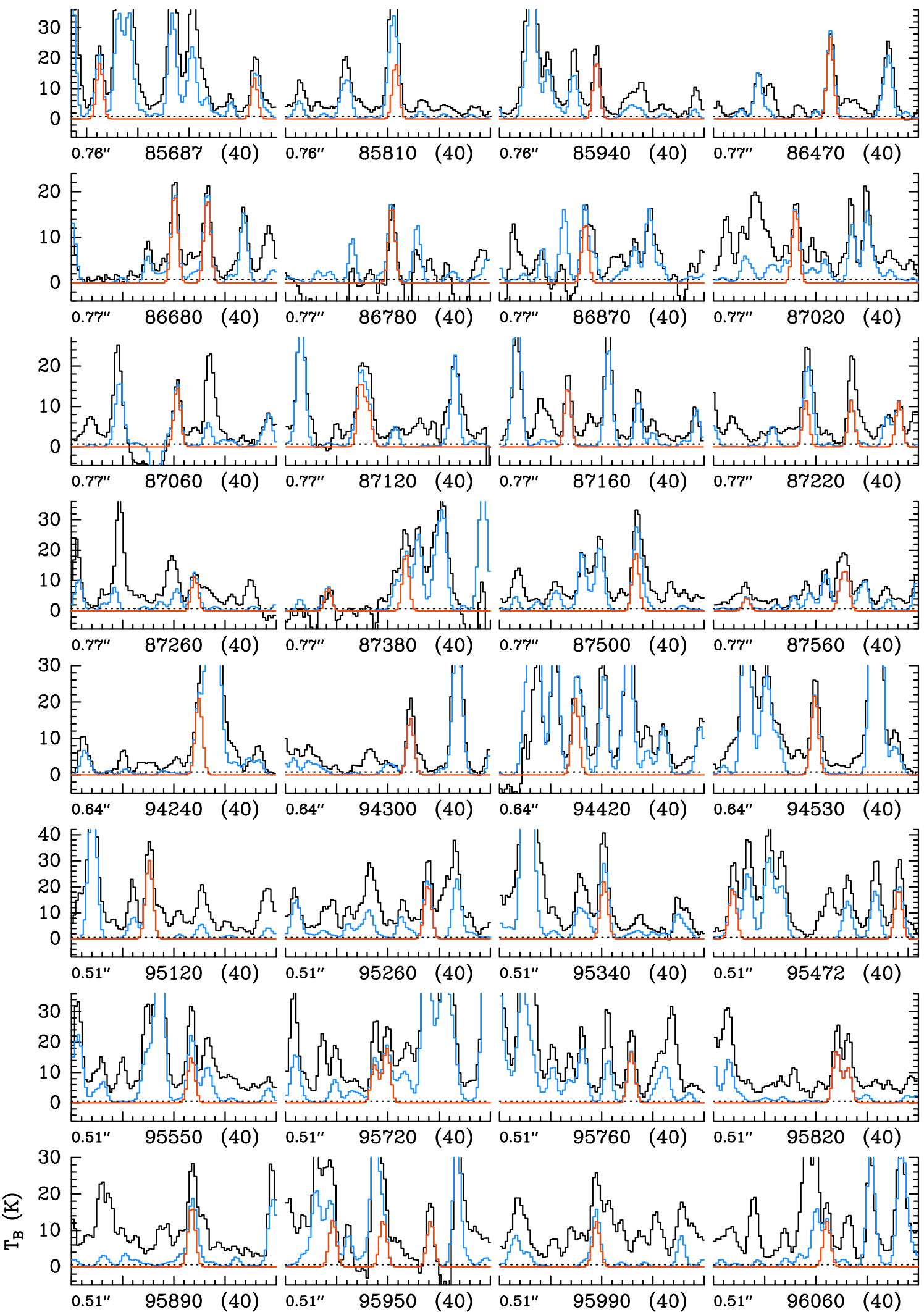

Fig. A.6. Same as Fig. A.1 for $\mathrm{CH}_{3} \mathrm{NCO}, v=0$. 


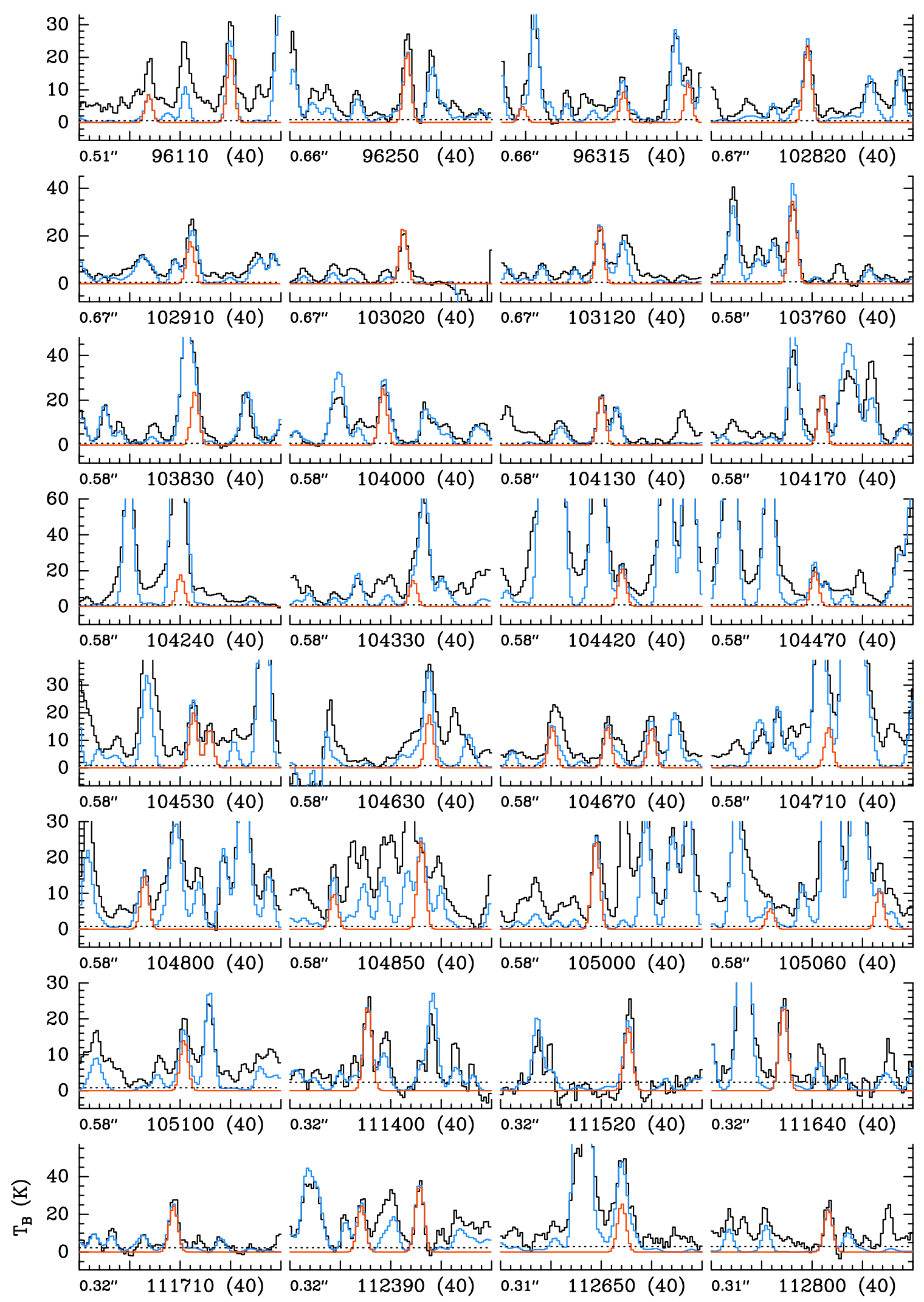

Fig. A.6. continued. 
A. Belloche et al.: Re-exploring molecular complexity with ALMA (ReMoCA): interstellar detection of urea
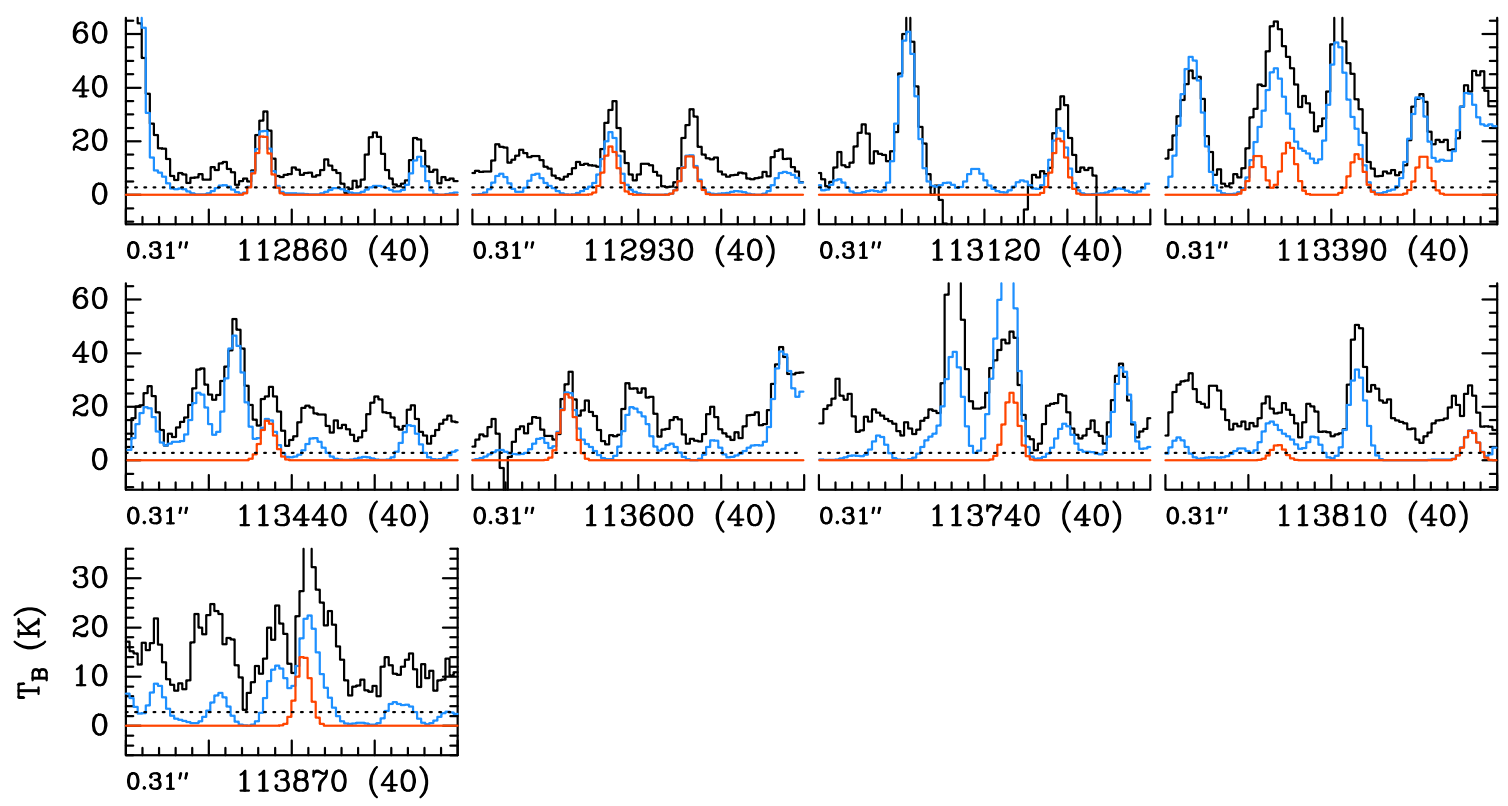

Fig. A.6. continued.

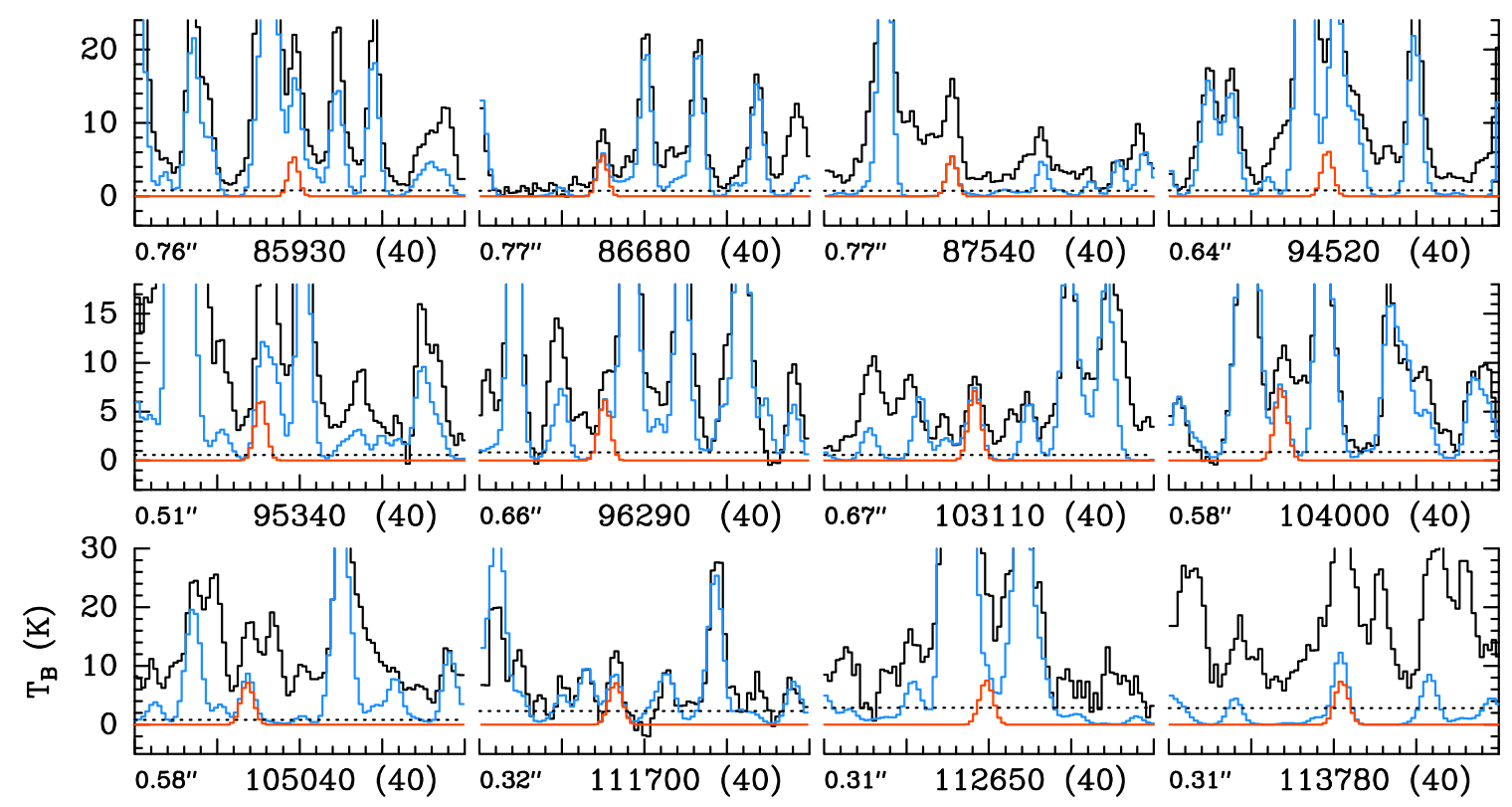

Fig. A.7. Same as Fig. A.1 for $\mathrm{CH}_{3} \mathrm{NCO}, v_{\mathrm{b}}=1$. 

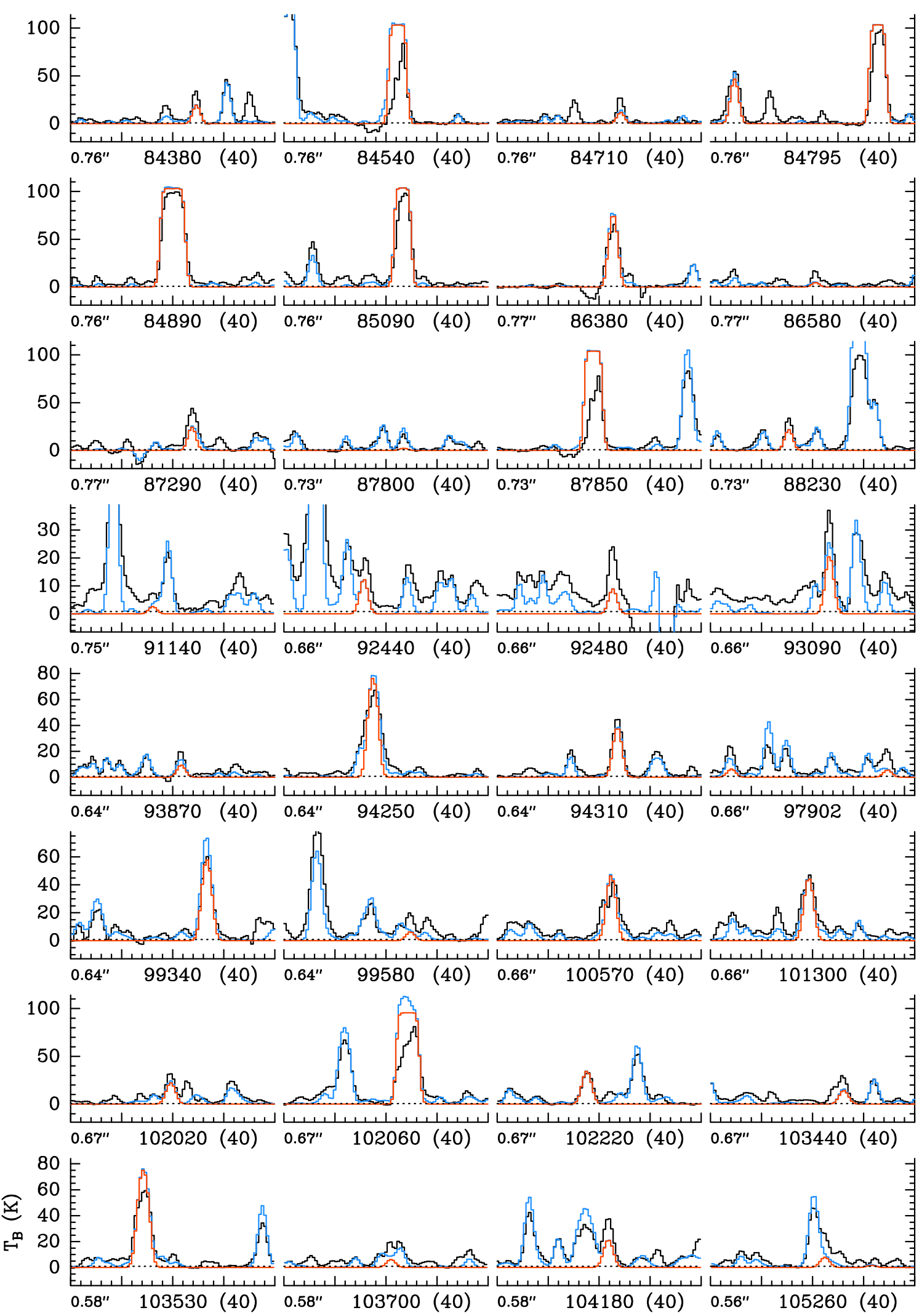

Fig. A.8. Same as Fig. A.1 for $\mathrm{NH}_{2} \mathrm{CHO}, v=0$. 
A. Belloche et al.: Re-exploring molecular complexity with ALMA (ReMoCA): interstellar detection of urea
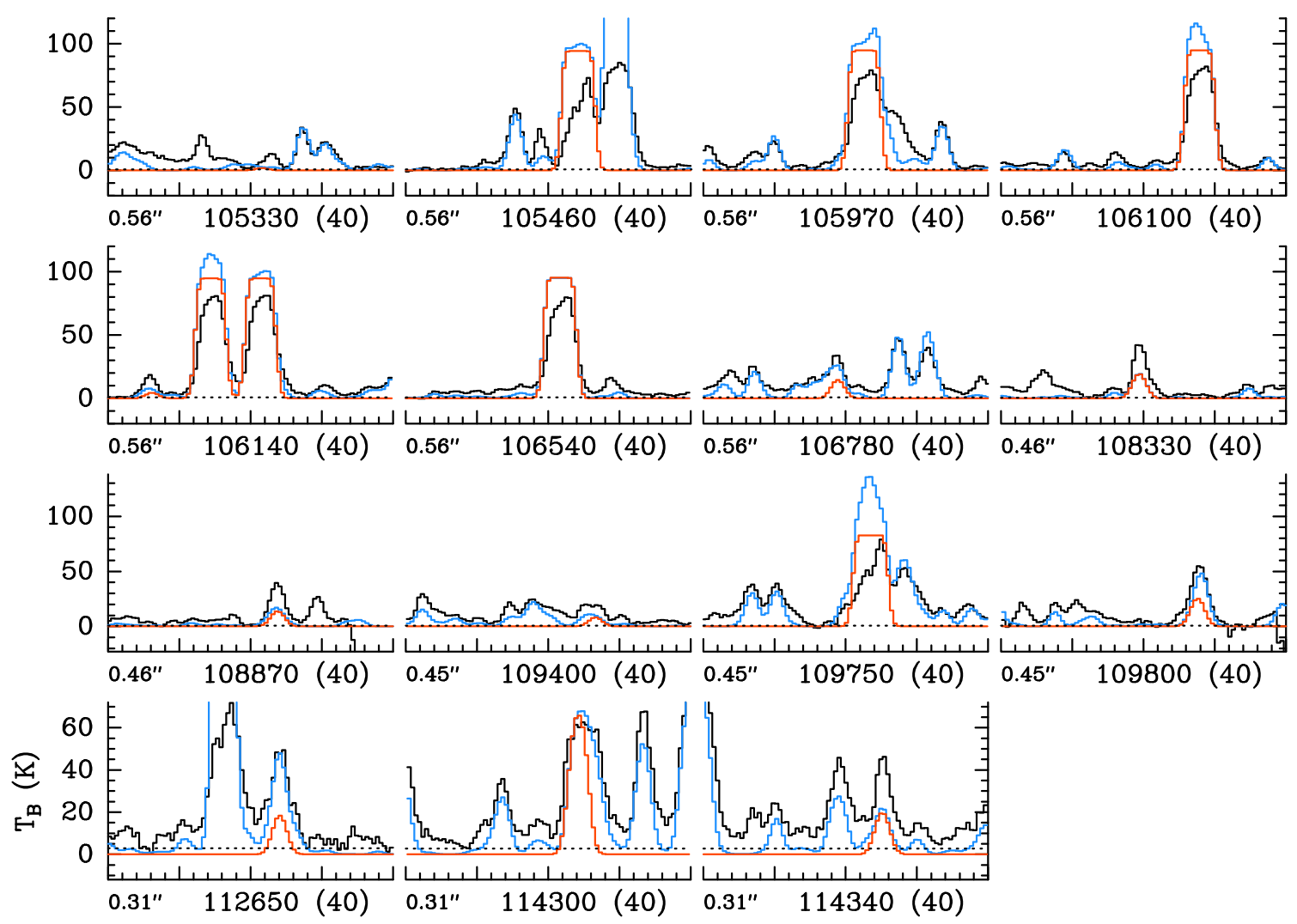

Fig. A.8. continued. 


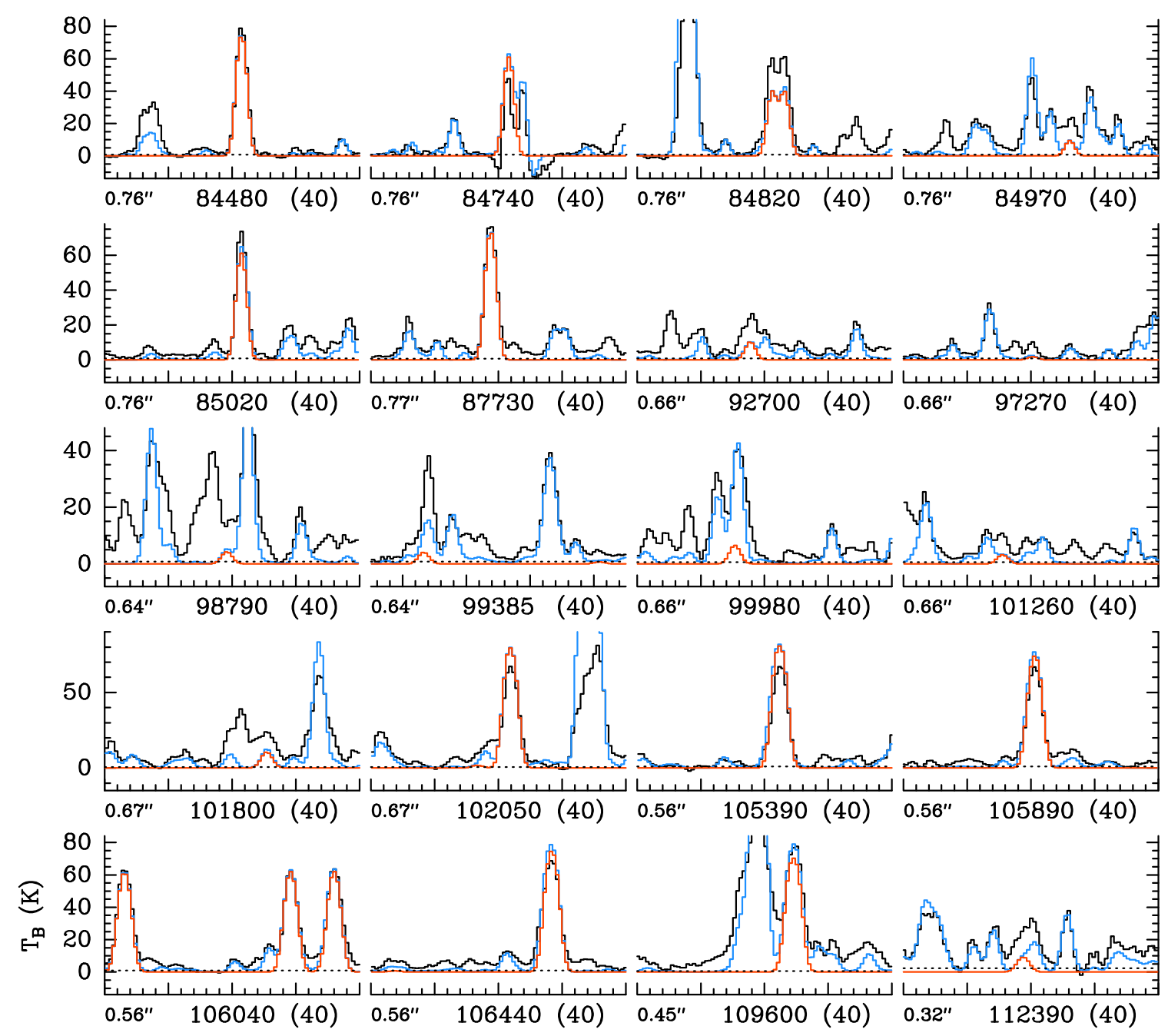

Fig. A.9. Same as Fig. A.1 for $\mathrm{NH}_{2} \mathrm{CHO}, v_{12}=1$. 
A. Belloche et al.: Re-exploring molecular complexity with ALMA (ReMoCA): interstellar detection of urea
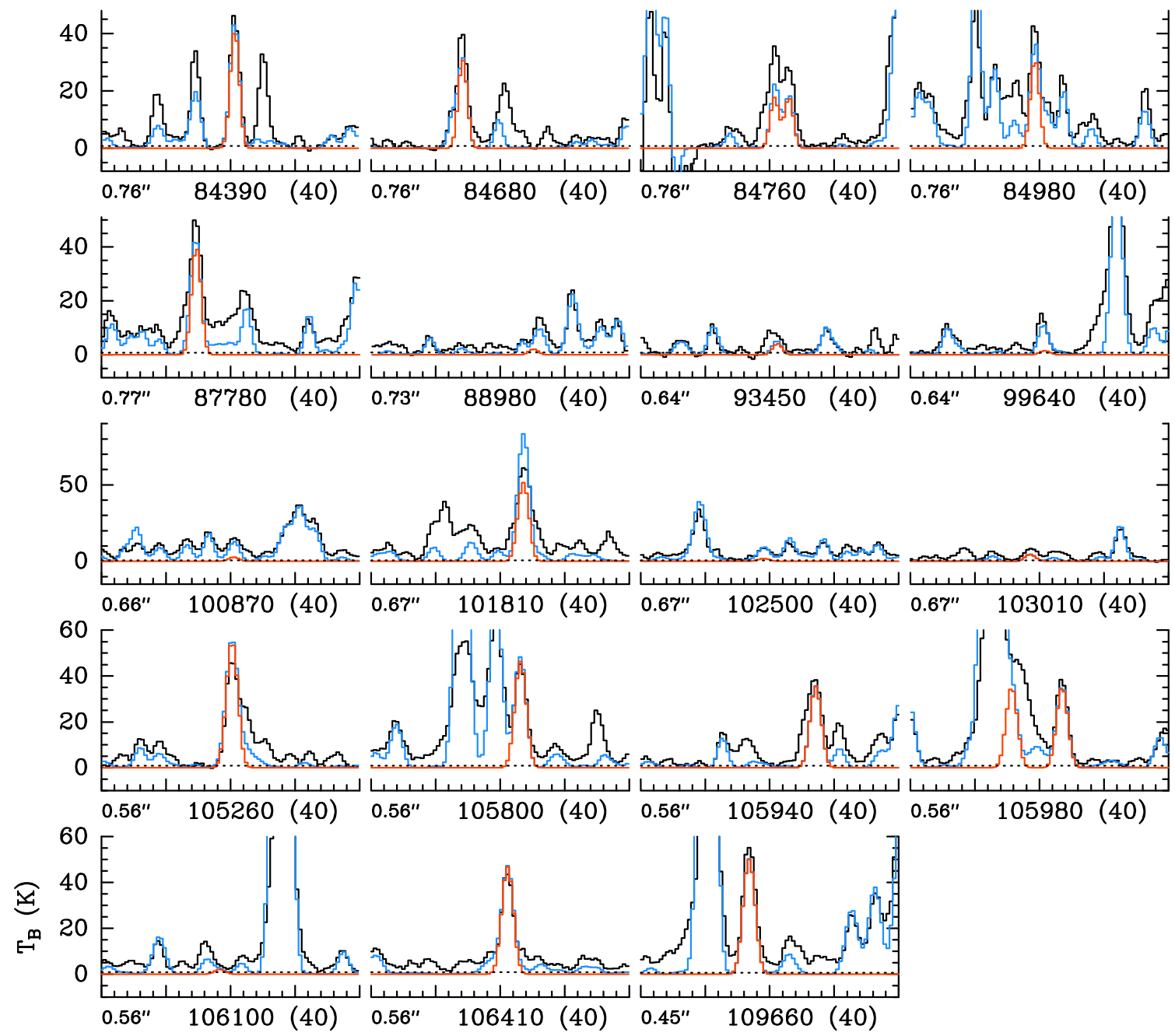

Fig. A.10. Same as Fig. A.1 for $\mathrm{NH}_{2}{ }^{13} \mathrm{CHO}, v=0$.
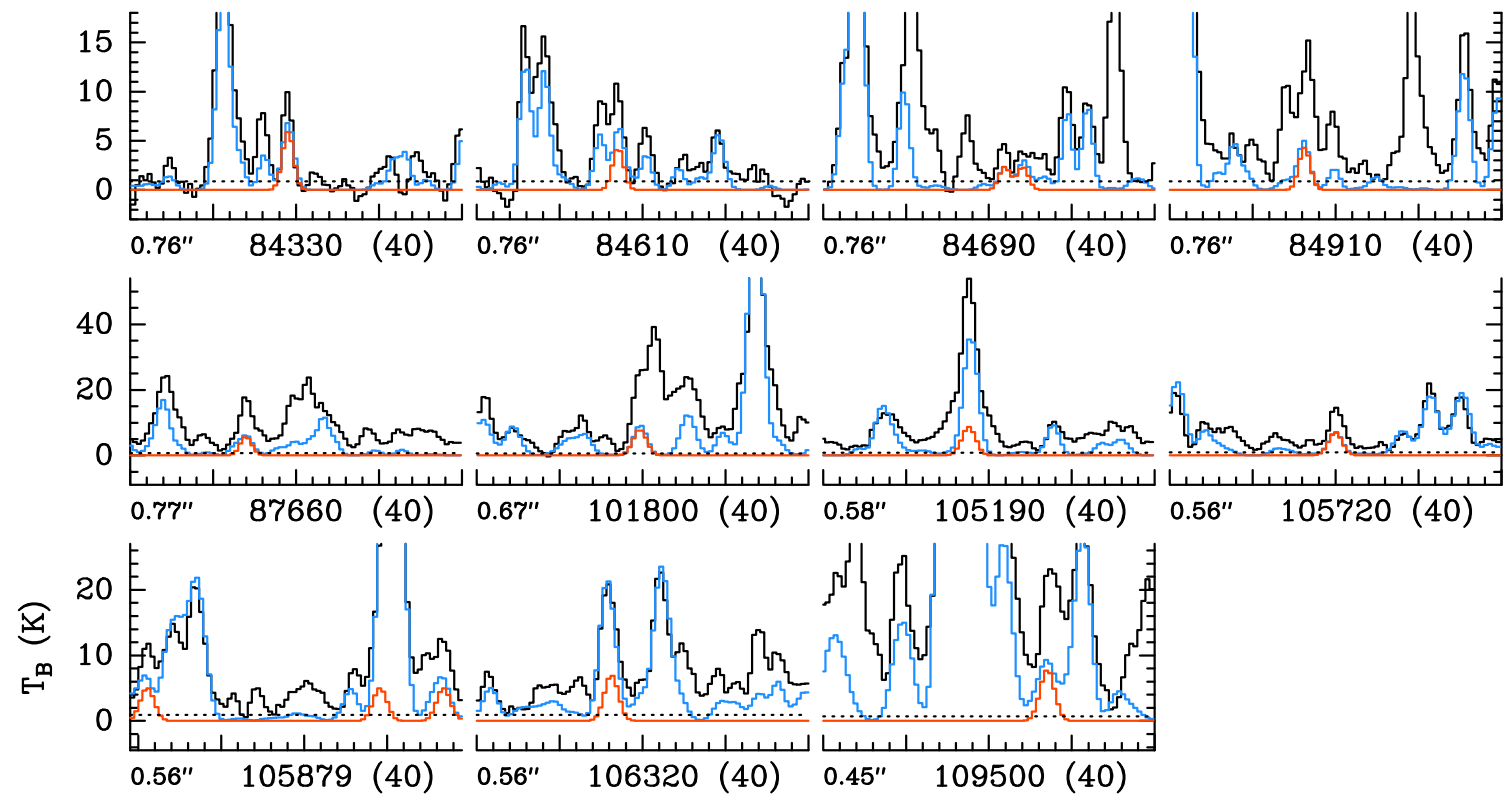

Fig. A.11. Same as Fig. A. 1 for $\mathrm{NH}_{2}{ }^{13} \mathrm{CHO}, v_{12}=1$. 


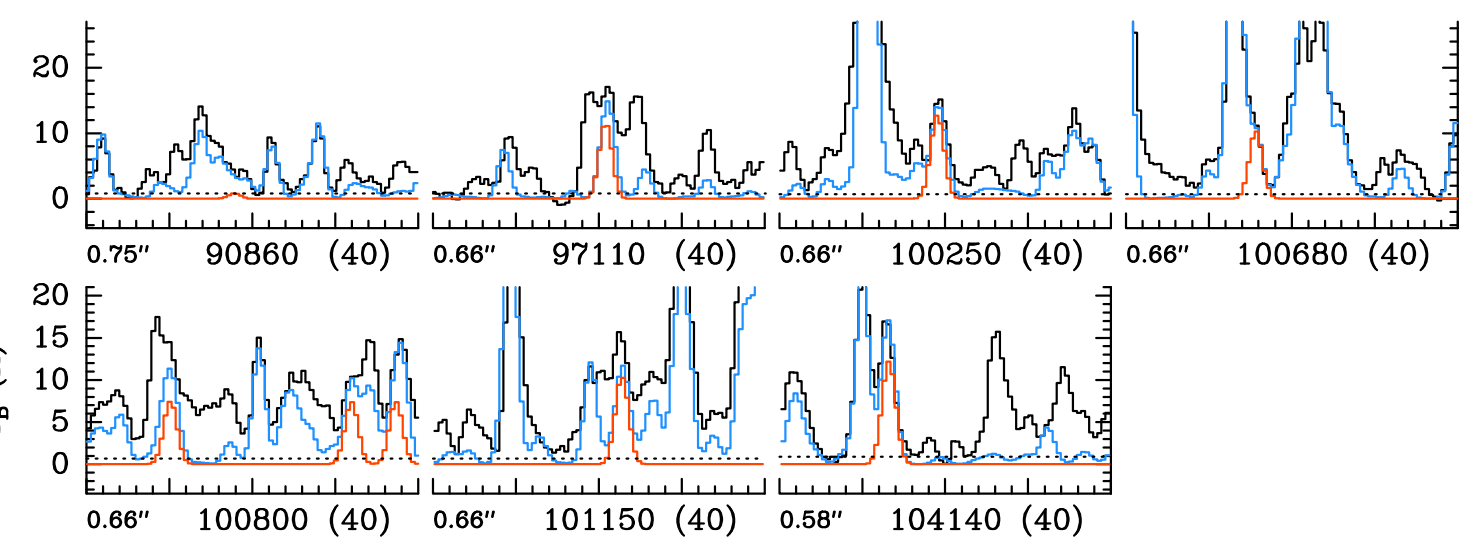

Fig. A.12. Same as Fig. A.1 for $\mathrm{NH}_{2} \mathrm{CH}^{18} \mathrm{O}, v=0$.

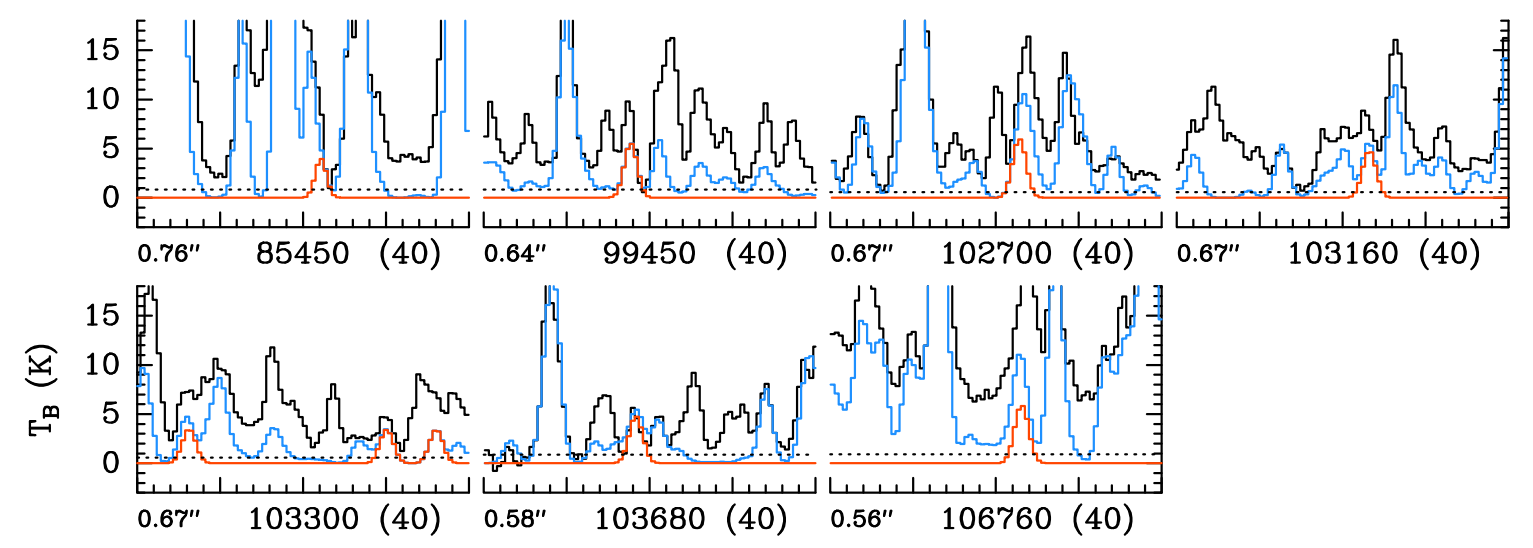

Fig. A.13. Same as Fig. A.1 for ${ }^{15} \mathrm{NH}_{2} \mathrm{CHO}, v=0$. 
A. Belloche et al.: Re-exploring molecular complexity with ALMA (ReMoCA): interstellar detection of urea
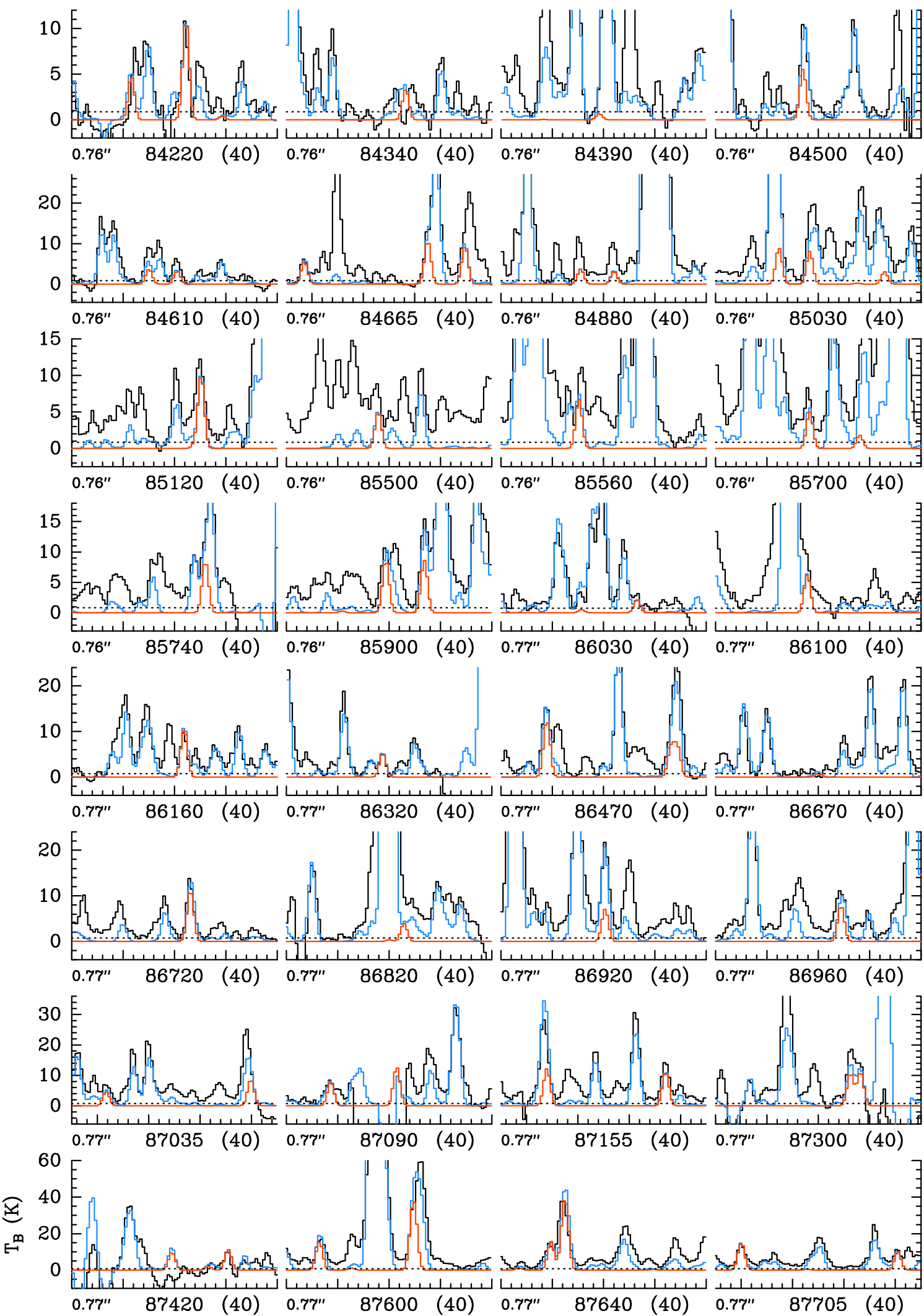

Fig. A.14. Same as Fig. A.1 for $\mathrm{CH}_{3} \mathrm{C}(\mathrm{O}) \mathrm{NH}_{2}, v=0$. 


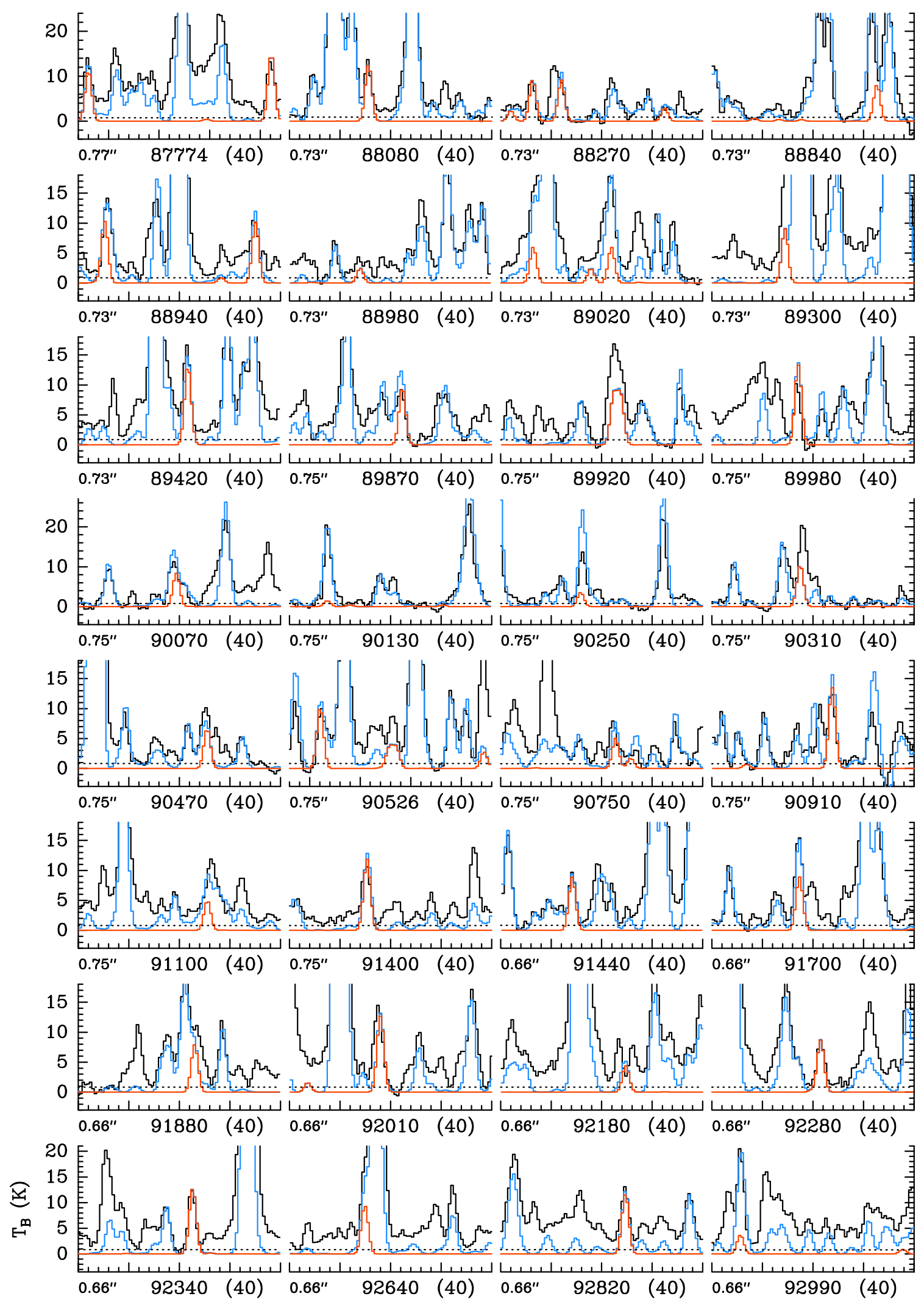

Fig. A.14. continued. 
A. Belloche et al.: Re-exploring molecular complexity with ALMA (ReMoCA): interstellar detection of urea
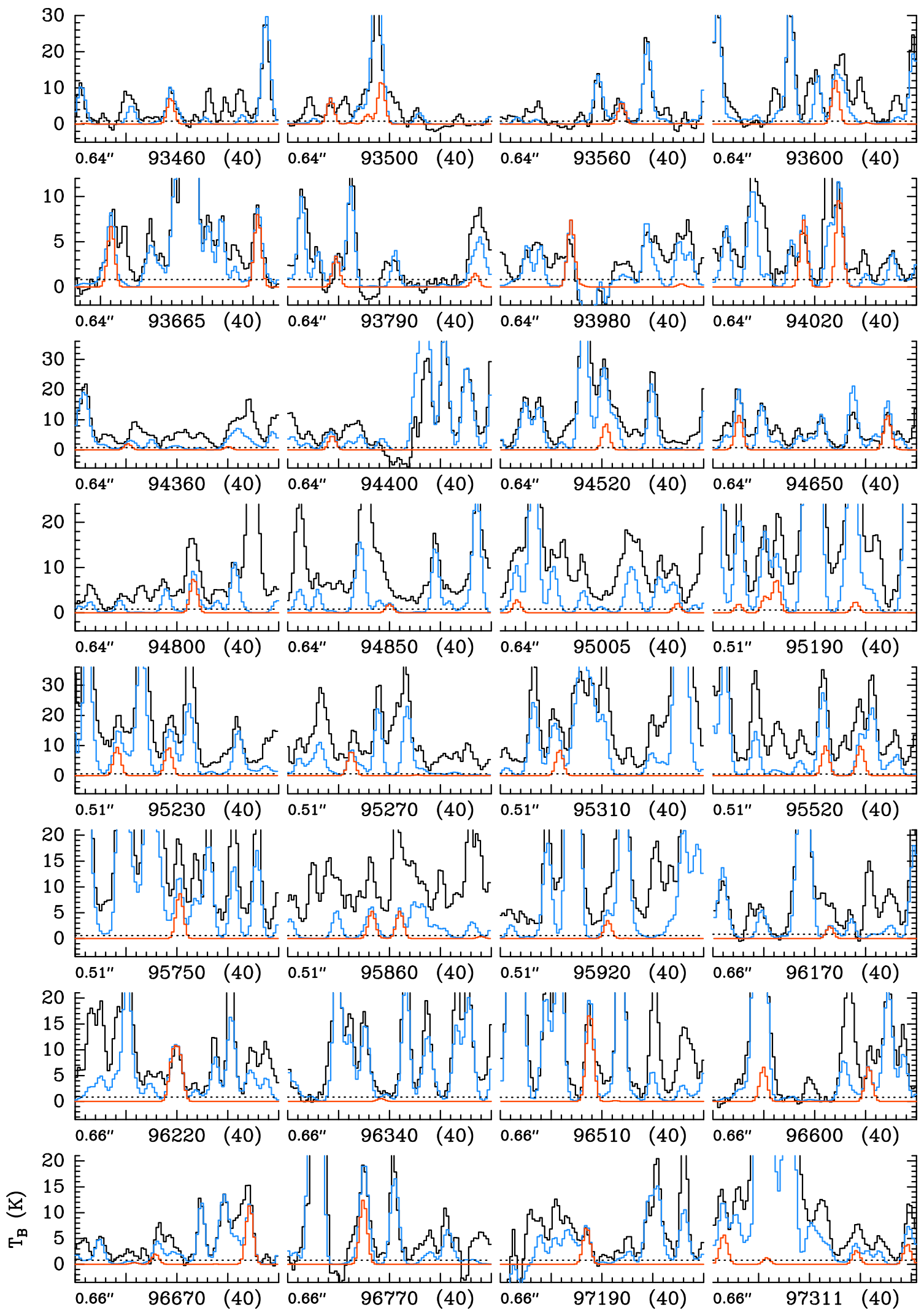

Fig. A.14. continued. 


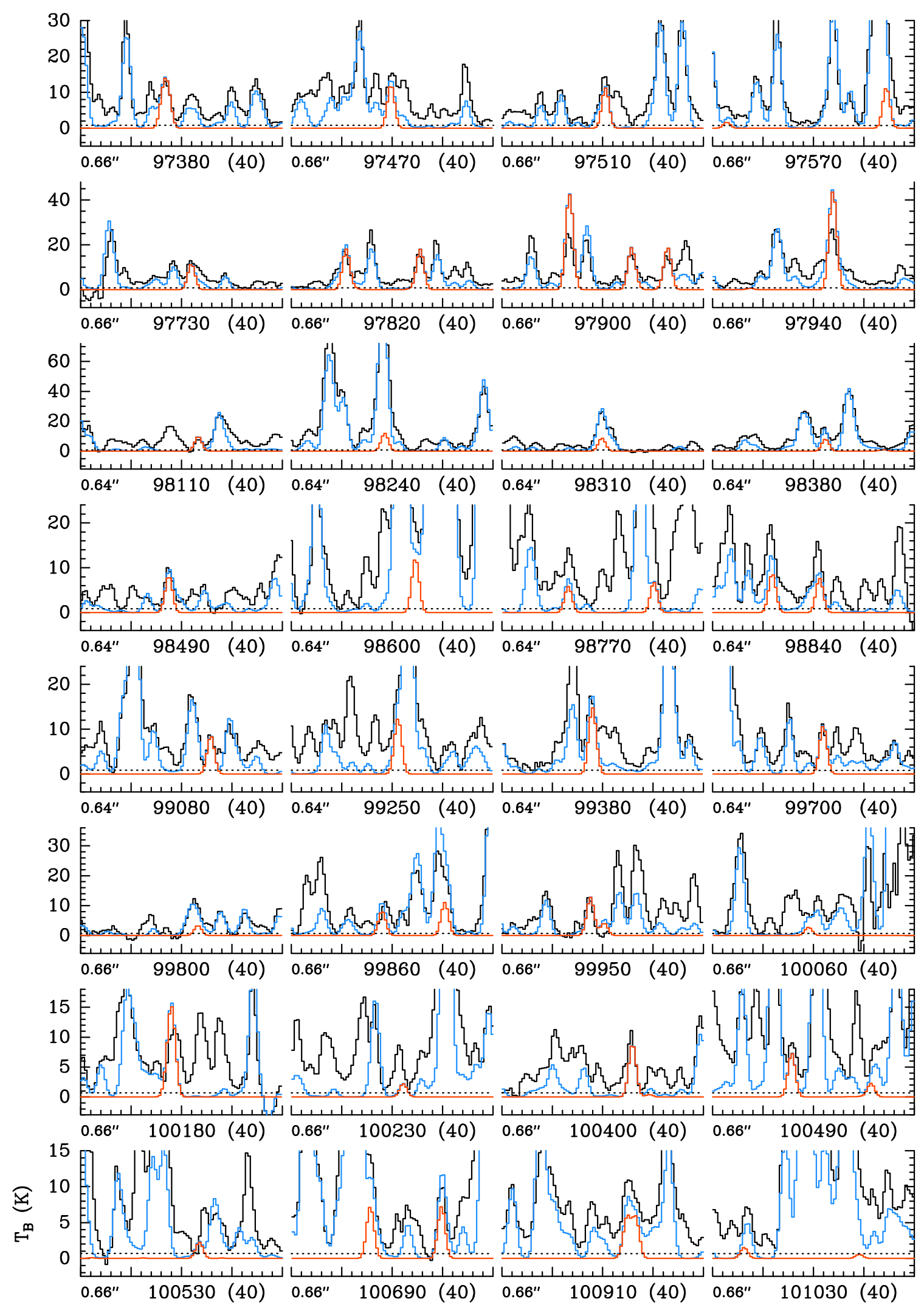

Fig. A.14. continued. 
A. Belloche et al.: Re-exploring molecular complexity with ALMA (ReMoCA): interstellar detection of urea
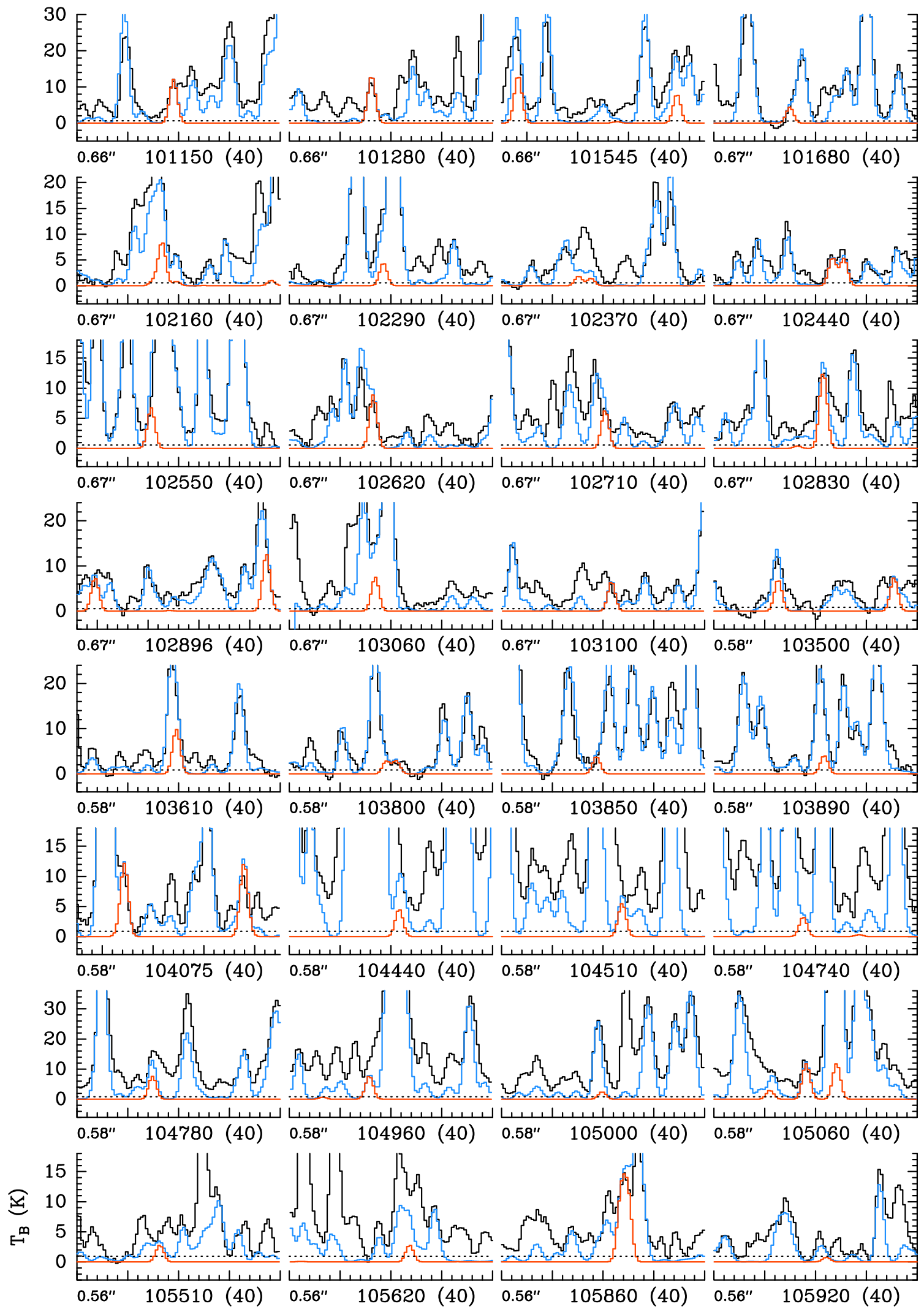

Fig. A.14. continued. 


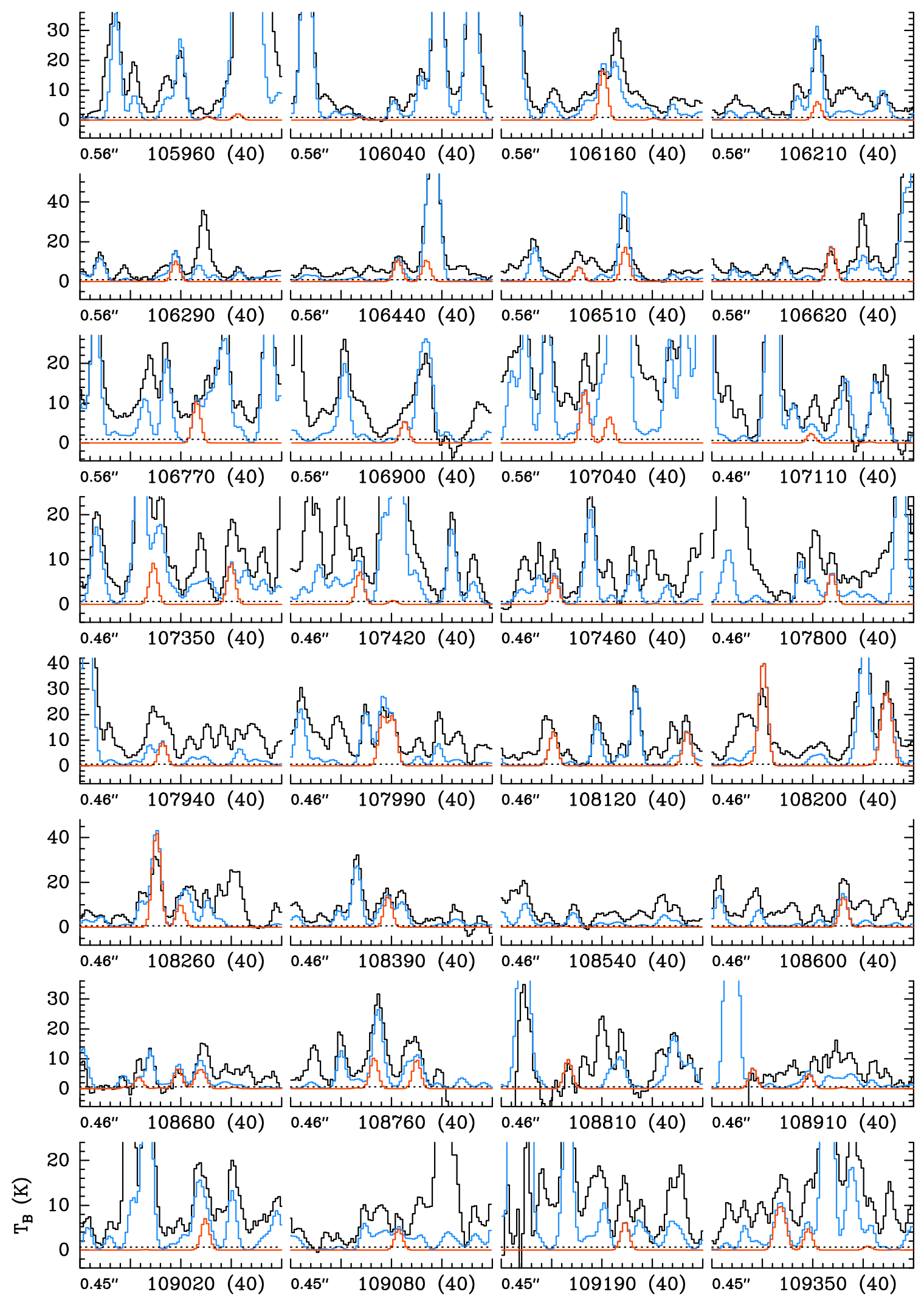

Fig. A.14. continued. 
A. Belloche et al.: Re-exploring molecular complexity with ALMA (ReMoCA): interstellar detection of urea
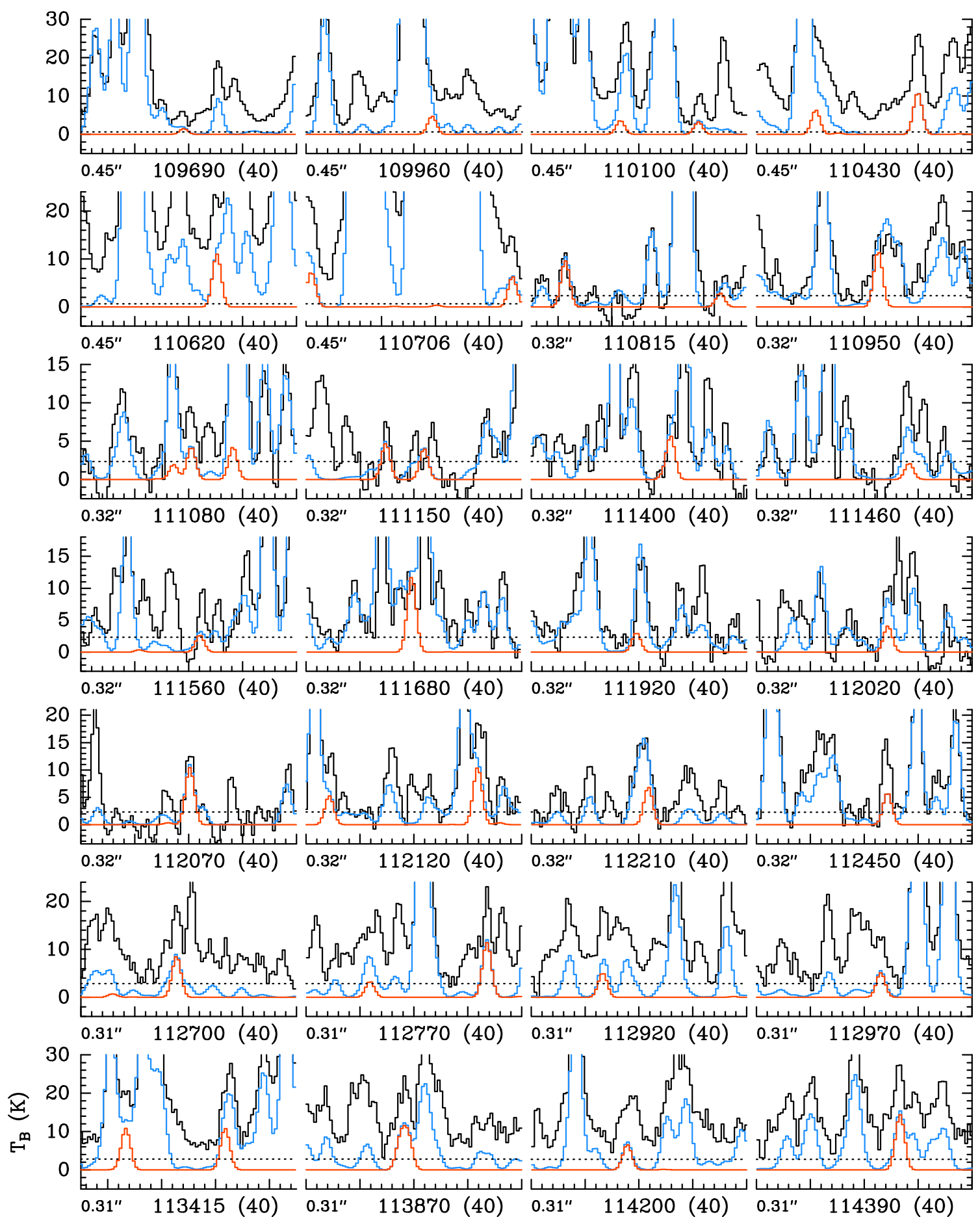

Fig. A.14. continued. 


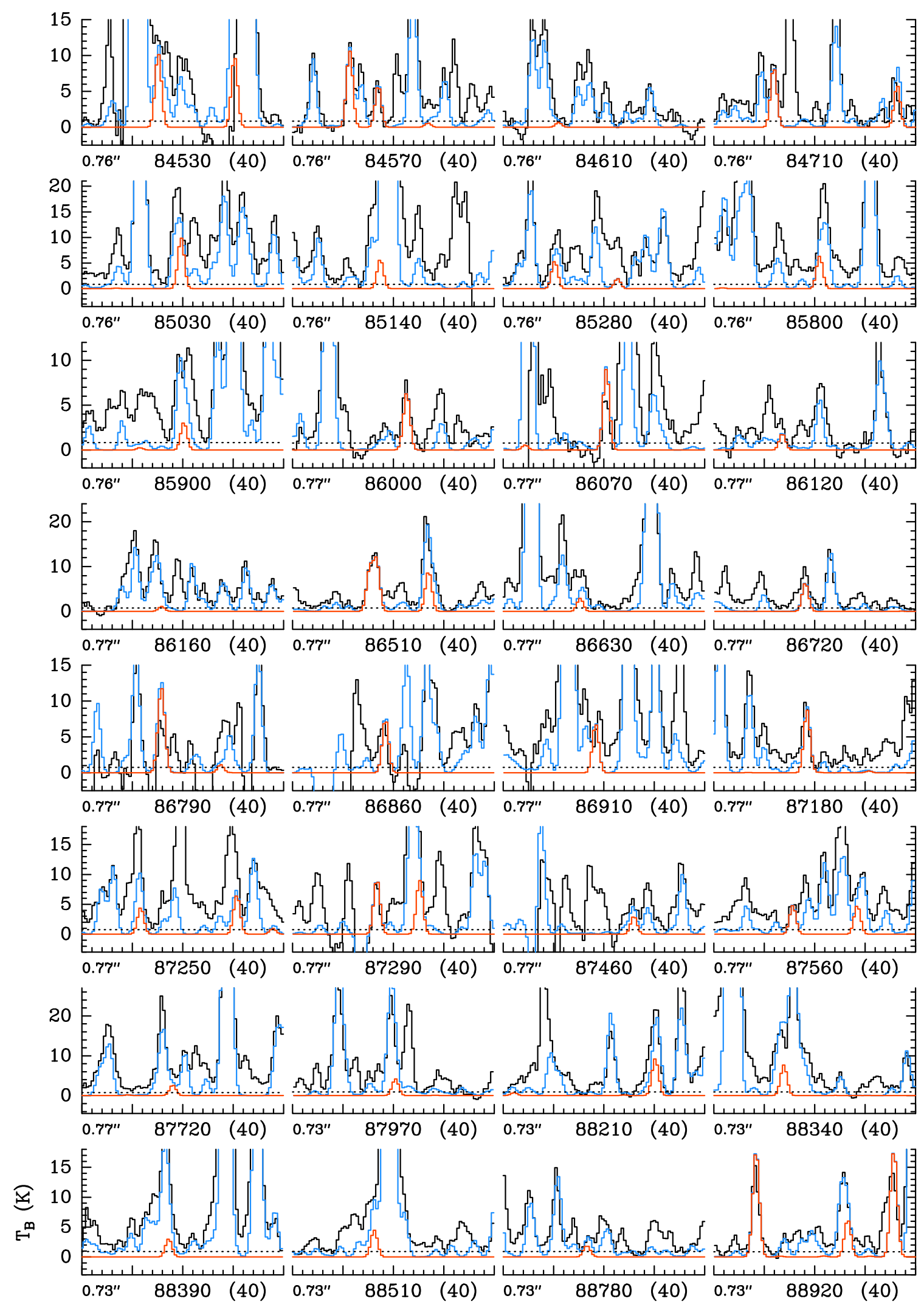

Fig. A.15. Same as Fig. A.1 for $\mathrm{CH}_{3} \mathrm{C}(\mathrm{O}) \mathrm{NH}_{2}, v_{\mathrm{t}}=1$. 
A. Belloche et al.: Re-exploring molecular complexity with ALMA (ReMoCA): interstellar detection of urea
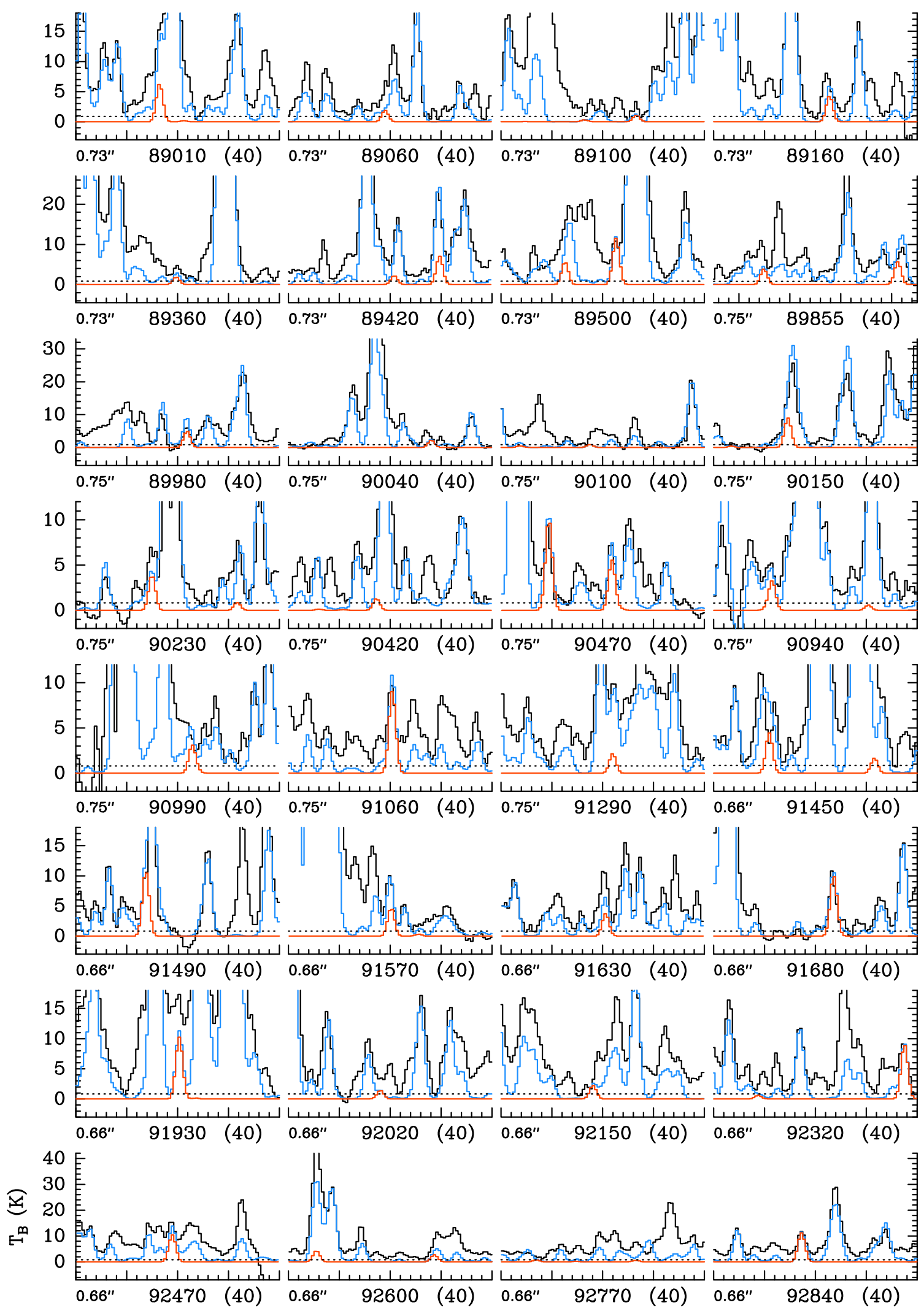

Fig. A.15. continued. 


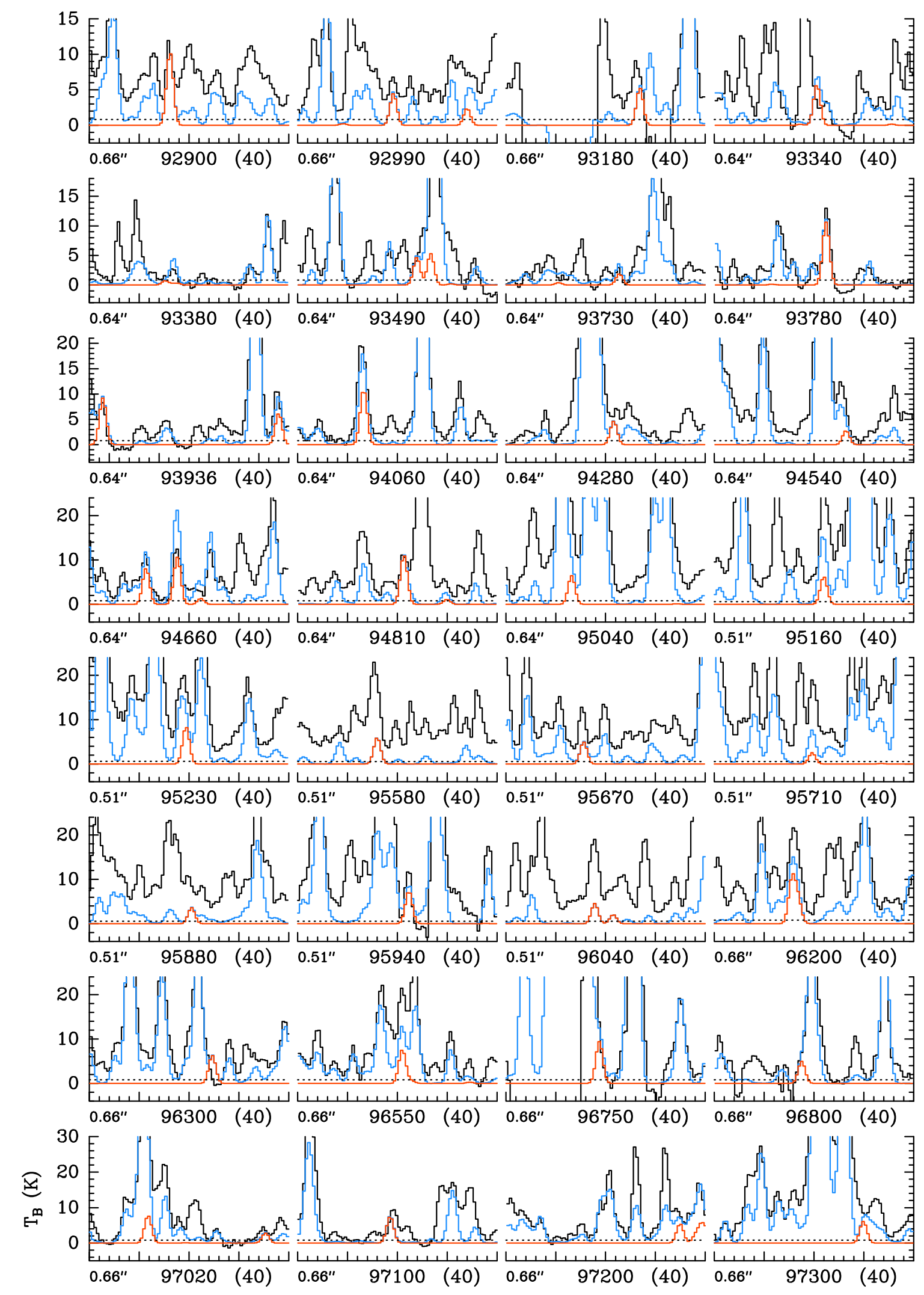

Fig. A.15. continued. 

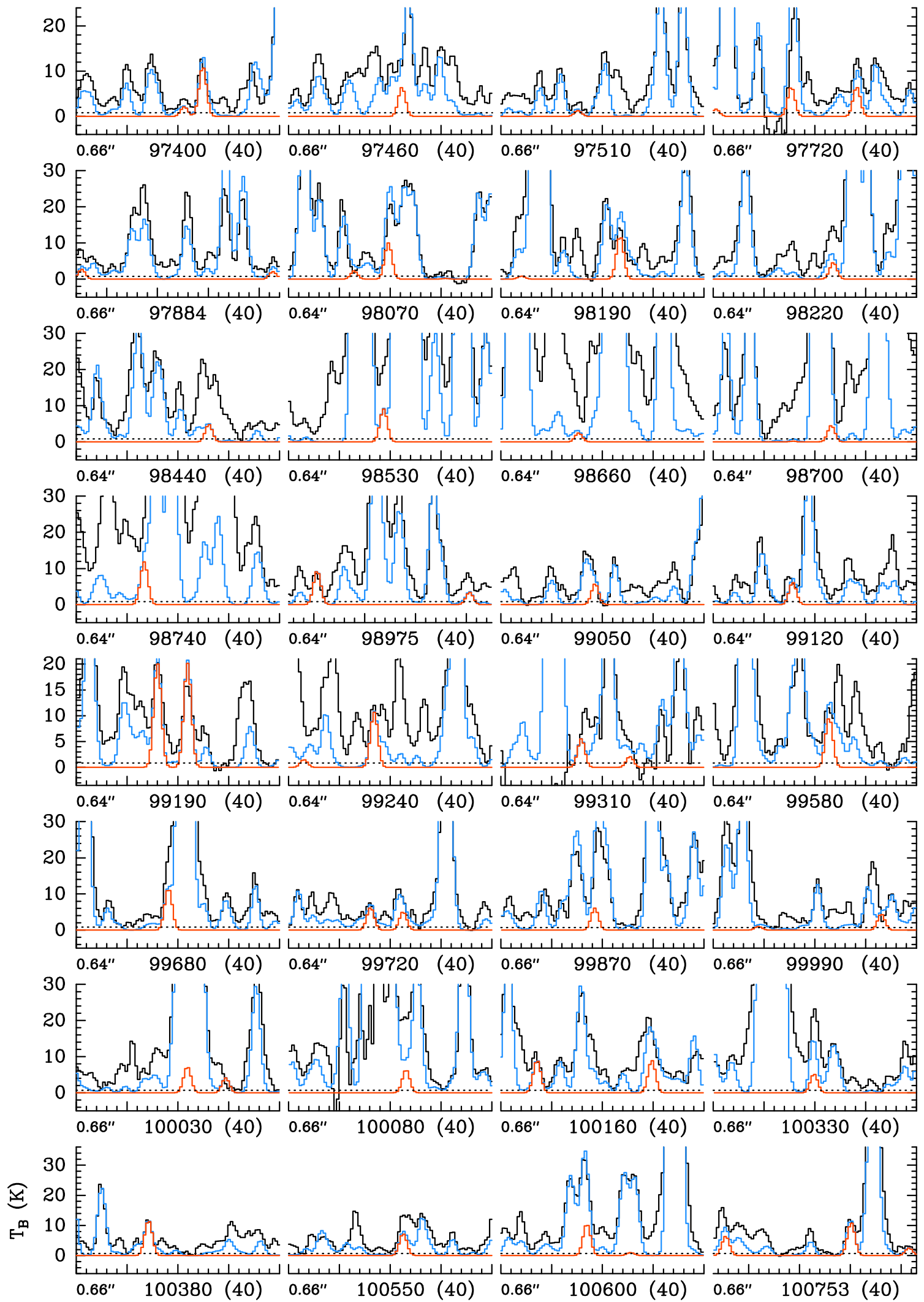

Fig. A.15. continued. 


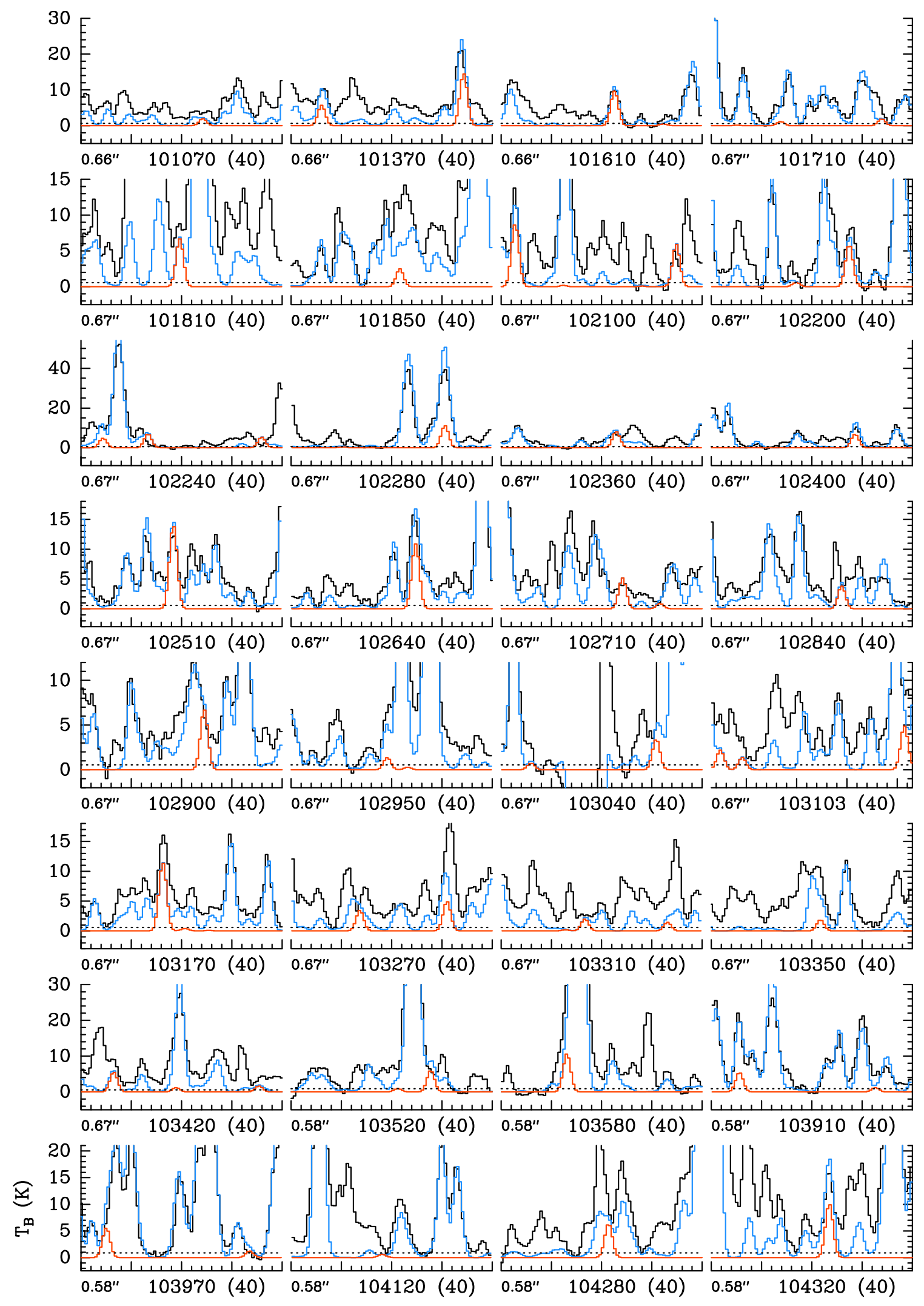

Fig. A.15. continued. 
A. Belloche et al.: Re-exploring molecular complexity with ALMA (ReMoCA): interstellar detection of urea
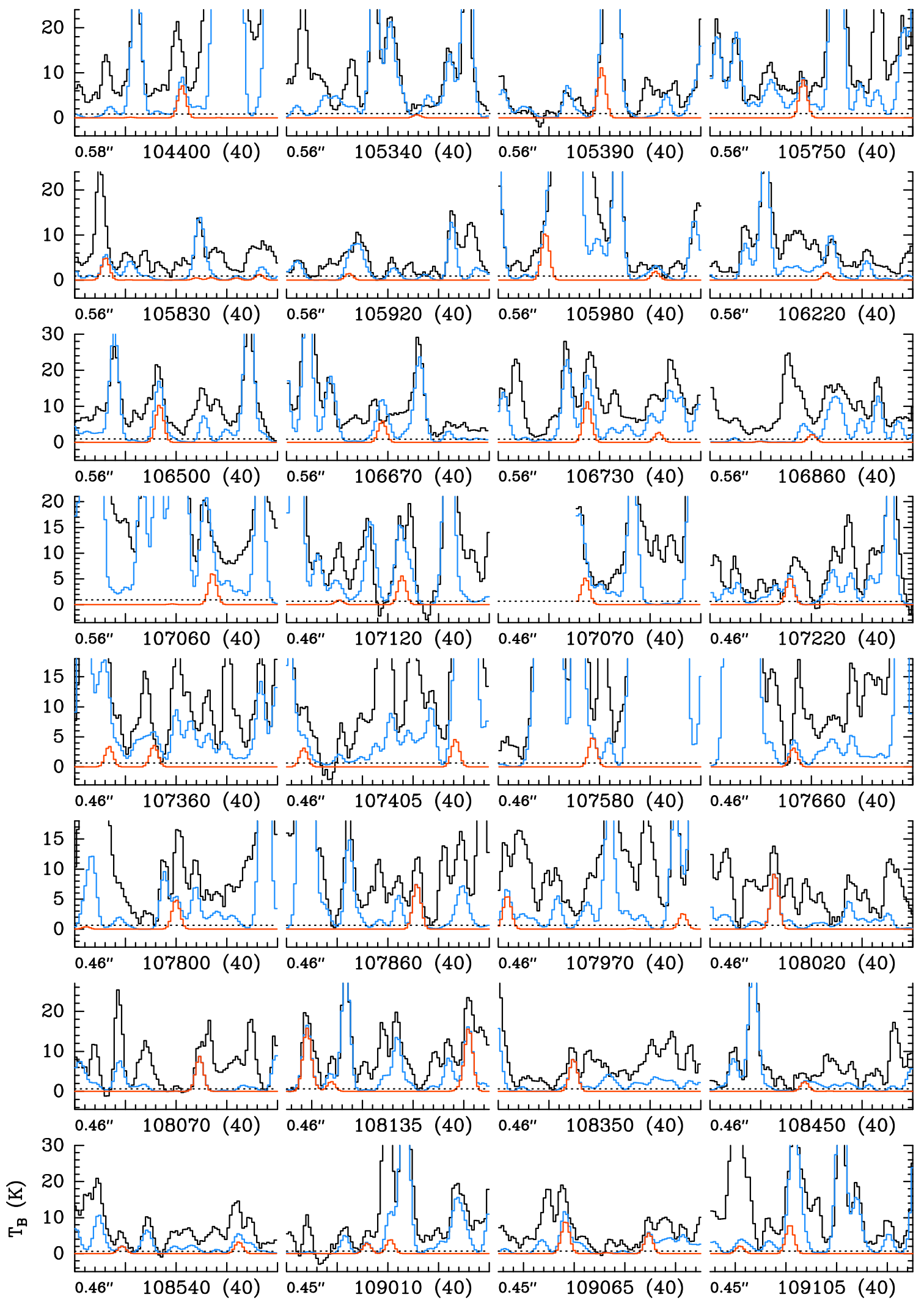

Fig. A.15. continued. 


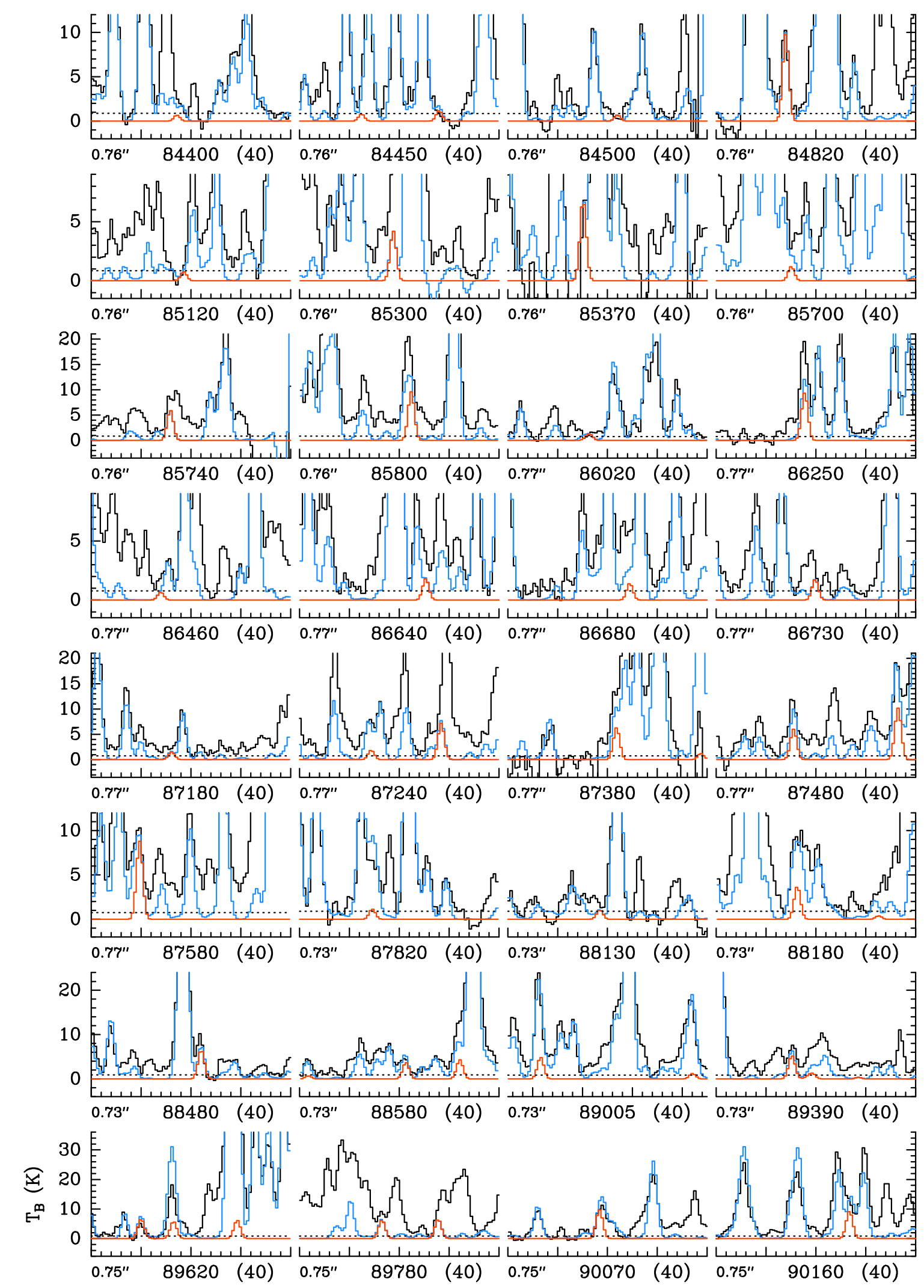

Fig. A.16. Same as Fig. A.1 for $\mathrm{CH}_{3} \mathrm{C}(\mathrm{O}) \mathrm{NH}_{2}, v_{\mathrm{t}}=2$. 
A. Belloche et al.: Re-exploring molecular complexity with ALMA (ReMoCA): interstellar detection of urea
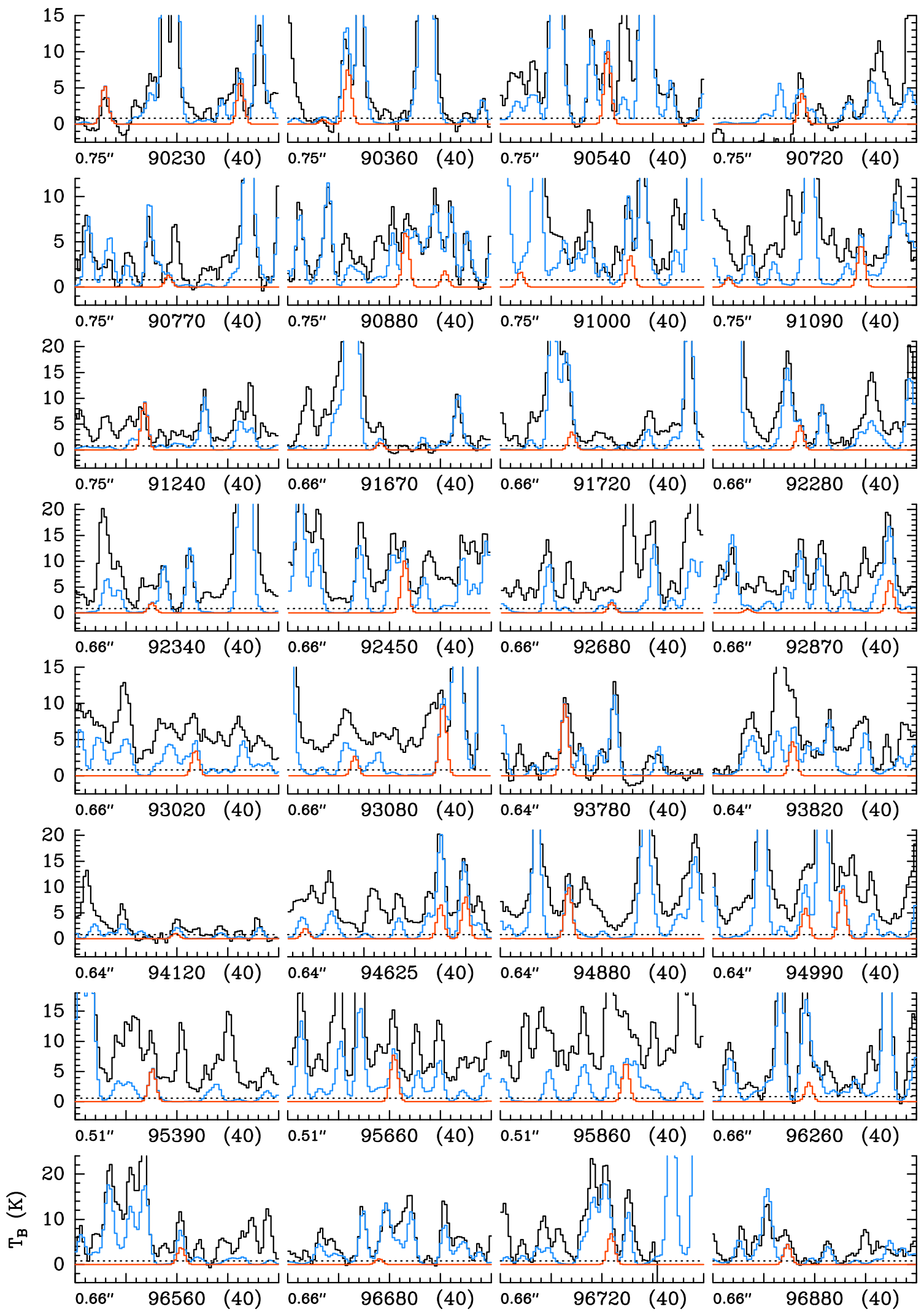

Fig. A.16. continued. 


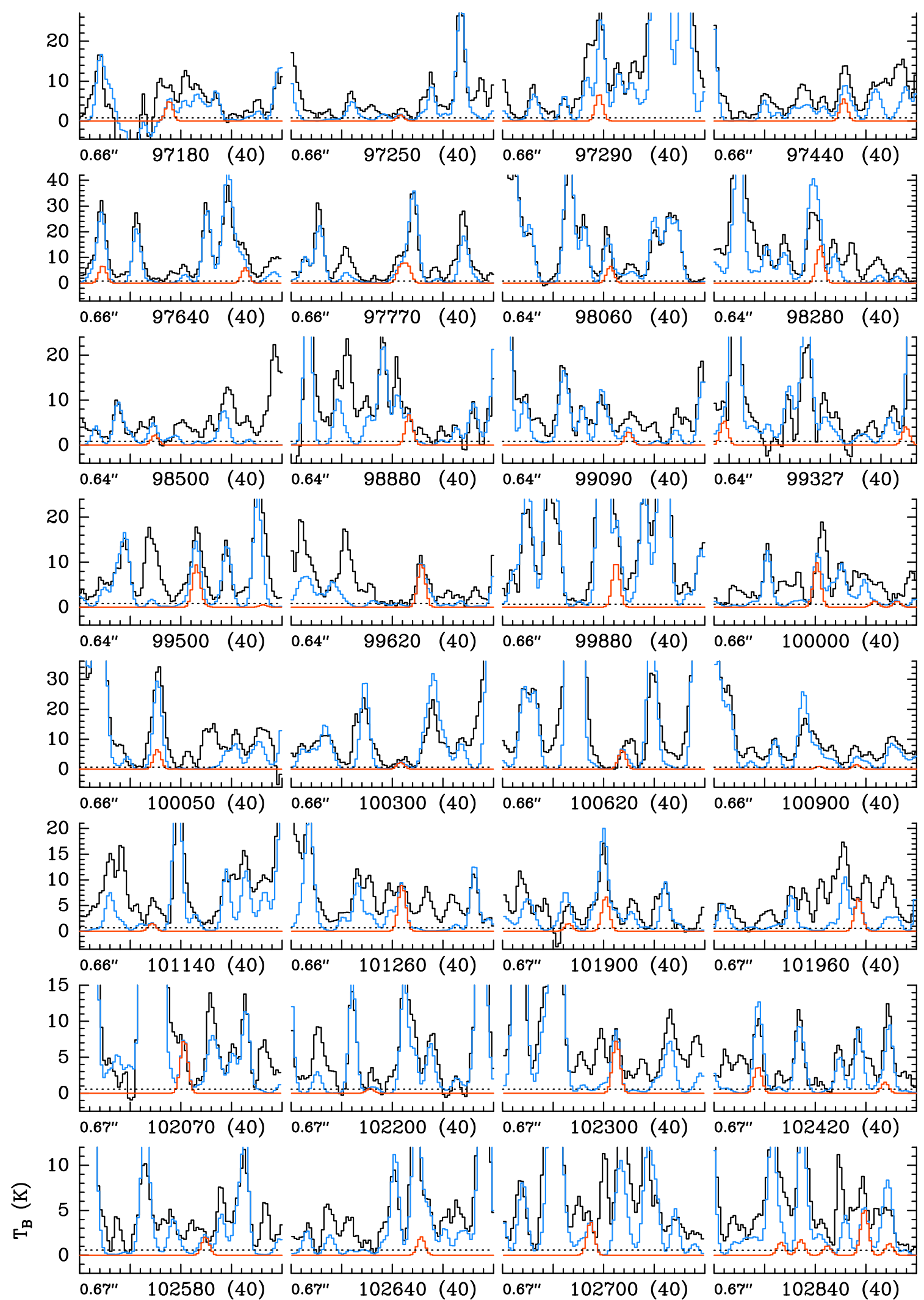

Fig. A.16. continued. 
A. Belloche et al.: Re-exploring molecular complexity with ALMA (ReMoCA): interstellar detection of urea
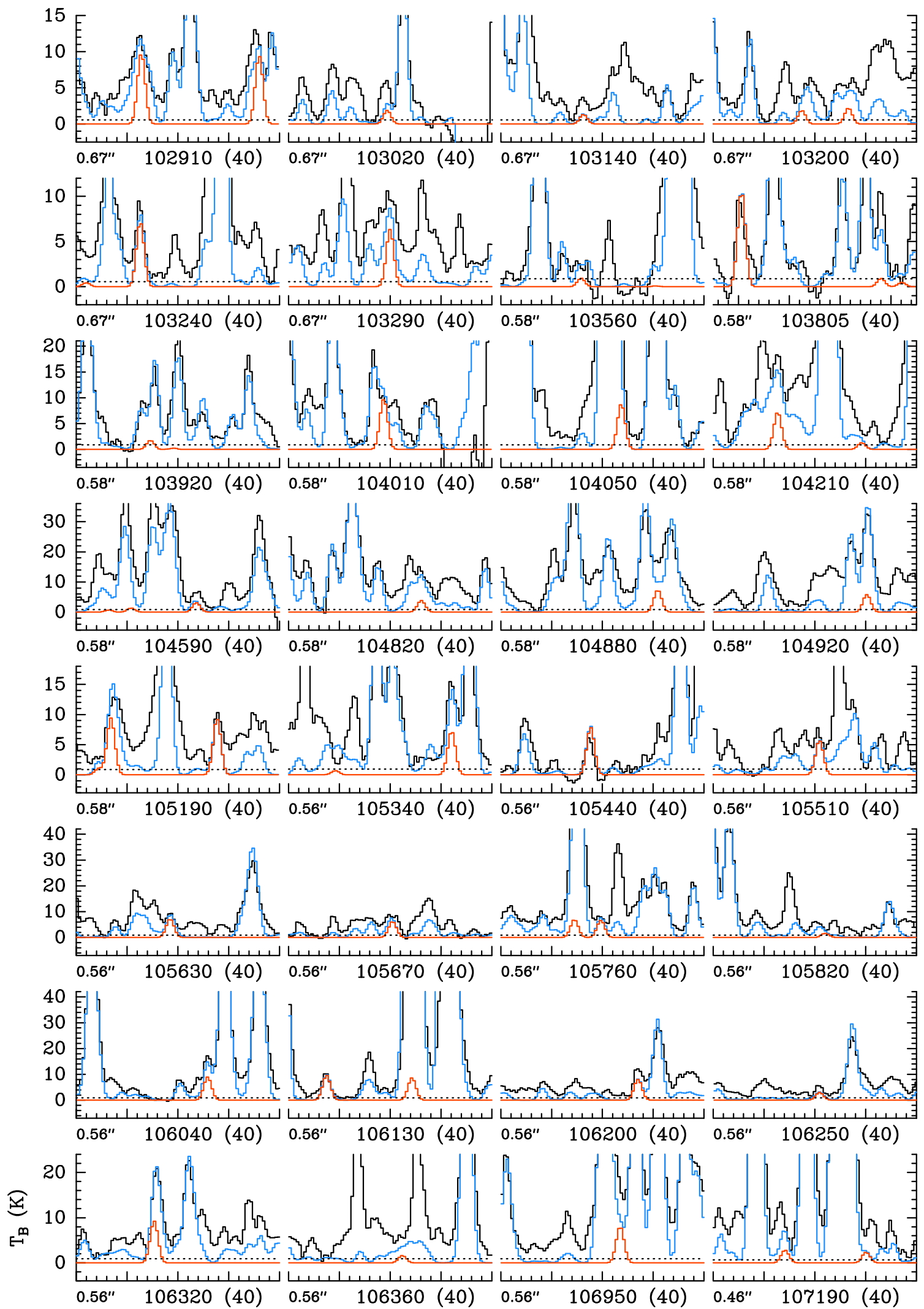

Fig. A.16. continued. 


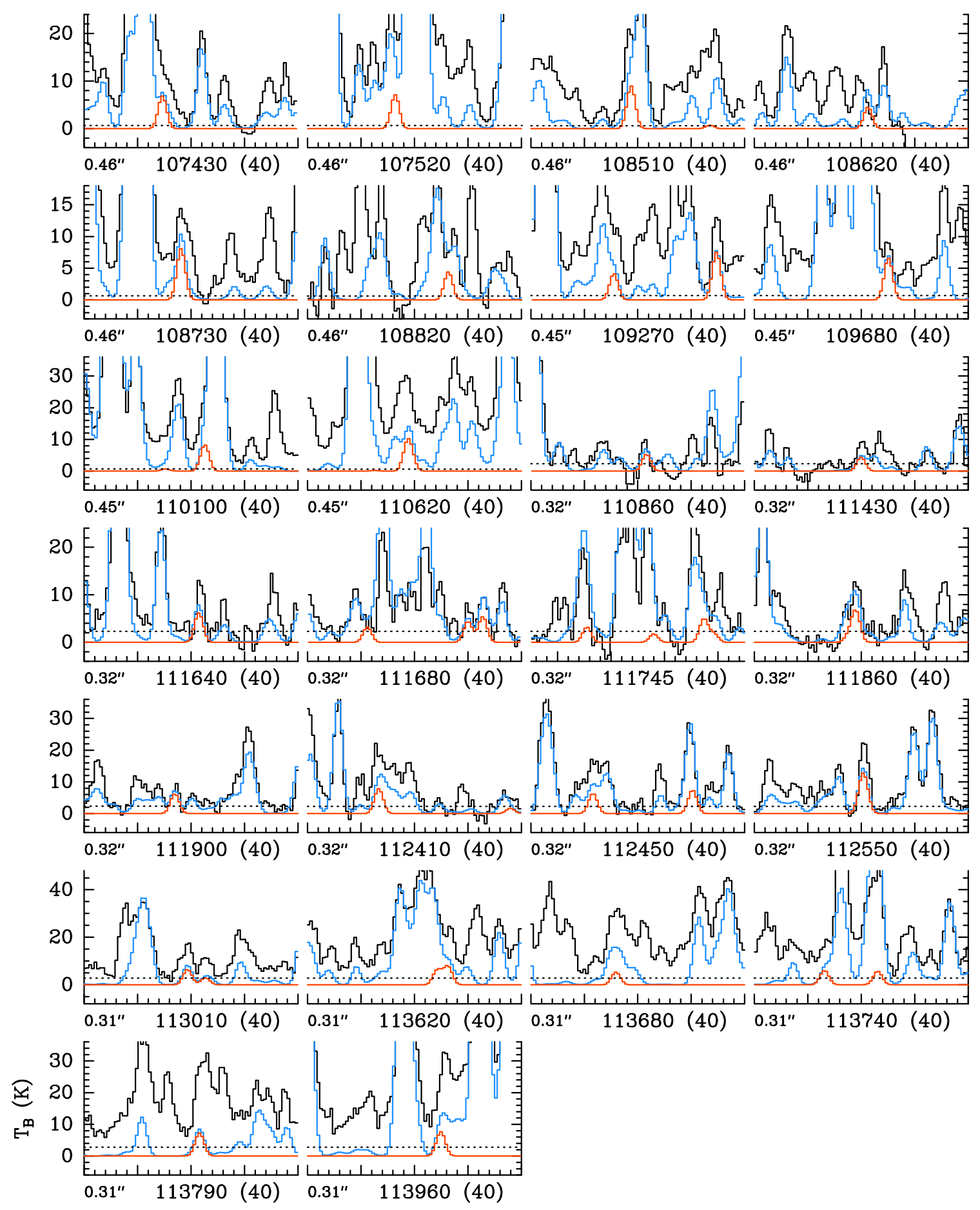

Fig. A.16. continued. 
A. Belloche et al.: Re-exploring molecular complexity with ALMA (ReMoCA): interstellar detection of urea
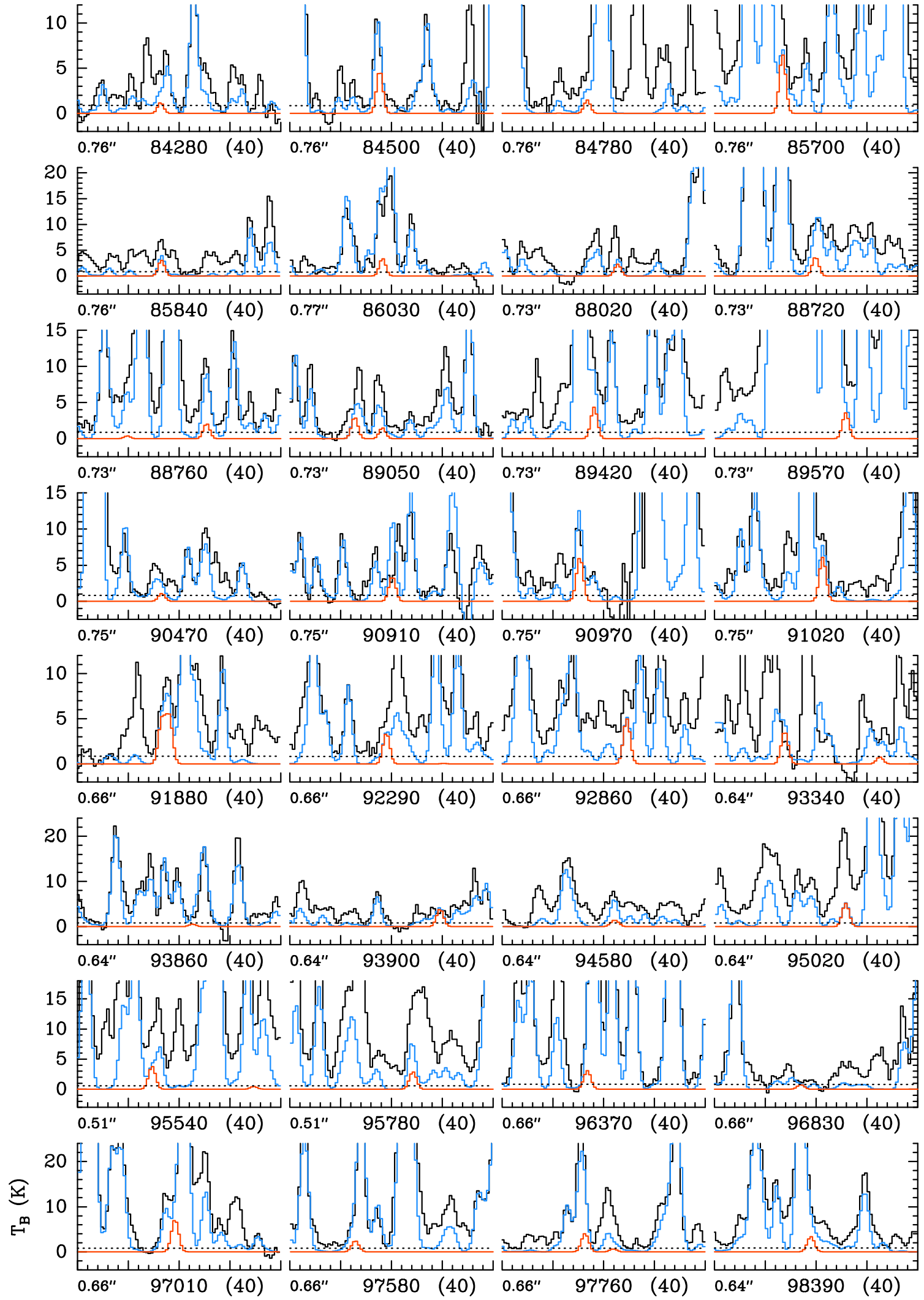

Fig. A.17. Same as Fig. A.1 for $\mathrm{CH}_{3} \mathrm{C}(\mathrm{O}) \mathrm{NH}_{2}, \Delta v_{\mathrm{t}} \neq 0$. 


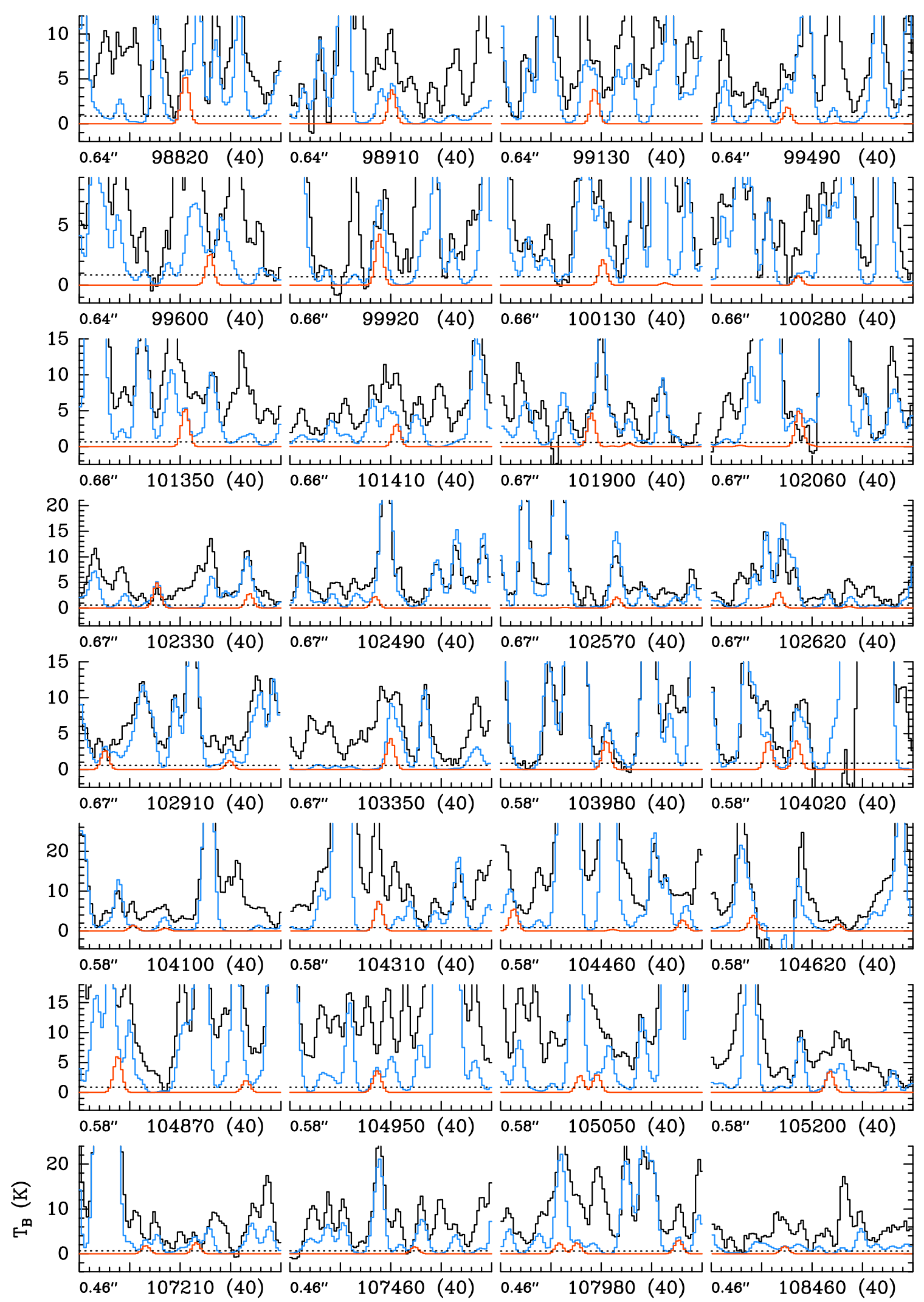

Fig. A.17. continued. 

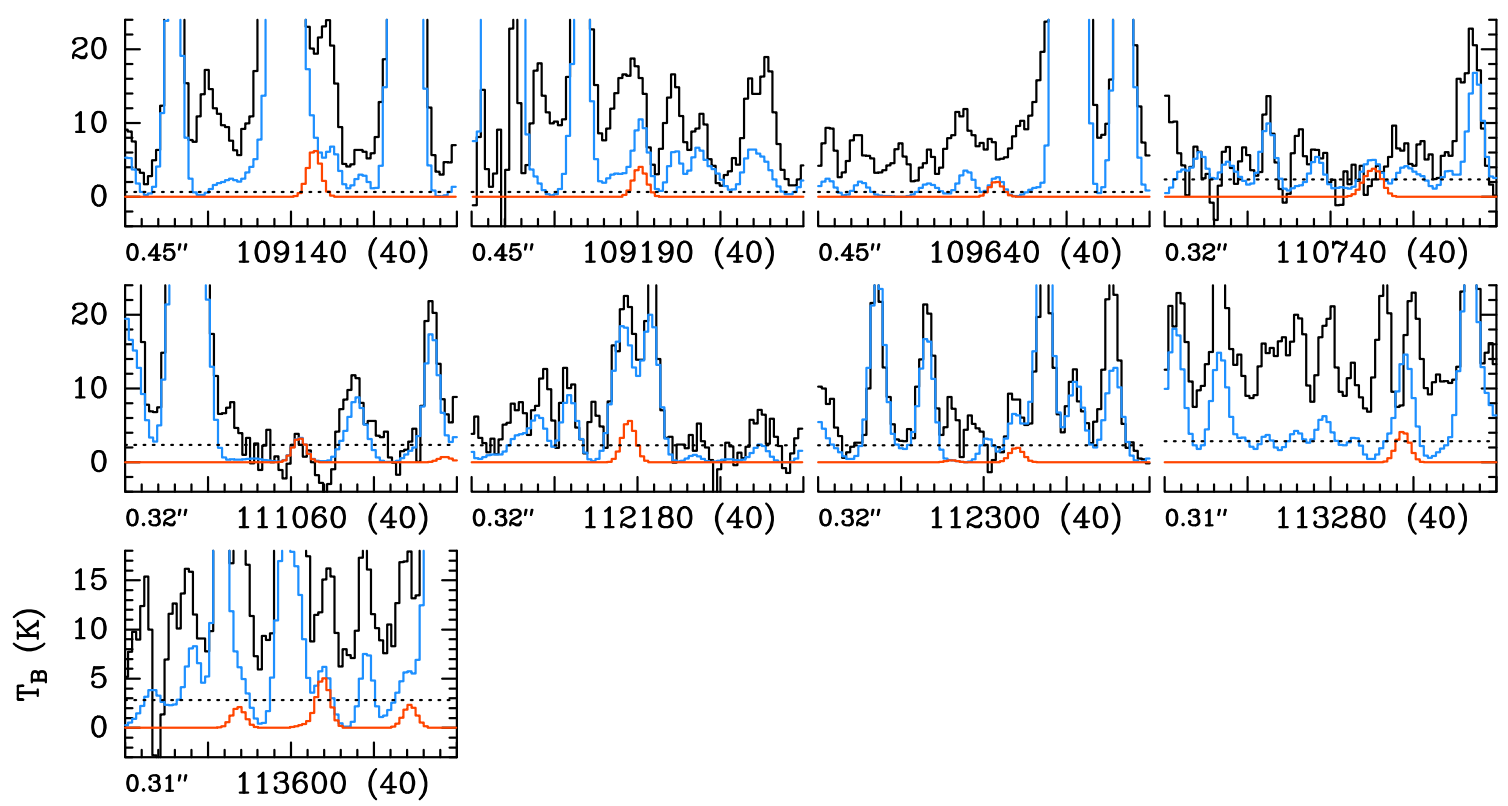

Fig. A.17. continued.

\section{Appendix B: Complementary figures: population diagrams}

Figures B.1-B.8 show the population diagrams of $\mathrm{NH}_{2} \mathrm{C}(\mathrm{O}) \mathrm{NH}_{2}, \mathrm{CH}_{3} \mathrm{NHCHO}, \mathrm{CH}_{3} \mathrm{NCO}, \mathrm{NH}_{2} \mathrm{CHO}$ and its ${ }^{13} \mathrm{C}$, ${ }^{18} \mathrm{O}$, and ${ }^{15} \mathrm{~N}$ isotopologs, and $\mathrm{CH}_{3} \mathrm{C}(\mathrm{O}) \mathrm{NH}_{2}$ toward Sgr B2(N1S).
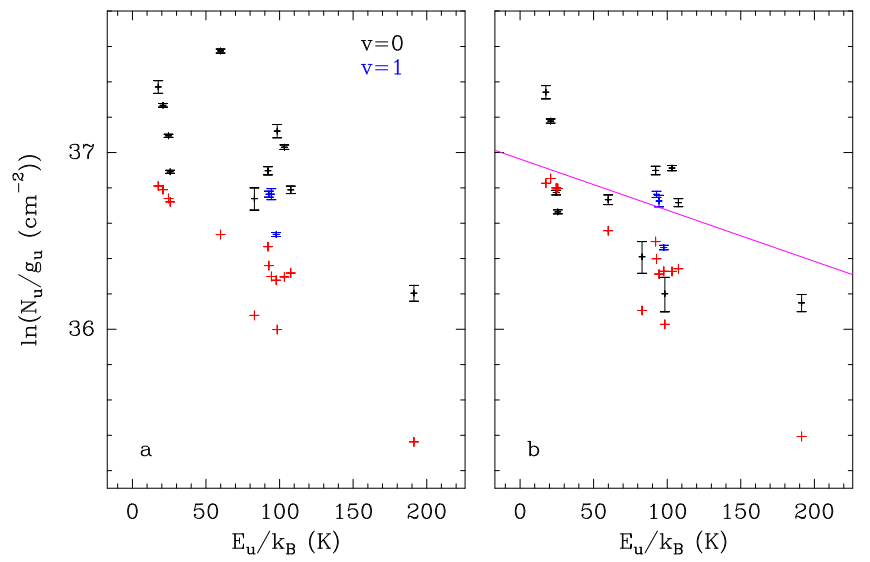

Fig. B.1. Population diagram of $\mathrm{NH}_{2} \mathrm{C}(\mathrm{O}) \mathrm{NH}_{2}$ toward $\mathrm{Sgr} \mathrm{B} 2(\mathrm{~N} 1 \mathrm{~S})$. The observed datapoints are shown in various colors (but not red) as indicated in the upper right corner of panel $a$ while the synthetic populations are shown in red. No correction is applied in panel $a$. In panel $b$, the optical depth correction has been applied to both the observed and synthetic populations and the contamination by all other species included in the full model has been removed from the observed datapoints. The purple line is a linear fit to the observed populations (in linear-logarithmic space).
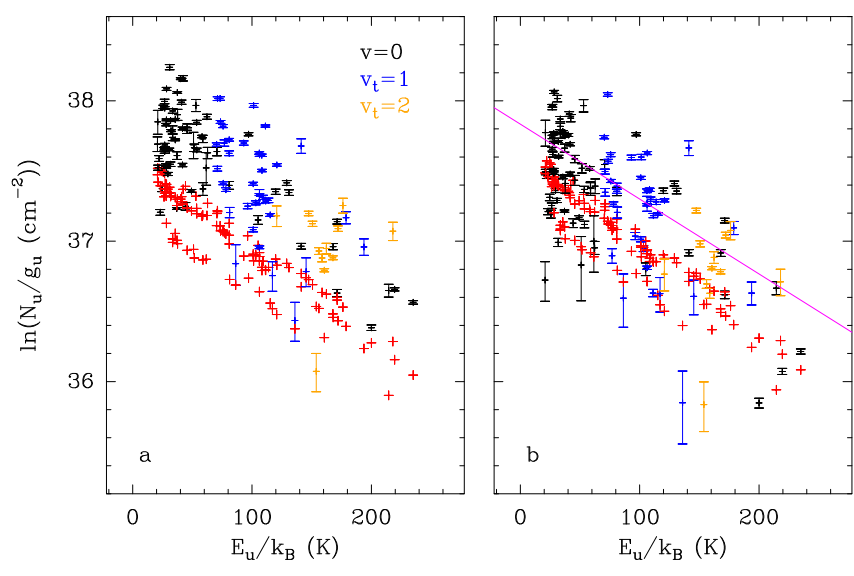

Fig. B.2. Same as Fig. B.1 for $\mathrm{CH}_{3} \mathrm{NHCHO}$
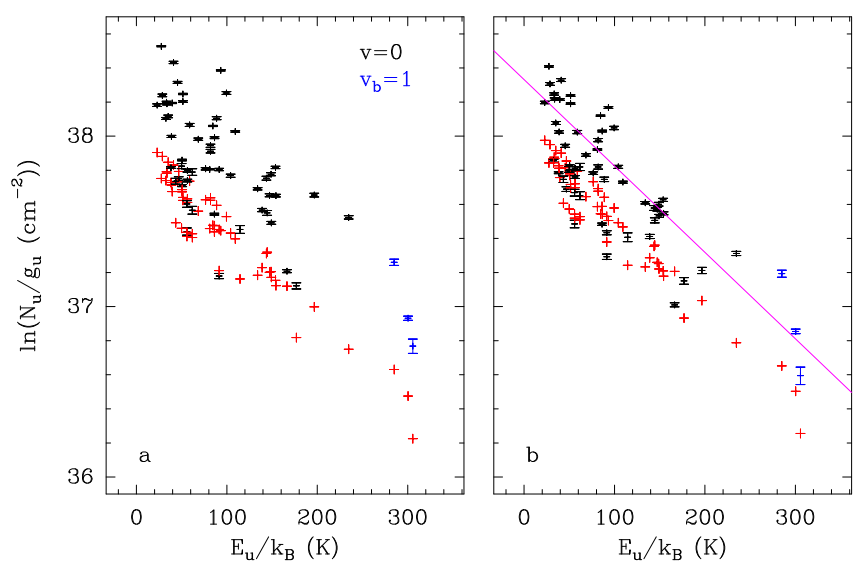

Fig. B.3. Same as Fig. B.1 for $\mathrm{CH}_{3} \mathrm{NCO}$. 
A\&A 628, A10 (2019)

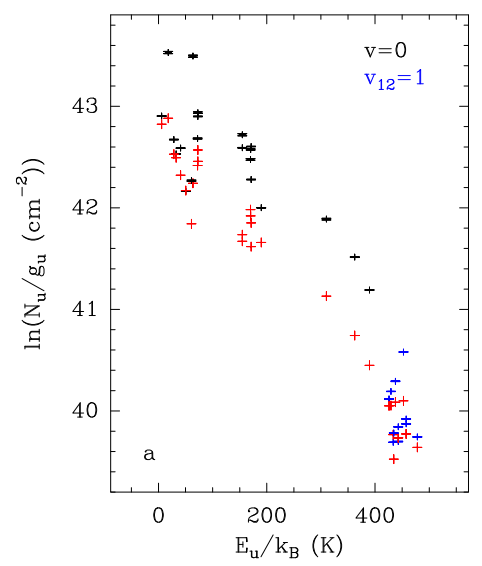

Fig. B.4. Same as Fig. B.1 for $\mathrm{NH}_{2} \mathrm{CHO}$.
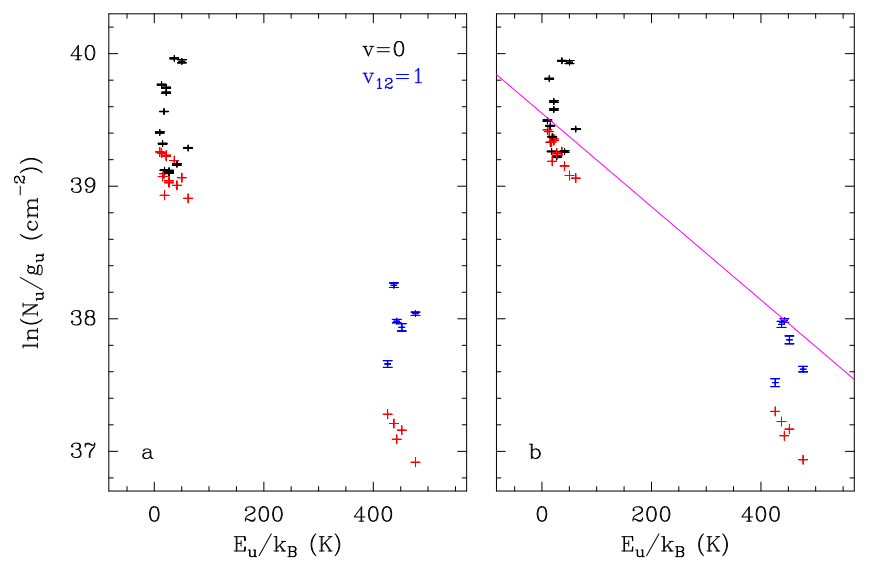

Fig. B.5. Same as Fig. B.1 for $\mathrm{NH}_{2}{ }^{13} \mathrm{CHO}$.

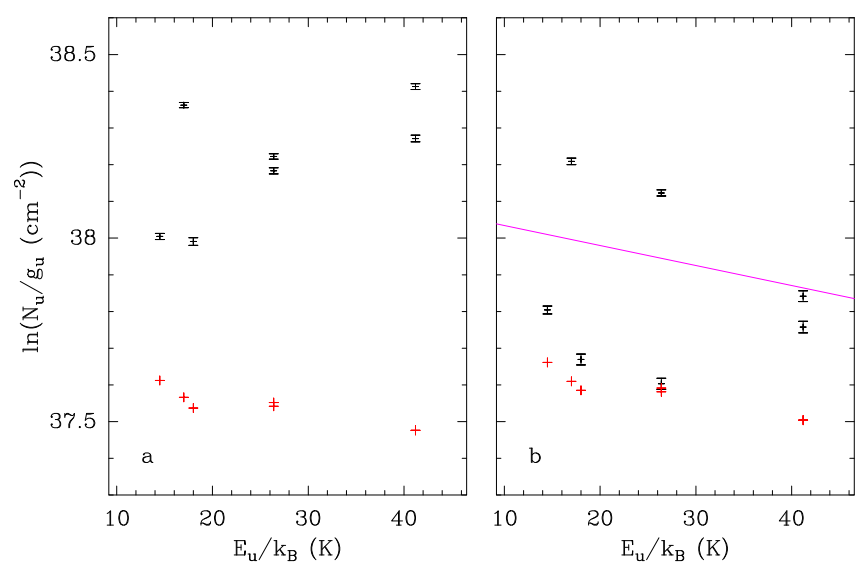

Fig. B.6. Same as Fig. B.1 for $\mathrm{NH}_{2} \mathrm{CH}^{18} \mathrm{O}$.
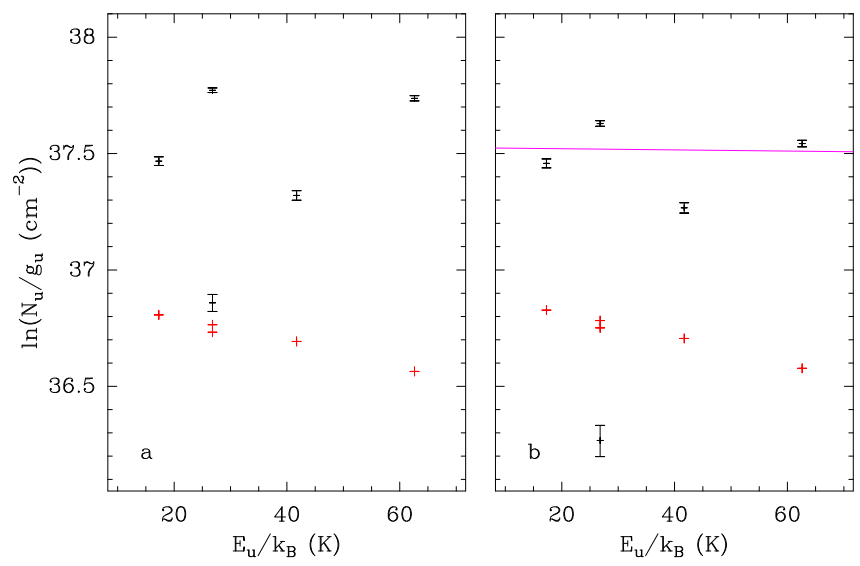

Fig. B.7. Same as Fig. B.1 for ${ }^{15} \mathrm{NH}_{2} \mathrm{CHO}$.
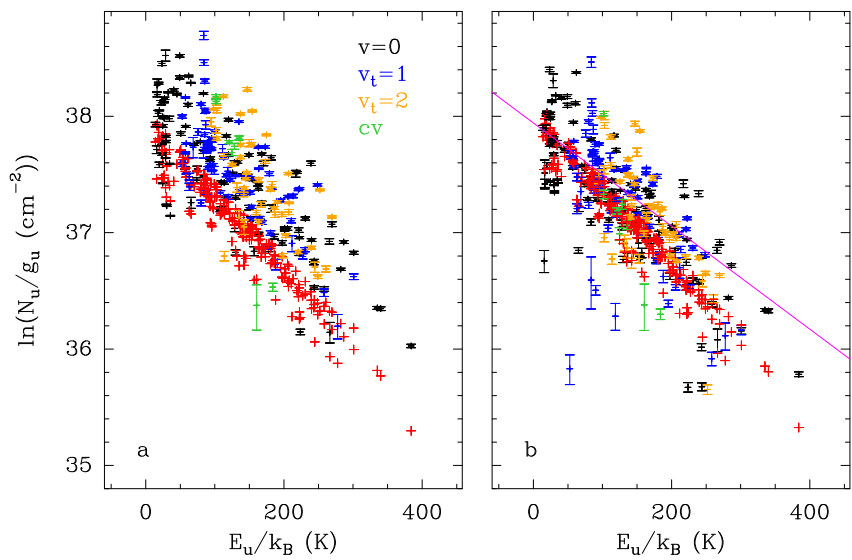

Fig. B.8. Same as Fig. B.1 for $\mathrm{CH}_{3} \mathrm{C}(\mathrm{O}) \mathrm{NH}_{2}$. 